\author{
Supporting Information
}

for

\title{
A straightforward metal-free synthesis of 2-substituted thiazolines in air
}

Michael Trose, Faïma Lazreg, Mathieu Lesieur and Catherine S. J. Cazin*

School of Chemistry University of St Andrews, St Andrews, KY16 9ST, United Kingdom Fax: (+) 44 (0) 1334 463808; E-mail: cc111@st-andrews.ac.uk 


\section{Table of Contents}

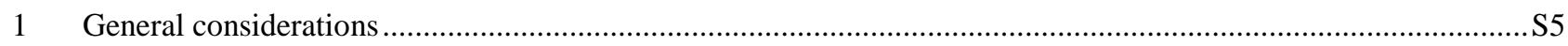

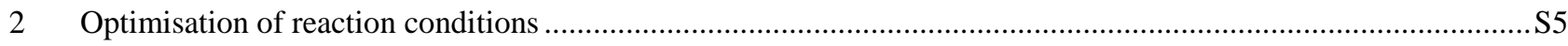

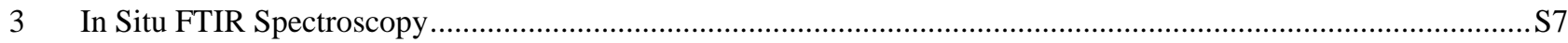

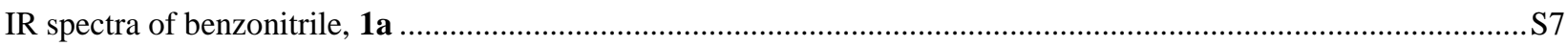

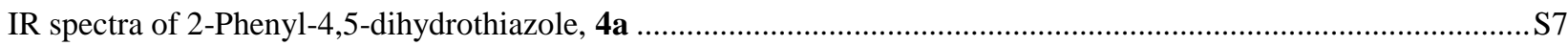

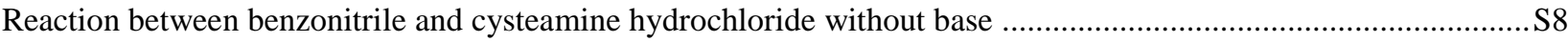

Reaction between benzonitrile and cysteamine hydrochloride in the presence of $\mathrm{NaOH}$....................................S10

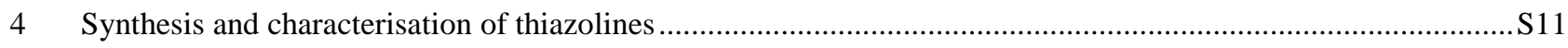

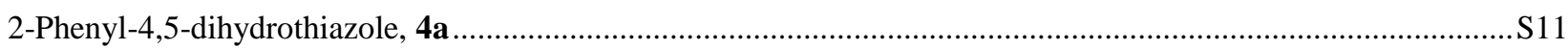

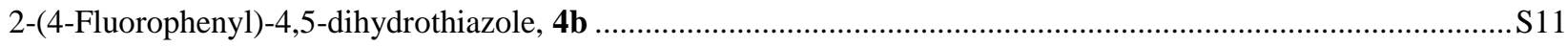

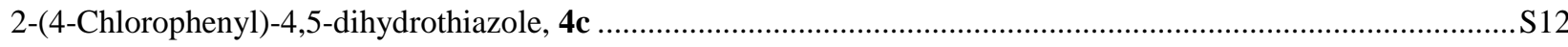

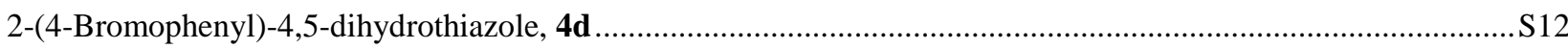

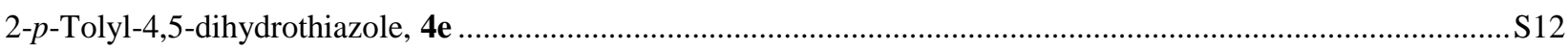

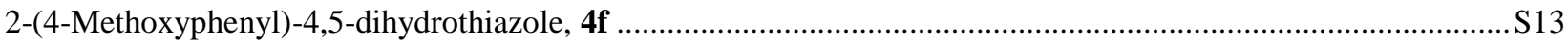

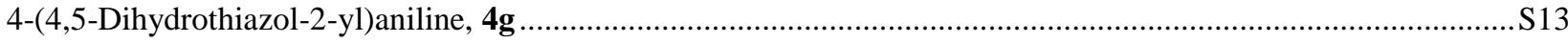

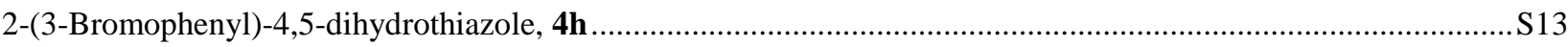

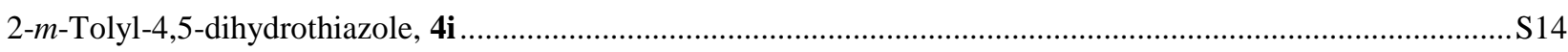

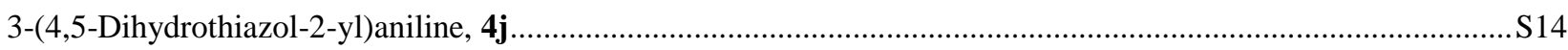

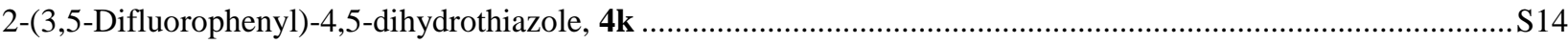

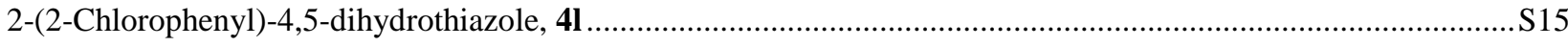

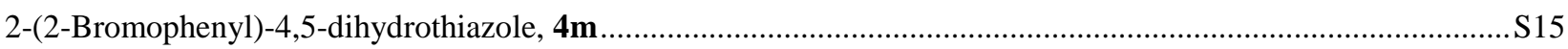

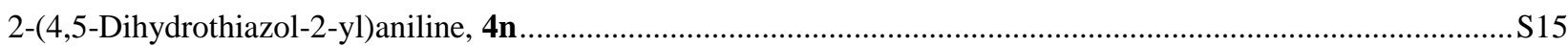

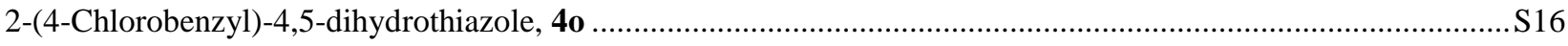

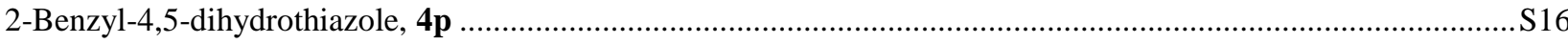

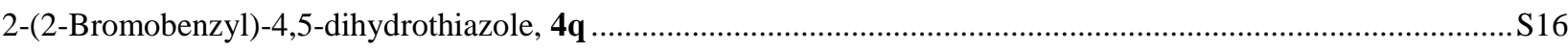

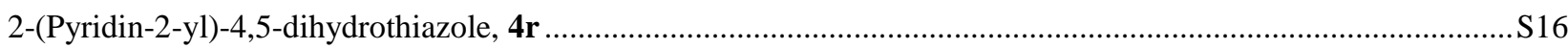

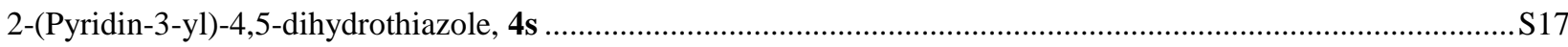

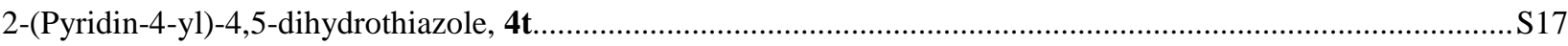

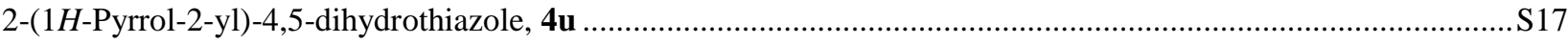

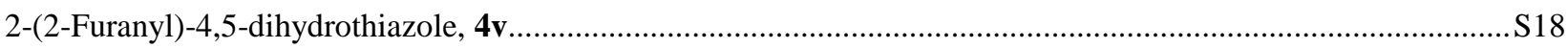

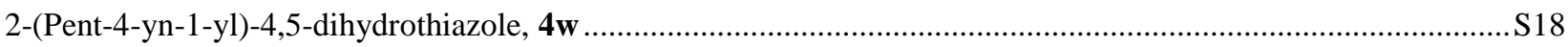

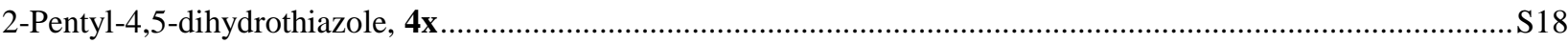

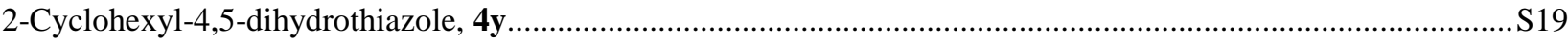

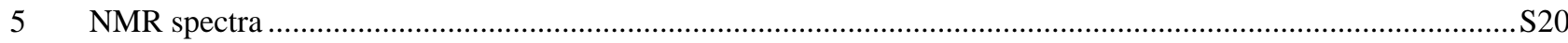

${ }^{1} \mathrm{H}$ NMR (500 MHz, $\mathrm{CDCl}_{3}, 298 \mathrm{~K}$, TMS) of 2-phenyl-4,5-dihydrothiazole, 4a .............................................S20

${ }^{13} \mathrm{C}-\left\{{ }^{1} \mathrm{H}\right\}$ NMR $\left(125.7 \mathrm{MHz}, \mathrm{CDCl}_{3}, 298 \mathrm{~K}\right)$ of 2-phenyl-4,5-dihydrothiazole, 4a ...........................................S20

${ }^{1} \mathrm{H}$ NMR (400 MHz, $\left.\mathrm{CDCl}_{3}, 298 \mathrm{~K}, \mathrm{TMS}\right)$ of 2-(4-fluorophenyl)-4,5-dihydrothiazole, 4b...................................... 
${ }^{13} \mathrm{C}-\left\{{ }^{1} \mathrm{H}\right\}$ NMR (75 MHz, $\left.\mathrm{CDCl}_{3}, 298 \mathrm{~K}\right)$ of 2-(4-fluorophenyl)-4,5-dihydrothiazole, $\mathbf{4 b}$. S21

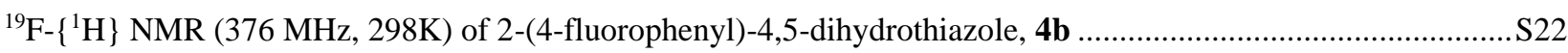

${ }^{1} \mathrm{H}$ NMR (400 MHz, CDCl3, 298K, TMS) of 2-(4-chlorophenyl)-4,5-dihydrothiazole, 4c...................................S23

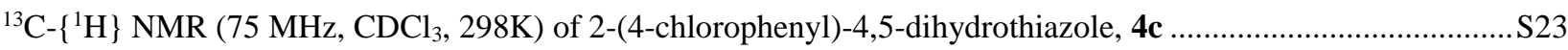

${ }^{1} \mathrm{H}$ NMR (400 MHz, $\left.\mathrm{CDCl}_{3}, 298 \mathrm{~K}, \mathrm{TMS}\right)$ of 2-(4-bromophenyl)-4,5-dihydrothiazole, 4d ..................................S24

${ }^{13} \mathrm{C}-\left\{{ }^{1} \mathrm{H}\right\}$ NMR $\left(75 \mathrm{MHz}, \mathrm{CDCl}_{3}, 298 \mathrm{~K}\right)$ of 2-(4-bromophenyl)-4,5-dihydrothiazole, 4d.....................................S24

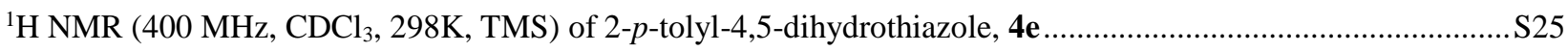

${ }^{13} \mathrm{C}-\left\{{ }^{1} \mathrm{H}\right\}$ NMR $\left(75 \mathrm{MHz}, \mathrm{CDCl}_{3}, 298 \mathrm{~K}\right)$ of 2-p-tolyl-4,5-dihydrothiazole, 4e................................................S25

${ }^{1} \mathrm{H}$ NMR (500 MHz, $\left.\mathrm{CDCl}_{3}, 298 \mathrm{~K}, \mathrm{TMS}\right)$ of 2-(4-methoxyphenyl)-4,5-dihydrothiazole, $\mathbf{4 f}$.............................S26

${ }^{13} \mathrm{C}-\left\{{ }^{1} \mathrm{H}\right\} \mathrm{NMR}\left(125.7 \mathrm{MHz}, \mathrm{CDCl}_{3}, 298 \mathrm{~K}\right)$ of 2-(4-methoxyphenyl)-4,5-dihydrothiazole, $4 \mathbf{f}$.............................S26

${ }^{1} \mathrm{H}$ NMR (400 MHz, $\mathrm{CDCl}_{3}, 298 \mathrm{~K}$, TMS) of 4-(4,5-dihydrothiazol-2-yl)aniline, 4g .......................................S27

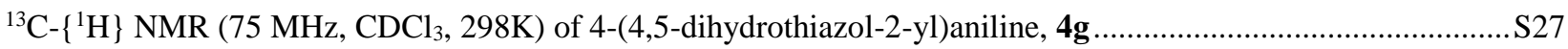

${ }^{1} \mathrm{H}$ NMR (400 MHz, $\left.\mathrm{CDCl}_{3}, 298 \mathrm{~K}, \mathrm{TMS}\right)$ of 2-(3-bromophenyl)-4,5-dihydrothiazole, 4h..................................S28

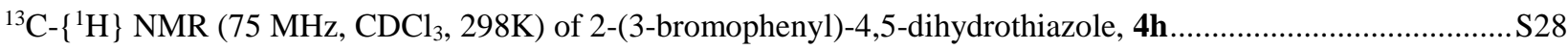

${ }^{1} \mathrm{H}$ NMR (500 MHz, $\mathrm{CDCl}_{3}, 298 \mathrm{~K}$, TMS) of 2- $m$-tolyl-4,5-dihydrothiazole, 4i................................................S29

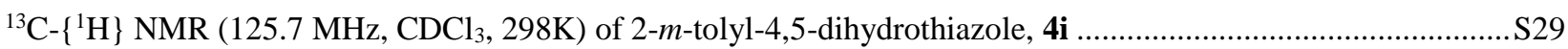

${ }^{1} \mathrm{H}$ NMR (400 MHz, $\mathrm{CDCl}_{3}$, 298K, TMS) of 3-(4,5-dihydrothiazol-2-yl)aniline, 4j j........................................S30

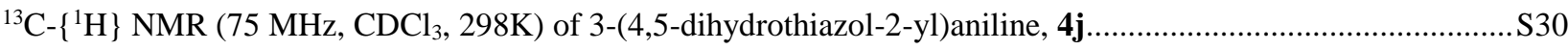

${ }^{1} \mathrm{H}$ NMR (400 MHz, $\mathrm{CDCl}_{3}, 298 \mathrm{~K}$, TMS) of 2-(3,5-difluorophenyl)-4,5-dihydrothiazole, 4k .............................S31

${ }^{13} \mathrm{C}-\left\{{ }^{1} \mathrm{H}\right\}$ NMR $\left(75 \mathrm{MHz}, \mathrm{CDCl}_{3}, 298 \mathrm{~K}\right)$ of 2-(3,5-difluorophenyl)-4,5-dihydrothiazole, 4k ...............................S31

${ }^{19} \mathrm{~F}-\left\{{ }^{1} \mathrm{H}\right\}$ NMR $(376 \mathrm{MHz}, 298 \mathrm{~K})$ of 2-(3,5-difluorophenyl)-4,5-dihydrothiazole, 4k .......................................S32

${ }^{1} \mathrm{H}$ NMR (500 MHz, $\left.\mathrm{CDCl}_{3}, 298 \mathrm{~K}, \mathrm{TMS}\right)$ of 2-(2-chlorophenyl)-4,5-dihydrothiazole, 41 ...................................S33

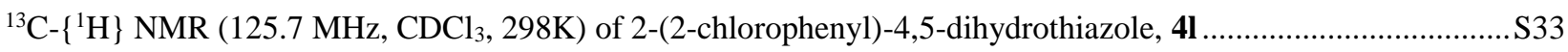

${ }^{1} \mathrm{H}$ NMR (400 MHz, $\left.\mathrm{CDCl}_{3}, 298 \mathrm{~K}, \mathrm{TMS}\right)$ of 2-(2-bromophenyl)-4,5-dihydrothiazole, 4m..................................S34

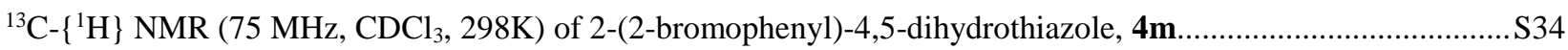

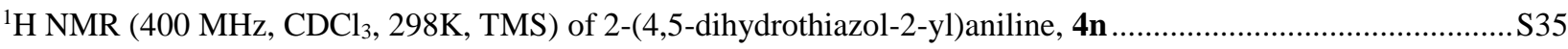

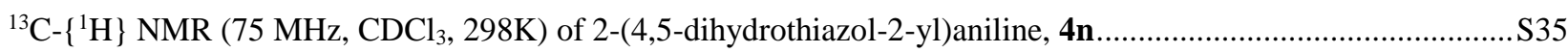

${ }^{1} \mathrm{H}$ NMR (400 MHz, $\mathrm{CDCl}_{3}, 298 \mathrm{~K}$, TMS) of 2-(4-chlorobenzyl)-4,5-dihydrothiazole, 4o.....................................S36

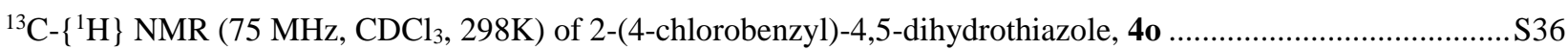

${ }^{1} \mathrm{H}$ NMR (400 MHz, $\mathrm{CDCl}_{3}, 298 \mathrm{~K}$, TMS) of 2-benzyl-4,5-dihydrothiazole, 4p ...............................................S37

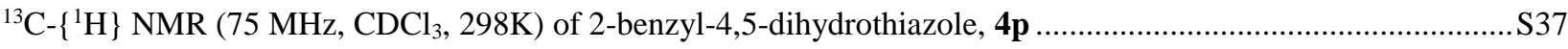

${ }^{1} \mathrm{H}$ NMR (500 MHz, $\mathrm{CDCl}_{3}$, 298K, TMS) of 2-(2-bromobenzyl)-4,5-dihydrothiazole, 4q ................................S38

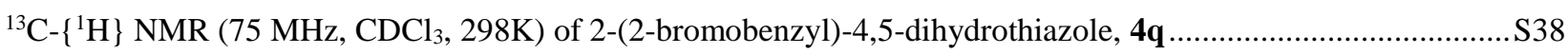

${ }^{1} \mathrm{H}$ NMR (400 MHz, $\mathrm{CDCl}_{3}, 298 \mathrm{~K}$, TMS) of 2-(pyridin-2-yl)-4,5-dihydrothiazole, 4r......................................S39

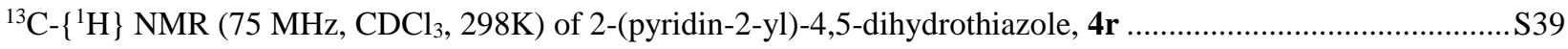

${ }^{1} \mathrm{H}$ NMR (400 MHz, $\mathrm{CDCl}_{3}, 298 \mathrm{~K}$, TMS) of 2-(pyridin-3-yl)-4,5-dihydrothiazole, 4s .......................................S40

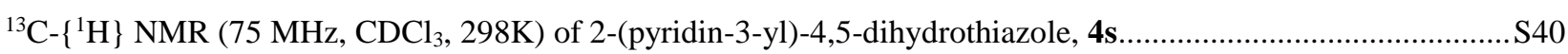

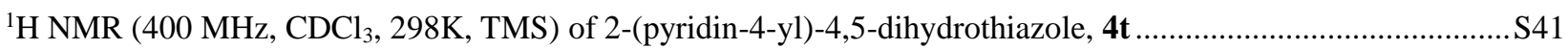

${ }^{13} \mathrm{C}-\left\{{ }^{1} \mathrm{H}\right\}$ NMR $\left(75 \mathrm{MHz}, \mathrm{CDCl}_{3}, 298 \mathrm{~K}\right)$ of 2-(pyridin-4-yl)-4,5-dihydrothiazole, 4t.......................................S41 
${ }^{1} \mathrm{H}$ NMR (400 MHz, $\left.\mathrm{CDCl}_{3}, 298 \mathrm{~K}, \mathrm{TMS}\right)$ of 2-(1H-pyrrol-2-yl)-4,5-dihydrothiazole, 4u. S42

${ }^{13} \mathrm{C}-\left\{{ }^{1} \mathrm{H}\right\}$ NMR $\left(75 \mathrm{MHz}, \mathrm{CDCl}_{3}, 298 \mathrm{~K}\right)$ of 2-(1H-pyrrol-2-yl)-4,5-dihydrothiazole, 4u

${ }^{1} \mathrm{H}$ NMR (400 MHz, $\mathrm{CDCl}_{3}, 298 \mathrm{~K}, \mathrm{TMS}$ ) of 2-(2-furanyl)-4,5-dihydrothiazole, $4 \mathbf{v}$ S43

${ }^{13} \mathrm{C}-\left\{{ }^{1} \mathrm{H}\right\}$ NMR $\left(75 \mathrm{MHz}, \mathrm{CDCl}_{3}, 298 \mathrm{~K}\right)$ of 2-(2-furanyl)-4,5-dihydrothiazole, $4 \mathbf{v}$. S43

${ }^{1} \mathrm{H}$ NMR (400 MHz, $\mathrm{CDCl}_{3}, 298 \mathrm{~K}$, TMS) of 2-(pent-4-yn-1-yl)-4,5-dihydrothiazole, $\mathbf{4 w}$ S44

${ }^{13} \mathrm{C}-\left\{{ }^{1} \mathrm{H}\right\}$ NMR $\left(75 \mathrm{MHz}, \mathrm{CDCl}_{3}, 298 \mathrm{~K}\right)$ of 2-(pent-4-yn-1-yl)-4,5-dihydrothiazole, $\mathbf{4 w}$. S44

${ }^{1} \mathrm{H}$ NMR (400 MHz, $\mathrm{CDCl}_{3}, 298 \mathrm{~K}$, TMS) of 2-pentyl-4,5-dihydrothiazole, 4x S45

${ }^{13} \mathrm{C}$ - $\left\{{ }^{1} \mathrm{H}\right\} \mathrm{NMR}\left(75 \mathrm{MHz}, \mathrm{CDCl}_{3}, 298 \mathrm{~K}\right)$ of 2-pentyl-4,5-dihydrothiazole, $4 \mathbf{x}$ .S45

${ }^{1} \mathrm{H}$ NMR (400 MHz, $\mathrm{CDCl}_{3}, 298 \mathrm{~K}$, TMS) of 2-cyclohexyl-4,5-dihydrothiazole, 4y S46

${ }^{13} \mathrm{C}-\left\{{ }^{1} \mathrm{H}\right\} \mathrm{NMR}\left(75 \mathrm{MHz}, \mathrm{CDCl}_{3}, 298 \mathrm{~K}\right)$ of 2-cyclohexyl-4,5-dihydrothiazole, $4 \mathbf{y}$ S46 


\section{General considerations}

All reactions were carried out in air. All reagents were purchased and used as received. ${ }^{1} \mathrm{H},{ }^{13} \mathrm{C}-\left\{{ }^{1} \mathrm{H}\right\}$ and ${ }^{19} \mathrm{~F}-\left\{{ }^{1} \mathrm{H}\right\}$ Nuclear Magnetic Resonance (NMR) spectra were recorded at 298K on a Brucker Avance 400 Ultrashield or on a Brucker Avance 500 Ultrashield spectrometer using the residual solvent peak for ${ }^{13} \mathrm{C}-\left\{{ }^{1} \mathrm{H}\right\} \mathrm{NMR}\left(\mathrm{CDCl}_{3}: \delta_{\mathrm{C}}=77.16 \mathrm{ppm}\right)$ and TMS as reference for ${ }^{1} \mathrm{H}$ NMR. Gas chromatography (GC) analyses were performed on an Agilent 7890A apparatus equipped with a flame ionization detector and a (5\%-phenyl)-methylpolysiloxane column (30 m, $320 \mu \mathrm{m}$, film: $0.25 \mu \mathrm{m})$. IR measurements were performed on a ReactIR A15 system (Mettler Toledo Autochem) with a DiComp AgX FiberConduit probe $(9.5 \mathrm{~mm})$. Mass spectroscopy was performed by the EPSRC National Mass Spectrometry Service Centre at Swansea University, UK.

\section{Optimisation of reaction conditions}

Table S1. Optimisation of reaction conditions

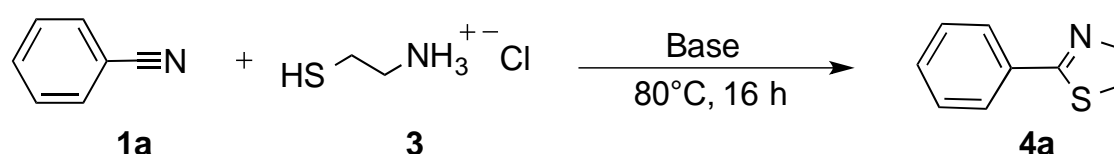

1a

$4 a$

\begin{tabular}{ccccc} 
Entry & $\begin{array}{c}\mathbf{3} \\
(\mathrm{mmol})\end{array}$ & $\begin{array}{c}\text { Base } \\
(\text { Loading })\end{array}$ & $\begin{array}{c}\text { Time } \\
(\mathrm{h})\end{array}$ & $\begin{array}{c}\text { Conversion } \\
(\%)\end{array}$ \\
\hline 1 & 1 & $\mathrm{NaOH}(30 \mathrm{~mol} \%)$ & 16 & $>99$ \\
2 & 1 & $\mathrm{NaOH}(20 \mathrm{~mol} \%)$ & 16 & $>99$ \\
3 & 1 & $\mathrm{NaOH}(10 \mathrm{~mol} \%)$ & 16 & $>99$ \\
4 & 1 & $\mathrm{CsOH}(10 \mathrm{~mol} \%)$ & 16 & $>99$ \\
5 & 1 & $\mathrm{KOH}(10 \mathrm{~mol} \%)$ & 16 & $>99$ \\
6 & 1 & $\mathrm{NaOH}(5 \mathrm{~mol} \%)$ & 16 & $>91$ \\
7 & 0.75 & $\mathrm{NaOH}(20 \mathrm{~mol} \%)$ & 16 & $>99(\mathbf{9 8})^{c}$ \\
8 & 0.75 & $\mathrm{NaOH}(20 \mathrm{~mol} \%)$ & $\mathbf{2}$ & 94 \\
9 & 0.75 & $\mathrm{NaOH}(20 \mathrm{~mol} \%)$ & 1 &
\end{tabular}

${ }^{a}$ Reaction conditions: $1(0.5 \mathrm{mmol}), \mathbf{3}(0.75-1 \mathrm{mmol})$, base-catalyst, $80^{\circ} \mathrm{C}, 1-16 \mathrm{~h} .{ }^{b}$ Conversion determined by GC, based on benzonitrile, average of 2 reactions. ${ }^{c}$ Isolated yield. 
Table S2. Comparison of sources of $\mathrm{NaOH}^{a}$

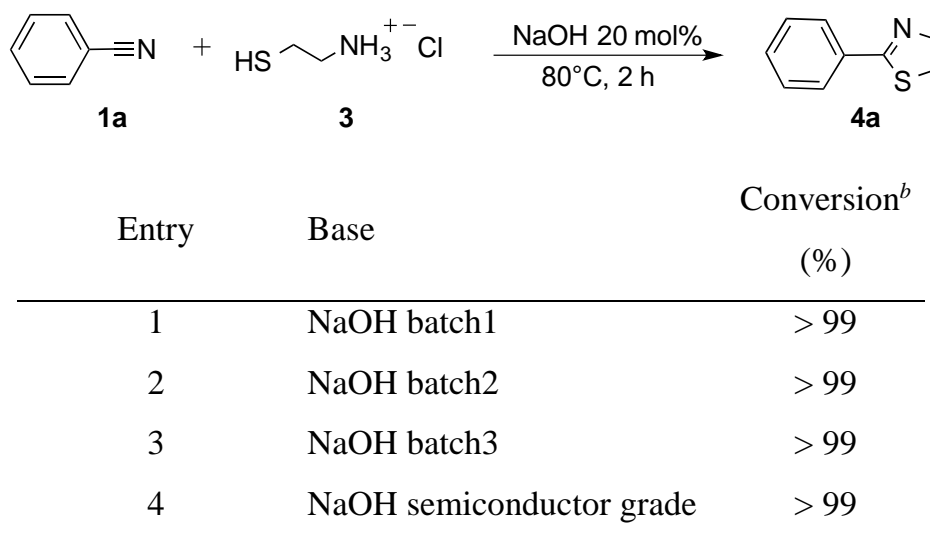

${ }^{a}$ Reaction conditions: 1 (0.5 mmol), $3(0.75 \mathrm{mmol}), \mathrm{NaOH}(20 \mathrm{~mol} \%), 80^{\circ} \mathrm{C}, 2 \mathrm{~h}$.

${ }^{b}$ Conversion determined by GC, based on benzonitrile, average of 2 reactions. 


\section{In Situ FTIR Spectroscopy}

Figure S1 IR spectra of benzonitrile, 1a

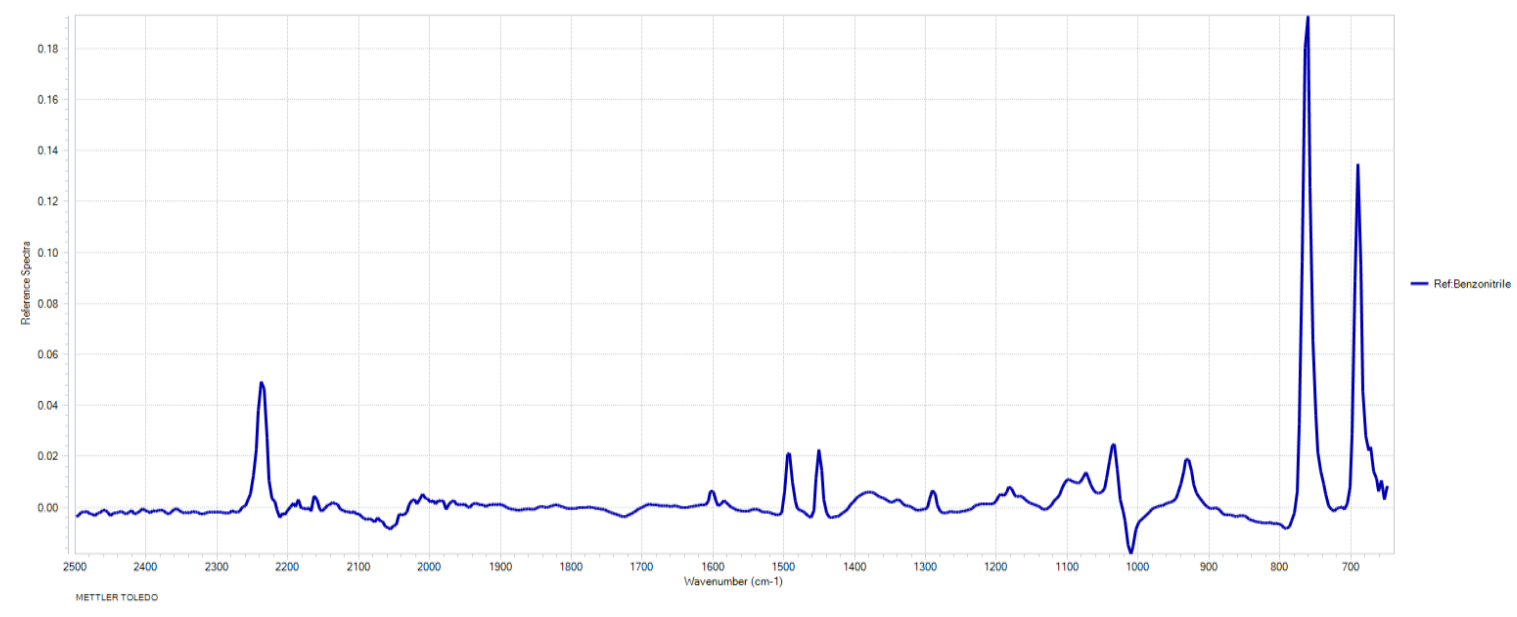

Figure S2 IR spectra of 2-Phenyl-4,5-dihydrothiazole, 4a

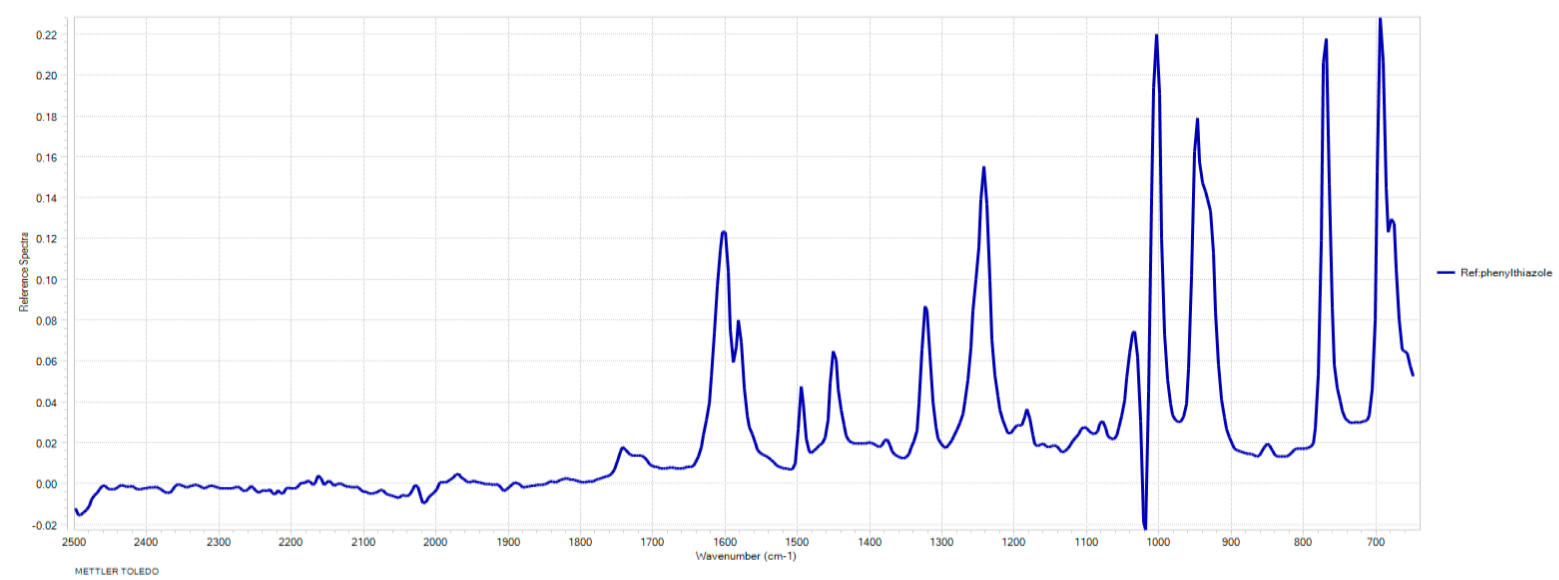


Reaction between benzonitrile and cysteamine hydrochloride without base

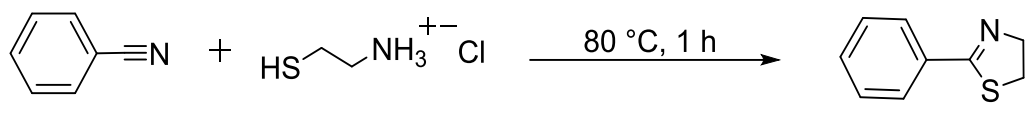

1a

3

$4 a$

PROCEDURE: In air, a $5 \mathrm{~mL}$ round bottom flask was charged with benzonitrile $(19.4 \mathrm{mmol}, 2.0 \mathrm{~g})$ and cysteamine hydrochloride $(29.1 \mathrm{mmol}, 3.3 \mathrm{~g})$. The mixture was heated at $80^{\circ} \mathrm{C}$ until cysteamine hydrochloride was completely melted. The probe was submerged in the reaction mixture and the IR measurements were started. The reaction was stirred (350 rpm) at $80^{\circ} \mathrm{C}$ for $1 \mathrm{~h}$ under solvent-free conditions.

Figure S3 3D plot of in situ IR measurements of the reaction between $1 \mathbf{a}$ and $\mathbf{3}$ without base.

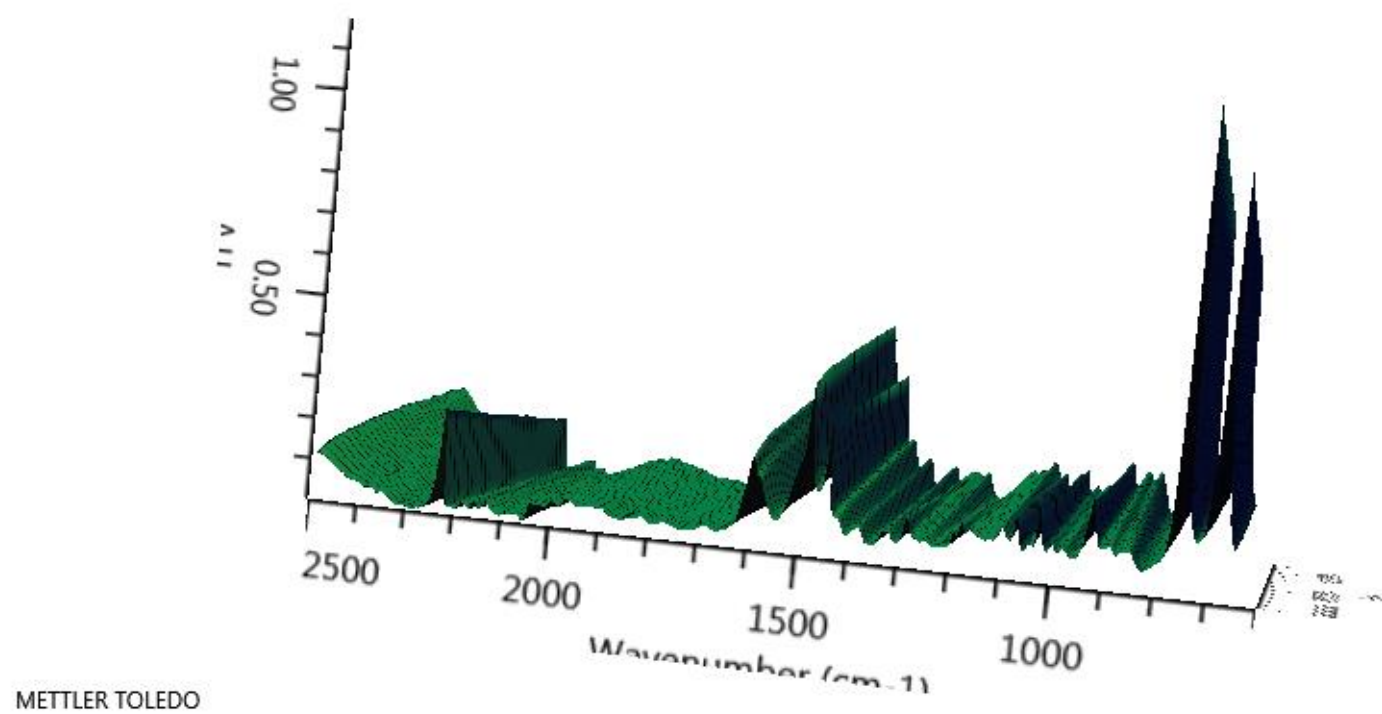


Figure S4 IR plot of the reaction between 1a and 3 without base.

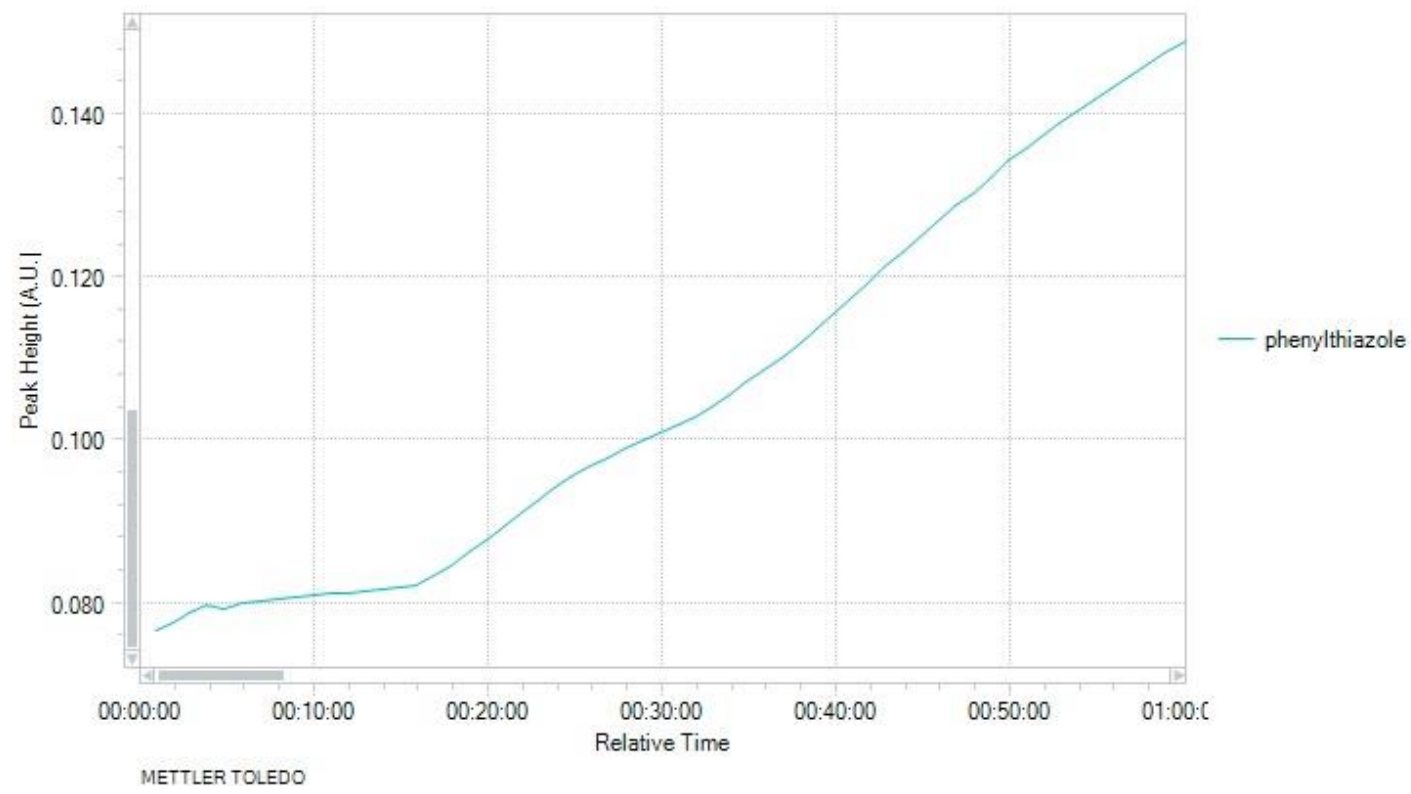




\section{Reaction between benzonitrile and cysteamine hydrochloride in the presence of $\mathrm{NaOH}$}

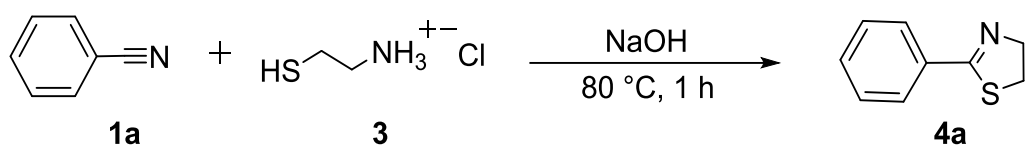

PROCEDURE: In air, a $5 \mathrm{~mL}$ round bottom flask was charged with benzonitrile $(19.4 \mathrm{mmol}, 2.0 \mathrm{~g})$ and cysteamine hydrochloride $(29.1 \mathrm{mmol}, 3.3 \mathrm{~g})$. The mixture was heated at $80{ }^{\circ} \mathrm{C}$ until cysteamine hydrochloride was completely melted. The probe was submerged inside of the reaction mixture and the IR measurements were started. After 5 minutes $20 \mathrm{~mol} \%$ of $\mathrm{NaOH}(2 \mathrm{mmol}, 80 \mathrm{mg})$ were added. The reaction was stirred $(350 \mathrm{rpm})$ at $80{ }^{\circ} \mathrm{C}$ for $1 \mathrm{~h}$ under solvent-free conditions.

Figure S5 3D plot of in situ IR measurements of the reaction between 1a and $\mathbf{3}$ in the presence of $\mathrm{NaOH}$.

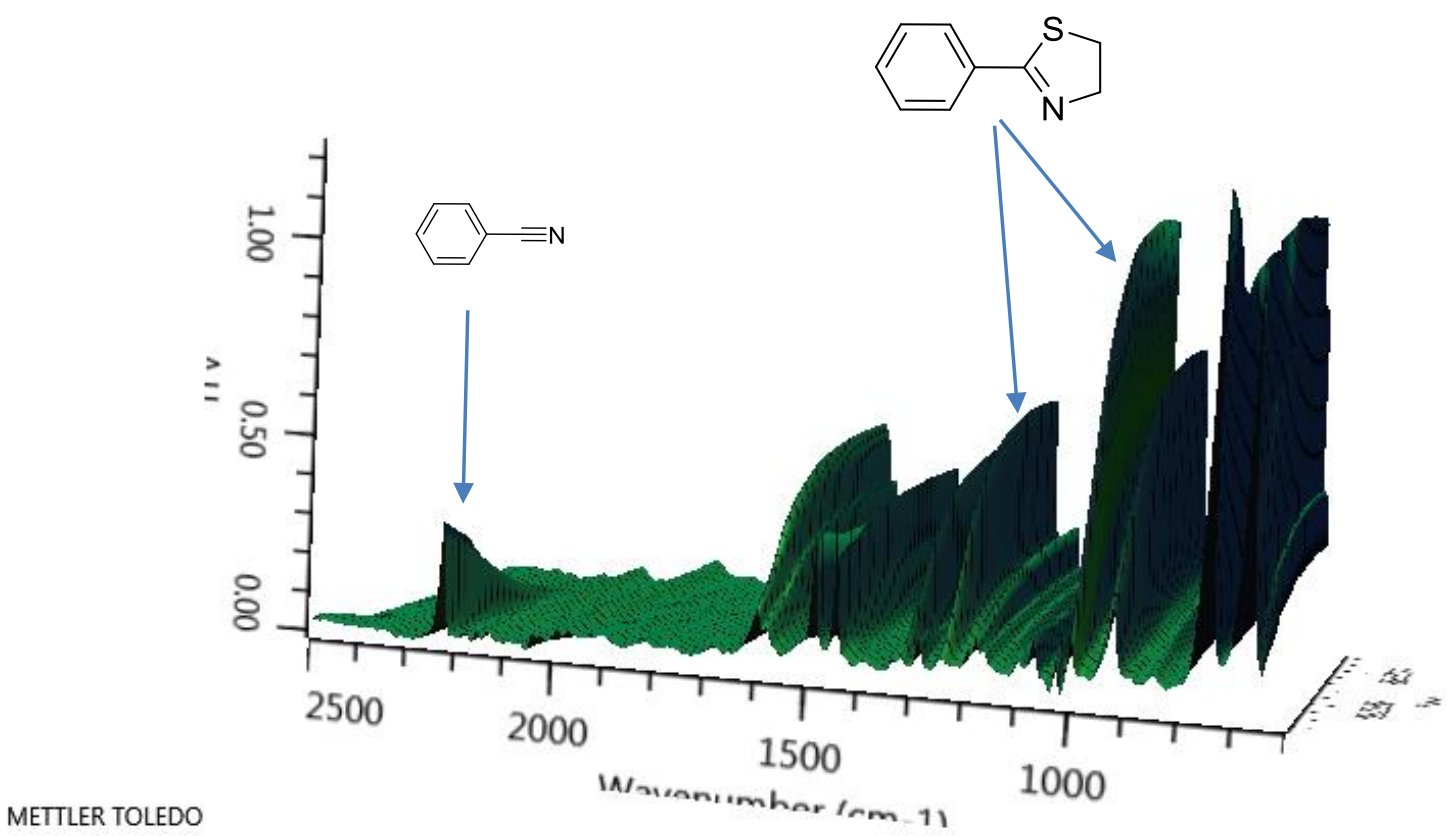


Figure S6 IR plot of the reaction between $\mathbf{1 a}$ and $\mathbf{3}$ in the presence of $\mathrm{NaOH}$.

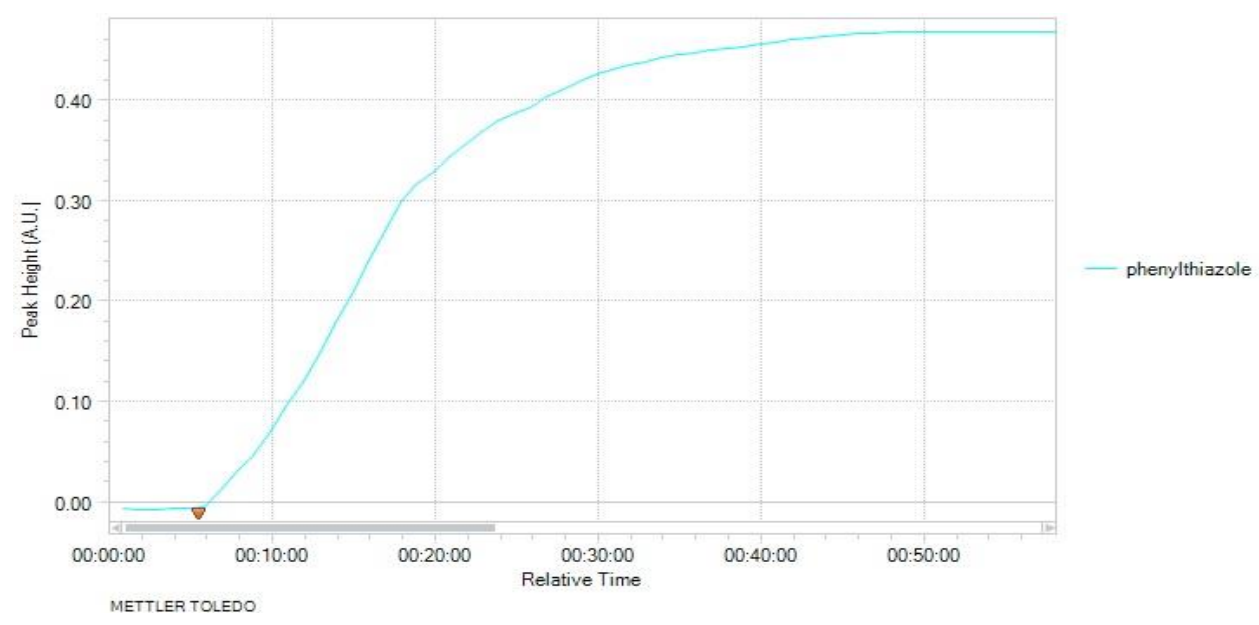

\section{$4 \quad$ Synthesis and characterisation of thiazolines}

In air, a vial was charged with the nitrile $(1 \mathrm{mmol})$, cysteamine hydrochloride $(1.5 \mathrm{mmol})$ and $\mathrm{NaOH}(0.2 \mathrm{mmol})$. The reaction was stirred at $80{ }^{\circ} \mathrm{C}$ for the appropriate time under solvent-free conditions. The crude product was dissolved in ethyl acetate $(2 \mathrm{~mL})$ and water $(10 \mathrm{~mL})$ was added. The aqueous layer was then extracted with ethyl acetate $(3 \mathrm{x} 10 \mathrm{~mL})$. The combined organic layers were dried over $\mathrm{MgSO}_{4}$, filtered and dried under vacuum to give the desired compound. The conversion was determined by GC analysis or by ${ }^{1} \mathrm{H}$ NMR.

2-Phenyl-4,5-dihydrothiazole, $\mathbf{4} \mathbf{a}^{[1]}$<smiles>c1ccc(C2=NCCS2)cc1</smiles>

The general procedure yielded to the title compound as a yellow oil (153 $\mathrm{mg}, 98 \%)$.

${ }^{1}$ H NMR (500 MHz, CDCl3, 298K, TMS): $\delta(\mathrm{ppm})=7.85-7.82\left(\mathrm{~m}, 2 \mathrm{H}, \mathrm{C}_{\mathrm{Ar}} H\right), 7.47-7.38\left(\mathrm{~m}, 3 \mathrm{H}, \mathrm{C}_{\mathrm{Ar}} H\right) 4.45\left(\mathrm{t},{ }^{3} \mathrm{~J}_{\mathrm{HH}}=\right.$ $\left.8.4 \mathrm{~Hz}, 2 \mathrm{H}, \mathrm{CH}_{2}\right), 3.40\left(\mathrm{t},{ }^{3} \mathrm{~J}_{\mathrm{HH}}=8.4 \mathrm{~Hz}, 2 \mathrm{H}, \mathrm{CH}_{2}\right)$.

${ }^{13} \mathrm{C}-\left\{{ }^{1} \mathrm{H}\right\}$ NMR (125.7 MHz, $\left.\mathbf{C D C l}_{3}, \mathbf{2 9 8 K}\right): \delta(\mathrm{ppm})=168.6\left(\mathrm{~s}, \mathrm{C}^{\mathrm{IV}}\right), 133.5\left(\mathrm{~s}, \mathrm{C}^{\mathrm{IV}}\right), 131.3\left(\mathrm{~s}, C_{\mathrm{Ar}} \mathrm{H}\right), 128.7\left(\mathrm{~s}, C_{\mathrm{Ar}} \mathrm{H}\right)$, $128.6\left(\mathrm{~s}, \mathrm{C}_{\mathrm{Ar}} \mathrm{H}\right), 65.5\left(\mathrm{~s}, \mathrm{CH}_{2}\right), 33.9\left(\mathrm{~s}, \mathrm{CH}_{2}\right)$.

2-(4-Fluorophenyl)-4,5-dihydrothiazole, $\mathbf{4 b}^{[2]}$<smiles>Fc1ccc(C2=NCCS2)cc1</smiles>

The general procedure yielded to the title compound as a colourless oil (168 mg, 93\%).

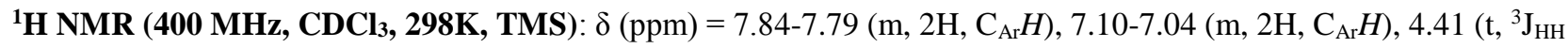
$\left.=8.3 \mathrm{~Hz}, 2 \mathrm{H}, \mathrm{CH}_{2}\right), 3.38\left(\mathrm{t},{ }^{3} \mathrm{~J}_{\mathrm{HH}}=8.3 \mathrm{~Hz}, 2 \mathrm{H}, \mathrm{CH}_{2}\right)$.

${ }^{13} \mathrm{C}-\left\{{ }^{1} \mathbf{H}\right\}$ NMR (75 MHz, CDCl $\left.3,298 K\right): \delta(p p m)=167.1\left(\mathrm{~s}, \mathrm{C}^{\mathrm{IV}}\right), 164.5\left(\mathrm{~d},{ }^{1} \mathrm{~J}_{\mathrm{CF}}=251.5 \mathrm{~Hz}, \mathrm{C}^{\mathrm{IV}}\right), 130.5\left(\mathrm{~d},{ }^{4} \mathrm{~J}_{\mathrm{CF}}=8.7\right.$ $\left.\mathrm{Hz}, C_{\mathrm{Ar}} \mathrm{H}\right), 129.7\left(\mathrm{~d},{ }^{5} \mathrm{~J}_{\mathrm{CF}}=3.6 \mathrm{~Hz}, \mathrm{C}^{\mathrm{IV}}\right), 115.6\left(\mathrm{~d},{ }^{3} \mathrm{~J}_{\mathrm{CF}}=22.2 \mathrm{~Hz}, C_{\mathrm{Ar}} \mathrm{H}\right), 65.3\left(\mathrm{~s}, C \mathrm{H}_{2}\right), 34.0\left(\mathrm{~s}, C \mathrm{H}_{2}\right)$. 
${ }^{19} \mathbf{F}-\left\{{ }^{1} \mathbf{H}\right\}$ NMR (376 MHz, 298K): $\delta(\mathrm{ppm})=-108.73(\mathrm{~s}, \mathrm{CF})$

2-(4-Chlorophenyl)-4,5-dihydrothiazole, $\mathbf{4} \mathbf{c}^{[1]}$<smiles>Clc1ccc(C2=NCCS2)cc1</smiles>

The general procedure yielded to the title compound as a colourless solid (179 mg, 91\%).

${ }^{1} \mathrm{H}$ NMR (400 MHz, $\mathbf{C D C l}_{3}$, 298K, TMS): $\delta(\mathrm{ppm})=7.78-7.75\left(\mathrm{~m}, 2 \mathrm{H}, \mathrm{C}_{\mathrm{Ar}} H\right), 7.39-7.37$ (m, 2H, $\left.\mathrm{C}_{\mathrm{Ar}} H\right), 4.47-4.43$ (m, $\left.2 \mathrm{H}, \mathrm{CH}_{2}\right), 3.45-3.40\left(\mathrm{~m}, 2 \mathrm{H}, \mathrm{CH}_{2}\right)$.

${ }^{13} \mathrm{C}-\left\{{ }^{1} \mathrm{H}\right\}$ NMR (75 MHz, $\left.\mathbf{C D C l}_{3}, \mathbf{2 9 8 K}\right): \delta(\mathrm{ppm})=167.6\left(\mathrm{~s}, \mathrm{C}^{\mathrm{IV}}\right), 137.5\left(\mathrm{~s}, \mathrm{C}^{\mathrm{IV}}\right), 132.0\left(\mathrm{~s}, \mathrm{C}^{\mathrm{IV}}\right), 129.9\left(\mathrm{~s}, C_{\mathrm{Ar}} \mathrm{H}\right), 129.0$ (s, $\left.\mathrm{C}_{\mathrm{Ar}} \mathrm{H}\right), 65.6$ (s, $\left.\mathrm{CH}_{2}\right), 34.2\left(\mathrm{~s}, \mathrm{CH}_{2}\right)$.

Melting point: $53^{\circ} \mathrm{C}$

2-(4-Bromophenyl)-4,5-dihydrothiazole, $\mathbf{4 d}^{[3]}$<smiles>Brc1ccc(C2=NCCS2)cc1</smiles>

The general procedure yielded to the title compound as a colourless solid (228 $\mathrm{mg}, 94 \%)$.

${ }^{1} \mathrm{H}$ NMR (400 MHz, $\left.\mathbf{C D C l}_{3}, \mathbf{2 9 8 K}, \mathbf{T M S}\right): \delta(\mathrm{ppm})=7.71-7.69\left(\mathrm{~m}, 2 \mathrm{H}, \mathrm{C}_{\mathrm{Ar}} H\right), 7.56-7.53\left(\mathrm{~m}, 2 \mathrm{H}, \mathrm{C}_{\mathrm{Ar}} H\right), 4.44\left(\mathrm{t},{ }^{3} \mathrm{~J}_{\mathrm{HH}}\right.$ $\left.=8.4 \mathrm{~Hz}, 2 \mathrm{H}, \mathrm{CH}_{2}\right), 3.45-3.41\left(\mathrm{~m}, 2 \mathrm{H}, \mathrm{CH}_{2}\right)$.

${ }^{13} \mathrm{C}-\left\{{ }^{1} \mathrm{H}\right\}$ NMR (75 MHz, $\left.\mathbf{C D C l}_{3}, \mathbf{2 9 8 K}\right): \delta(\mathrm{ppm})=167.7\left(\mathrm{~s}, \mathrm{C}^{\mathrm{IV}}\right), 132.5\left(\mathrm{~s}, \mathrm{C}^{\mathrm{IV}}\right), 132.0\left(\mathrm{~s}, C_{\mathrm{Ar}} \mathrm{H}\right), 130.1\left(\mathrm{~s}, C_{\mathrm{Ar}} \mathrm{H}\right)$, $126.0\left(\mathrm{~s}, \mathrm{C}^{\mathrm{IV}}\right), 65.6\left(\mathrm{~s}, \mathrm{CH}_{2}\right), 34.3\left(\mathrm{~s}, \mathrm{CH}_{2}\right)$.

Melting point: $91^{\circ} \mathrm{C}$

2-p-Tolyl-4,5-dihydrothiazole, $4 \mathbf{e}^{[1]}$<smiles>Cc1ccc(C2=NCCS2)cc1</smiles>

The general procedure yielded to the title compound as a colourless solid (158 mg, 89\%).

${ }^{1} \mathrm{H}$ NMR (400 MHz, $\left.\mathbf{C D C l}_{3}, \mathbf{2 9 8 K}, \mathbf{T M S}\right): \delta(\mathrm{ppm})=7.74-7.71\left(\mathrm{~m}, 2 \mathrm{H}, \mathrm{C}_{\mathrm{Ar}} H\right), 7.21-7.19\left(\mathrm{~m}, 2 \mathrm{H}, \mathrm{C}_{\mathrm{Ar}} H\right), 4.43\left(\mathrm{t},{ }^{3} \mathrm{~J}_{\mathrm{HH}}\right.$ $\left.=8.3 \mathrm{~Hz}, 2 \mathrm{H}, \mathrm{CH}_{2}\right), 3.38\left(\mathrm{t},{ }^{3} \mathrm{~J}_{\mathrm{HH}}=8.3 \mathrm{~Hz}, 2 \mathrm{H}, \mathrm{CH}_{2}\right), 2.37\left(\mathrm{~s}, 3 \mathrm{H}, \mathrm{CH}_{3}\right)$.

${ }^{13} \mathrm{C}-\left\{{ }^{1} \mathrm{H}\right\}$ NMR (75 MHz, $\left.\mathbf{C D C l}_{3}, \mathbf{2 9 8 K}\right): \delta(\mathrm{ppm})=168.6\left(\mathrm{~s}, \mathrm{C}^{\mathrm{IV}}\right), 141.7\left(\mathrm{~s}, \mathrm{C}^{\mathrm{IV}}\right), 130.9\left(\mathrm{~s}, \mathrm{C}^{\mathrm{IV}}\right), 129.4\left(\mathrm{~s}, C_{\mathrm{Ar}} \mathrm{H}\right), 128.6$ (s, $\left.C_{\mathrm{Ar}} \mathrm{H}\right), 65.4$ (s, $\left.\mathrm{CH}_{2}\right), 33.9\left(\mathrm{~s}, \mathrm{CH}_{2}\right), 21.7$ (s, $\left.\mathrm{CH}_{3}\right)$.

Melting point: $42^{\circ} \mathrm{C}$ 
2-(4-Methoxyphenyl)-4,5-dihydrothiazole, $\mathbf{4 f}^{[3]}$<smiles>COc1ccc(C2=NCCS2)cc1</smiles>

The general procedure yielded to the title compound as a yellow solid (188 mg, 97\%).

${ }^{1} \mathrm{H}$ NMR (500 MHz, $\left.\mathbf{C D C l}_{3}, \mathbf{2 9 8 K}, \mathbf{T M S}\right): \delta(\mathrm{ppm})=7.79-7.76\left(\mathrm{~m}, 2 \mathrm{H}, \mathrm{C}_{\mathrm{Ar}} H\right), 6.90-6.88\left(\mathrm{~m}, 2 \mathrm{H}, \mathrm{C}_{\mathrm{Ar}} H\right), 4.39\left(\mathrm{t},{ }^{3} \mathrm{~J}_{\mathrm{HH}}\right.$ $\left.=8.2 \mathrm{~Hz}, 2 \mathrm{H}, \mathrm{CH}_{2}\right), 3.80\left(\mathrm{~s}, 3 \mathrm{H}, \mathrm{OCH}_{3}\right), 3.35\left(\mathrm{t},{ }^{3} \mathrm{~J}_{\mathrm{HH}}=8.2 \mathrm{~Hz}, 2 \mathrm{H}, \mathrm{CH}_{2}\right)$.

${ }^{13} \mathrm{C}-\left\{{ }^{1} \mathrm{H}\right\}$ NMR $\left(125.7 \mathrm{MHz}, \mathbf{C D C l}_{3}, \mathbf{2 9 8 K}\right): \delta(\mathrm{ppm})=167.7\left(\mathrm{~s}, \mathrm{C}^{\mathrm{IV}}\right), 162.0\left(\mathrm{~s}, \mathrm{C}^{\mathrm{IV}}\right), 130.1\left(\mathrm{~s}, C_{\mathrm{Ar}} \mathrm{H}\right), 126.2\left(\mathrm{~s}, \mathrm{C}^{\mathrm{IV}}\right)$, $113.9\left(\mathrm{~s}, C_{\mathrm{Ar}} \mathrm{H}\right), 65.2\left(\mathrm{~s}, \mathrm{CH}_{2}\right), 55.5\left(\mathrm{~s}, \mathrm{OCH}_{3}\right), 33.8\left(\mathrm{~s}, \mathrm{CH}_{2}\right)$.

Melting point: $53{ }^{\circ} \mathrm{C}$

4-(4,5-Dihydrothiazol-2-yl)aniline, $\mathbf{4 g}^{[4]}$<smiles>Nc1ccc(C2=NCCS2)cc1</smiles>

The general procedure yielded to the title compound as a pale yellow oil (166 mg, 93\%).

${ }^{1} \mathrm{H}$ NMR (400 MHz, $\mathbf{C D C l}_{3}$, 298K, TMS): $\delta(\mathrm{ppm})=7.65-7.63\left(\mathrm{~m}, 2 \mathrm{H}, \mathrm{C}_{\mathrm{Ar}} H\right), 6.64-6.61$ (m, 2H, $\left.\mathrm{C}_{\mathrm{Ar}} H\right), 4.40-4.36(\mathrm{~m}$, $\left.2 \mathrm{H}, \mathrm{CH}_{2}\right), 3.98\left(\mathrm{~s}, 2 \mathrm{H}, \mathrm{NH}_{2}\right), 3.36-3.32\left(\mathrm{~m}, 2 \mathrm{H}, \mathrm{CH}_{2}\right)$.

${ }^{13} \mathrm{C}-\left\{{ }^{1} \mathrm{H}\right\}$ NMR (75 MHz, CDCl $\left.3,298 K\right): \delta(\mathrm{ppm})=168.2\left(\mathrm{~s}, \mathrm{C}^{\mathrm{IV}}\right), 149.6\left(\mathrm{~s}, \mathrm{C}^{\mathrm{IV}}\right), 130.3\left(\mathrm{~s}, C_{\mathrm{Ar}} \mathrm{H}\right), 123.7\left(\mathrm{~s}, \mathrm{C}^{\mathrm{IV}}\right), 114.5$ (s, $\left.\mathrm{C}_{\mathrm{Ar}} \mathrm{H}\right), 65.1$ (s, $\left.\mathrm{CH}_{2}\right), 33.7$ (s, $\left.\mathrm{CH}_{2}\right)$.

2-(3-Bromophenyl)-4,5-dihydrothiazole, $\mathbf{4 h} \mathbf{h}^{[5]}$<smiles>Brc1cccc(C2=NCCS2)c1</smiles>

The general procedure yielded to the title compound as a pale brown solid (226 mg, 94\%).

${ }^{1} \mathbf{H}$ NMR (400 MHz, CDCl 3 , 298K, TMS): $\delta(\mathrm{ppm})=8.00\left(\mathrm{~d},{ }^{5} \mathrm{~J}_{\mathrm{HH}}=1.9 \mathrm{~Hz}, 1 \mathrm{H}, \mathrm{C}_{\mathrm{Ar}} H\right), 7.72\left(\mathrm{dt},{ }^{3} \mathrm{~J}_{\mathrm{HH}}=7.8 \mathrm{~Hz},{ }^{5} \mathrm{~J}_{\mathrm{HH}}=\right.$ $\left.1.4 \mathrm{~Hz}, 1 \mathrm{H}, \mathrm{C}_{\mathrm{Ar}} H\right), 7.57\left(\mathrm{dq},{ }^{3} \mathrm{~J}_{\mathrm{HH}}=7.8 \mathrm{~Hz},{ }^{5} \mathrm{~J}_{\mathrm{HH}}=1.2 \mathrm{~Hz}, 1 \mathrm{H}, \mathrm{C}_{\mathrm{Ar}} H\right), 7.26\left(\mathrm{t},{ }^{3} \mathrm{~J}_{\mathrm{HH}}=7.8 \mathrm{~Hz}, 1 \mathrm{H}, \mathrm{C}_{\mathrm{Ar}} H\right), 4.44\left(\mathrm{t},{ }^{3} \mathrm{~J}_{\mathrm{HH}}=\right.$ $\left.8.4 \mathrm{~Hz}, 2 \mathrm{H}, \mathrm{CH}_{2}\right), 3.42\left(\mathrm{t},{ }^{3} \mathrm{~J}_{\mathrm{HH}}=8.4 \mathrm{~Hz}, 2 \mathrm{H}, \mathrm{CH}_{2}\right)$.

${ }^{13} \mathrm{C}-\left\{{ }^{1} \mathbf{H}\right\}$ NMR (75 MHz, $\left.\mathbf{C D C l}_{3}, \mathbf{2 9 8 K}\right): \delta(\mathrm{ppm})=167.2\left(\mathrm{~s}, \mathrm{C}^{\mathrm{IV}}\right), 135.4\left(\mathrm{~s}, \mathrm{C}^{\mathrm{IV}}\right), 134.2\left(\mathrm{~s}, C_{\mathrm{Ar}} \mathrm{H}\right), 131.4\left(\mathrm{~s}, C_{\mathrm{Ar}} \mathrm{H}\right)$, $130.2\left(\mathrm{~s}, C_{\mathrm{Ar}} \mathrm{H}\right), 127.3\left(\mathrm{~s}, C_{\mathrm{Ar}} \mathrm{H}\right), 122.8\left(\mathrm{~s}, \mathrm{C}^{\mathrm{IV}}\right), 65.5\left(\mathrm{~s}, \mathrm{CH}_{2}\right), 34.2\left(\mathrm{~s}, \mathrm{CH}_{2}\right)$.

Melting point: $45^{\circ} \mathrm{C}$ 
2-m-Tolyl-4,5-dihydrothiazole, $\mathbf{4 i}^{[6]}$<smiles>Cc1cccc(C2=NCCS2)c1</smiles>

The general procedure yielded to the title compound as a colourless oil (150 mg, 85\%).

${ }^{1} \mathrm{H}$ NMR (500 MHz, $\mathbf{C D C l}_{3}$, 298K, TMS): $\delta(\mathrm{ppm})=7.67\left(\mathrm{~s}, 1 \mathrm{H}, \mathrm{C}_{\mathrm{Ar}} H\right), 7.62\left(\mathrm{~d},{ }^{3} \mathrm{~J}_{\mathrm{HH}}=7.3 \mathrm{~Hz}, 1 \mathrm{H}, \mathrm{C}_{\mathrm{Ar}} H\right), 7.30-7.24$ $\left(\mathrm{m}, 2 \mathrm{H}, \mathrm{C}_{\mathrm{Ar}} \mathrm{H}\right), 4.43\left(\mathrm{t},{ }^{3} \mathrm{~J}_{\mathrm{HH}}=8.4 \mathrm{~Hz}, 2 \mathrm{H}, \mathrm{CH}_{2}\right), 3.38\left(\mathrm{t},{ }^{3} \mathrm{~J}_{\mathrm{HH}}=8.4 \mathrm{~Hz}, 2 \mathrm{H}, \mathrm{CH}_{2}\right), 2.38\left(\mathrm{~s}, 3 \mathrm{H}, \mathrm{CH}_{3}\right)$.

${ }^{13} \mathrm{C}-\left\{{ }^{1} \mathrm{H}\right\}$ NMR $\left(125.7 \mathrm{MHz}, \mathbf{C D C l}_{3}, \mathbf{2 9 8 K}\right): \delta(\mathrm{ppm})=168.8\left(\mathrm{~s}, \mathrm{C}^{\mathrm{IV}}\right), 138.5\left(\mathrm{~s}, \mathrm{C}^{\mathrm{IV}}\right), 133.4\left(\mathrm{~s}, \mathrm{C}^{\mathrm{IV}}\right), 132.1\left(\mathrm{~s}, C_{\mathrm{Ar}} \mathrm{H}\right)$, $129.0\left(\mathrm{~s}, C_{\mathrm{Ar}} \mathrm{H}\right), 128.6\left(\mathrm{~s}, C_{\mathrm{Ar}} \mathrm{H}\right), 125.9\left(\mathrm{~s}, C_{\mathrm{Ar}} \mathrm{H}\right), 65.4\left(\mathrm{~s}, \mathrm{CH}_{2}\right), 33.8\left(\mathrm{~s}, \mathrm{CH}_{2}\right), 21.5\left(\mathrm{~s}, \mathrm{CH}_{3}\right)$.

3-(4,5-Dihydrothiazol-2-yl)aniline, $\mathbf{4 j}^{[1]}$<smiles>Nc1cccc(C2=NCCS2)c1</smiles>

The general procedure yielded to the title compound as a brown solid (176 mg, 99\%).

${ }^{1} \mathrm{H}$ NMR (400 MHz, $\left.\mathbf{C D C l}_{3}, \mathbf{2 9 8 K}, \mathbf{T M S}\right): \delta(\mathrm{ppm})=7.20-7.13\left(\mathrm{~m}, 3 \mathrm{H}, \mathrm{C}_{\mathrm{Ar}} H\right), 6.74-6.71\left(\mathrm{~m}, 1 \mathrm{H}, \mathrm{C}_{\mathrm{Ar}} H\right), 4.40\left(\mathrm{t},{ }^{3} \mathrm{~J}_{\mathrm{HH}}\right.$ $\left.=8.4 \mathrm{~Hz}, 2 \mathrm{H}, \mathrm{CH}_{2}\right), 3.79\left(\mathrm{~s}, 2 \mathrm{H}, \mathrm{NH}_{2}\right), 3.35\left(\mathrm{t},{ }^{3} \mathrm{~J}_{\mathrm{HH}}=8.4 \mathrm{~Hz}, 2 \mathrm{H}, \mathrm{CH}_{2}\right)$.

${ }^{13} \mathrm{C}-\left\{{ }^{1} \mathrm{H}\right\}$ NMR $\left(\mathbf{7 5} \mathrm{MHz}, \mathbf{C D C l}_{3}, \mathbf{2 9 8 K}\right): \delta(\mathrm{ppm})=168.8\left(\mathrm{~s}, \mathrm{C}^{\mathrm{IV}}\right), 146.8\left(\mathrm{~s}, \mathrm{C}^{\mathrm{IV}}\right), 134.3\left(\mathrm{~s}, \mathrm{C}^{\mathrm{IV}}\right), 129.5\left(\mathrm{~s}, C_{\mathrm{Ar}} \mathrm{H}\right), 118.9$ $\left(\mathrm{s}, C_{\mathrm{Ar}} \mathrm{H}\right), 117.9\left(\mathrm{~s}, C_{\mathrm{Ar}} \mathrm{H}\right), 114.4\left(\mathrm{~s}, C_{\mathrm{Ar}} \mathrm{H}\right), 65.2\left(\mathrm{~s}, \mathrm{CH}_{2}\right), 33.7\left(\mathrm{~s}, C_{2}\right)$.

Melting point: $66^{\circ} \mathrm{C}$

2-(3,5-Difluorophenyl)-4,5-dihydrothiazole, $4 \mathbf{k}$<smiles>Fc1cc(F)cc(C2=NCCS2)c1</smiles>

The general procedure yielded to the title compound as a colourless solid (194 mg, 98\%).

${ }^{1} \mathbf{H}$ NMR (400 MHz, $\left.\mathbf{C D C l}_{3}, \mathbf{2 9 8 K}, \mathbf{T M S}\right): \delta(\mathrm{ppm})=7.38-7.33\left(\mathrm{~m}, 2 \mathrm{H}, \mathrm{C}_{\mathrm{Ar}} H\right), 6.93-6.88\left(\mathrm{~m}, 1 \mathrm{H}, \mathrm{C}_{\mathrm{Ar}} H\right), 4.46\left(\mathrm{t},{ }^{3} \mathrm{~J}_{\mathrm{HH}}\right.$ $\left.=8.5 \mathrm{~Hz}, 2 \mathrm{H}, \mathrm{CH}_{2}\right), 3.45\left(\mathrm{t},{ }^{3} \mathrm{~J}_{\mathrm{HH}}=8.5 \mathrm{~Hz}, 2 \mathrm{H}, \mathrm{CH}_{2}\right)$.

${ }^{13} \mathbf{C}-\left\{{ }^{1} \mathbf{H}\right\}$ NMR (75 MHz, CDCl $\left.3,298 K\right): \delta(\mathrm{ppm})=166.5\left(\mathrm{~m}, \mathrm{C}^{\mathrm{IV}}\right), 163.0\left(\mathrm{dd},{ }^{1} \mathrm{~J}_{\mathrm{CF}}=249.9 \mathrm{~Hz},{ }^{3} \mathrm{~J}_{\mathrm{CF}}=14.2 \mathrm{~Hz}, \mathrm{C}^{\mathrm{IV}}\right)$, $136.5\left(\mathrm{t},{ }^{3} \mathrm{~J}_{\mathrm{CF}}=10.2 \mathrm{~Hz}, \mathrm{C}^{\mathrm{IV}}\right), 111.6\left(\mathrm{dd},{ }^{2} \mathrm{~J}_{\mathrm{CF}}=19.7 \mathrm{~Hz},{ }^{4} \mathrm{~J}_{\mathrm{CF}}=7.9 \mathrm{~Hz}, C_{\mathrm{Ar}} \mathrm{H}\right), 106.6\left(\mathrm{t},{ }^{2} \mathrm{~J}_{\mathrm{CF}}=25.6 \mathrm{~Hz}, C_{\mathrm{Ar}} \mathrm{H}\right), 65.5(\mathrm{~s}$, $\left.\mathrm{CH}_{2}\right), 34.4$ (s, $\mathrm{CH}_{2}$ ).

${ }^{19} \mathbf{F}-\left\{{ }^{1} \mathbf{H}\right\}$ NMR (376 MHz, 298K): $\delta(\mathrm{ppm})=-109.01(\mathrm{~s}, \mathrm{CF})$.

HRMS calcd. for $\mathbf{C}_{9} \mathbf{H}_{8} \mathbf{F}_{2} \mathbf{N S}(\mathbf{M}+\mathbf{H})^{+} 200.0340$ found 200.0342 .

Melting point: $43^{\circ} \mathrm{C}$ 
2-(2-Chlorophenyl)-4,5-dihydrothiazole, $\mathbf{4 l}^{[1]}$<smiles>Clc1ccccc1C1=NCCS1</smiles>

The general procedure yielded to the title compound as a light yellow oil (180 mg, 91\%).

${ }^{1} \mathbf{H}$ NMR $\left(500 \mathrm{MHz}, \mathbf{C D C l}_{3}, \mathbf{2 9 8 K}\right.$, TMS): $\delta(\mathrm{ppm})=7.60\left(\mathrm{dd},{ }^{3} \mathrm{~J}_{\mathrm{HH}}=7.6 \mathrm{~Hz},{ }^{5} \mathrm{~J}_{\mathrm{HH}}=1.8 \mathrm{~Hz}, 1 \mathrm{H}, \mathrm{C}_{\mathrm{Ar}} H\right), 7.42\left(\mathrm{dd},{ }^{3} \mathrm{~J}_{\mathrm{HH}}\right.$ $\left.=7.9 \mathrm{~Hz},{ }^{5} \mathrm{~J}_{\mathrm{HH}}=1.2 \mathrm{~Hz}, 1 \mathrm{H}, \mathrm{C}_{\mathrm{Ar}} H\right), 7.32\left(\mathrm{td},{ }^{3} \mathrm{~J}_{\mathrm{HH}}=7.4 \mathrm{~Hz},{ }^{5} \mathrm{~J}_{\mathrm{HH}} 1.8 \mathrm{~Hz}, 1 \mathrm{H}, \mathrm{C}_{\mathrm{Ar}} H\right), 7.27\left(\mathrm{td},{ }^{3} \mathrm{~J}_{\mathrm{HH}}=7.6 \mathrm{~Hz},{ }^{5} \mathrm{~J}_{\mathrm{HH}}=1.4\right.$ $\left.\mathrm{Hz}, 1 \mathrm{H}, \mathrm{C}_{\mathrm{Ar}} H\right), 4.46\left(\mathrm{t},{ }^{3} \mathrm{~J}_{\mathrm{HH}}=8.5 \mathrm{~Hz}, 2 \mathrm{H}, \mathrm{CH}_{2}\right), 3.44\left(\mathrm{t},{ }^{3} \mathrm{~J}_{\mathrm{HH}}=8.5 \mathrm{~Hz}, 2 \mathrm{H}, \mathrm{CH}_{2}\right)$.

${ }^{13} \mathrm{C}-\left\{{ }^{1} \mathrm{H}\right\}$ NMR $\left(\mathbf{1 2 5 . 7} \mathbf{M H z}, \mathbf{C D C l}_{3}, \mathbf{2 9 8 K}\right): \delta(\mathrm{ppm})=166.2\left(\mathrm{~s}, \mathrm{C}^{\mathrm{IV}}\right), 133.1\left(\mathrm{~s}, \mathrm{C}^{\mathrm{IV}}\right), 132.5\left(\mathrm{~s}, \mathrm{C}^{\mathrm{IV}}\right), 131.1\left(\mathrm{~s}, C_{\mathrm{Ar}} \mathrm{H}\right)$, $130.8\left(\mathrm{~s}, C_{\mathrm{Ar}} \mathrm{H}\right), 130.6\left(\mathrm{~s}, C_{\mathrm{Ar}} \mathrm{H}\right), 126.8\left(\mathrm{~s}, C_{\mathrm{Ar}} \mathrm{H}\right), 65.3\left(\mathrm{~s}, C \mathrm{H}_{2}\right), 34.9\left(\mathrm{~s}, C \mathrm{H}_{2}\right)$.

2-(2-Bromophenyl)-4,5-dihydrothiazole, $4 \mathbf{m}$<smiles>Brc1ccccc1C1=NCCS1</smiles>

The general procedure yielded to the title compound as a colourless oil (234 mg, 98\%).

${ }^{1} \mathbf{H}$ NMR (400 MHz, $\mathbf{C D C l}_{\mathbf{3}}, \mathbf{2 9 8 K}$, TMS): $\delta(\mathrm{ppm})=7.60\left(\mathrm{dd},{ }^{3} \mathrm{~J}_{\mathrm{HH}}=8.0 \mathrm{~Hz},{ }^{5} \mathrm{~J}_{\mathrm{HH}}=1.1 \mathrm{~Hz}, 1 \mathrm{H}, \mathrm{C}_{\mathrm{Ar}} H\right), 7.52\left(\mathrm{dd},{ }^{3} \mathrm{~J}_{\mathrm{HH}}\right.$ $\left.=7.6 \mathrm{~Hz},{ }^{5} \mathrm{~J}_{\mathrm{HH}}=1.8 \mathrm{~Hz}, 1 \mathrm{H}, \mathrm{C}_{\mathrm{Ar}} H\right), 7.31\left(\mathrm{td},{ }^{3} \mathrm{~J}_{\mathrm{HH}}=7.6 \mathrm{~Hz},{ }^{5} \mathrm{~J}_{\mathrm{HH}}=1.2 \mathrm{~Hz}, 1 \mathrm{H}, \mathrm{C}_{\mathrm{Ar}} H\right), 7.22\left(\mathrm{td},{ }^{3} \mathrm{~J}_{\mathrm{HH}}=8.0 \mathrm{~Hz},{ }^{5} \mathrm{~J}_{\mathrm{HH}}=1.8\right.$ $\left.\mathrm{Hz}, 1 \mathrm{H}, \mathrm{C}_{\mathrm{Ar}} \mathrm{H}\right), 4.46\left(\mathrm{t},{ }^{3} \mathrm{~J}_{\mathrm{HH}}=8.5 \mathrm{~Hz}, 2 \mathrm{H}, \mathrm{CH}_{2}\right), 3.45\left(\mathrm{t},{ }^{3} \mathrm{~J}_{\mathrm{HH}}=8.5 \mathrm{~Hz}, 2 \mathrm{H}, \mathrm{CH}_{2}\right)$.

${ }^{13} \mathrm{C}-\left\{{ }^{1} \mathbf{H}\right\}$ NMR $\left(\mathbf{7 5} \mathbf{~ M H z}, \mathbf{C D C l}_{3}, \mathbf{2 9 8 K}\right): \delta(\mathrm{ppm})=167.2\left(\mathrm{~s}, \mathrm{C}^{\mathrm{IV}}\right), 135.2\left(\mathrm{~s}, \mathrm{C}^{\mathrm{IV}}\right), 133.7\left(\mathrm{~s}, C_{\mathrm{Ar}} \mathrm{H}\right), 131.1\left(\mathrm{~s}, C_{\mathrm{Ar}} \mathrm{H}\right)$, $130.5\left(\mathrm{~s}, C_{\mathrm{Ar}} \mathrm{H}\right), 127.3\left(\mathrm{~s}, C_{\mathrm{Ar}} \mathrm{H}\right), 121.2\left(\mathrm{~s}, \mathrm{C}^{\mathrm{IV}}\right), 65.4\left(\mathrm{~s}, \mathrm{CH}_{2}\right), 35.1\left(\mathrm{~s}, \mathrm{CH}_{2}\right)$.

HRMS calcd. for $\mathbf{C}_{9} \mathbf{H}_{9} B r N S(M+H)^{+} 241.9634$ found 241.9631.

2-(4,5-Dihydrothiazol-2-yl)aniline, $4 \mathbf{n}^{[1]}$<smiles>Nc1ccccc1C1=NCCS1</smiles>

The general procedure yielded to the title compound as a pale yellow oil (176 $\mathrm{mg}, 99 \%)$.

${ }^{1} \mathrm{H}$ NMR $\left(400 \mathrm{MHz}, \mathbf{C D C l}_{3}, \mathbf{2 9 8 K}\right.$, TMS): $\delta(\mathrm{ppm})=7.47\left(\mathrm{~d},{ }^{3} \mathrm{~J}_{\mathrm{HH}}=8.0 \mathrm{~Hz}, 1 \mathrm{H}, \mathrm{C}_{\mathrm{Ar}} H\right), 7.16\left(\mathrm{t},{ }^{3} \mathrm{~J}_{\mathrm{HH}}=7.5 \mathrm{~Hz}, 1 \mathrm{H}\right.$, $\left.\mathrm{C}_{\mathrm{Ar}} H\right), 6.68-6.64\left(\mathrm{~m}, 2 \mathrm{H}, \mathrm{C}_{\mathrm{Ar}} H\right), 6.17$ (s, $\left.2 \mathrm{H}, \mathrm{N} H_{2}\right), 4.49-4.45\left(\mathrm{~m}, 2 \mathrm{H}, \mathrm{CH}_{2}\right), 3.26-3.22\left(\mathrm{~m}, 2 \mathrm{H}, \mathrm{CH}_{2}\right)$.

${ }^{13} \mathrm{C}-\left\{{ }^{1} \mathrm{H}\right\}$ NMR (75 MHz, $\left.\mathbf{C D C l}_{3}, \mathbf{2 9 8 K}\right): \delta(\mathrm{ppm})=169.6\left(\mathrm{~s}, \mathrm{C}^{\mathrm{IV}}\right), 147.8\left(\mathrm{~s}, \mathrm{C}^{\mathrm{IV}}\right), 132.8\left(\mathrm{~s}, C_{\mathrm{Ar}} \mathrm{H}\right), 131.7\left(\mathrm{~s}, C_{\mathrm{Ar}} \mathrm{H}\right)$, $116.5\left(\mathrm{~s}, C_{\mathrm{Ar}} \mathrm{H}\right), 116.2\left(\mathrm{~s}, C_{\mathrm{Ar}} \mathrm{H}\right), 115.2\left(\mathrm{~s}, \mathrm{C}^{\mathrm{IV}}\right), 65.5\left(\mathrm{~s}, \mathrm{CH}_{2}\right), 32.1\left(\mathrm{~s}, \mathrm{CH}_{2}\right)$. 
2-(4-Chlorobenzyl)-4,5-dihydrothiazole, $\mathbf{4 o}^{[1]}$<smiles>Clc1ccc(CC2=NCCS2)cc1</smiles>

The general procedure yielded to the title compound as a yellow oil (194 mg, 92\%).

${ }^{1} \mathbf{H}$ NMR (400 MHz, CDCl 3 , 298K, TMS): $\delta(\mathrm{ppm})=7.28\left(\mathrm{~d},{ }^{3} \mathrm{~J}_{\mathrm{HH}}=8.4 \mathrm{~Hz}, 2 \mathrm{H}, \mathrm{C}_{\mathrm{Ar}} H\right), 7.21\left(\mathrm{~d},{ }^{3} \mathrm{~J}_{\mathrm{HH}}=8.4 \mathrm{~Hz}, 2 \mathrm{H}\right.$, $\left.\mathrm{C}_{\mathrm{Ar}} \mathrm{H}\right), 4.23\left(\mathrm{t},{ }^{3} \mathrm{~J}_{\mathrm{HH}}=8.4 \mathrm{HZ}, 2 \mathrm{H}, \mathrm{CH}_{2}\right), 3.77\left(\mathrm{~s}, 2 \mathrm{H}, \mathrm{CH}_{2}\right), 3.26\left(\mathrm{t},{ }^{3} \mathrm{~J}_{\mathrm{HH}}=8.4 \mathrm{~Hz}, 2 \mathrm{H}, \mathrm{CH}_{2}\right)$.

${ }^{13} \mathrm{C}-\left\{{ }^{1} \mathrm{H}\right\}$ NMR (75 MHz, CDCl $\left.3, \mathbf{2 9 8 K}\right): \delta(\mathrm{ppm})=170.1\left(\mathrm{~s}, \mathrm{C}^{\mathrm{IV}}\right), 134.8\left(\mathrm{~s}, \mathrm{C}^{\mathrm{IV}}\right), 133.3\left(\mathrm{~s}, \mathrm{C}^{\mathrm{IV}}\right), 130.7\left(\mathrm{~s}, C_{\mathrm{Ar}} \mathrm{H}\right), 129.0$ (s, $\left.C_{\mathrm{Ar}} \mathrm{H}\right), 64.9\left(\mathrm{~s}, \mathrm{CH}_{2}\right), 40.3\left(\mathrm{~s}, \mathrm{CH}_{2}\right), 34.5\left(\mathrm{~s}, \mathrm{CH}_{2}\right)$.

2-Benzyl-4,5-dihydrothiazole, $\mathbf{4} \mathbf{p}^{[1]}$<smiles>c1ccc(CC2=NCCS2)cc1</smiles>

The general procedure yielded to the title compound as a yellow oil (172 mg, 97\%).

${ }^{1} \mathrm{H}$ NMR (400 MHz, $\mathbf{C D C l}_{3}, \mathbf{2 9 8 K}$, TMS): $\delta(\mathrm{ppm})=7.30-7.19\left(\mathrm{~m}, 5 \mathrm{H}, \mathrm{C}_{\mathrm{Ar}} \mathrm{H}\right), 4.19-4.14\left(\mathrm{~m}, 2 \mathrm{H}, \mathrm{CH}_{2}\right), 3.78(\mathrm{~s}, 2 \mathrm{H}$, $\left.\mathrm{CH}_{2}\right), 3.18-3.13\left(\mathrm{~m}, 2 \mathrm{H}, \mathrm{CH}_{2}\right)$.

${ }^{13} \mathrm{C}-\left\{{ }^{1} \mathbf{H}\right\}$ NMR $\left(75 \mathbf{~ M H z}, \mathbf{C D C l}_{3}, \mathbf{2 9 8 K}\right): \delta(\mathrm{ppm})=170.1\left(\mathrm{~s}, \mathrm{C}^{\mathrm{IV}}\right), 136.1\left(\mathrm{~s}, \mathrm{C}^{\mathrm{IV}}\right), 129.1\left(\mathrm{~s}, C_{\mathrm{Ar}} \mathrm{H}\right), 128.5\left(\mathrm{~s}, C_{\mathrm{Ar}} \mathrm{H}\right)$, $127.0\left(\mathrm{~s}, C_{\mathrm{Ar}} \mathrm{H}\right), 64.6\left(\mathrm{~s}, \mathrm{CH}_{2}\right), 40.7\left(\mathrm{~s}, \mathrm{CH}_{2}\right), 34.1\left(\mathrm{~s}, \mathrm{CH}_{2}\right)$.

2-(2-Bromobenzyl)-4,5-dihydrothiazole, $4 \mathbf{q}$<smiles>Brc1ccccc1CC1=NCCS1</smiles>

The general procedure yielded to the title compound as a colourless oil (232 mg, 91\%).

${ }^{1} \mathrm{H}$ NMR (500 MHz, CDCl 3 , 298K, TMS): $\delta(\mathrm{ppm})=7.54\left(\mathrm{~d},{ }^{3} \mathrm{~J}_{\mathrm{HH}}=7.5 \mathrm{~Hz}, 1 \mathrm{H}, \mathrm{C}_{\mathrm{Ar}} H\right), 7.32-7.30\left(\mathrm{~m}, 1 \mathrm{H}, \mathrm{C}_{\mathrm{Ar}} H\right)$, 7.26-7.23 (m, $\left.1 \mathrm{H}, \mathrm{C}_{\mathrm{Ar}} H\right), 7.12-7.08\left(\mathrm{~m}, 1 \mathrm{H}, \mathrm{C}_{\mathrm{Ar}} H\right), 4.21\left(\mathrm{t},{ }^{3} \mathrm{~J}_{\mathrm{HH}}=8.3 \mathrm{~Hz}, 2 \mathrm{H}, \mathrm{CH}_{2}\right), 3.95\left(\mathrm{~s}, 2 \mathrm{H}, \mathrm{CH}_{2}\right), 3.24\left(\mathrm{t},{ }^{3} \mathrm{~J}_{\mathrm{HH}}=\right.$ $\left.8.3 \mathrm{~Hz}, 2 \mathrm{H}, \mathrm{CH}_{2}\right)$.

${ }^{13} \mathrm{C}-\left\{{ }^{1} \mathrm{H}\right\}$ NMR $\left(\mathbf{7 5} \mathbf{~ M H z}, \mathbf{C D C l}_{3}, \mathbf{2 9 8 K}\right): \delta(\mathrm{ppm})=169.0\left(\mathrm{~s}, \mathrm{C}^{\mathrm{IV}}\right), 136.2\left(\mathrm{~s}, \mathrm{C}^{\mathrm{IV}}\right), 133.0\left(\mathrm{~s}, C_{\mathrm{Ar}} \mathrm{H}\right), 131.3\left(\mathrm{~s}, C_{\mathrm{Ar}} \mathrm{H}\right)$, $128.9\left(\mathrm{~s}, C_{\mathrm{Ar}} \mathrm{H}\right), 127.7\left(\mathrm{~s}, C_{\mathrm{Ar}} \mathrm{H}\right), 125.0\left(\mathrm{~s}, \mathrm{C}^{\mathrm{IV}}\right), 64.8\left(\mathrm{~s}, \mathrm{CH}_{2}\right), 40.8\left(\mathrm{~s}, \mathrm{CH}_{2}\right), 34.2\left(\mathrm{~s}, \mathrm{CH}_{2}\right)$.

HRMS calcd. for $\mathbf{C}_{10} \mathbf{H}_{11} B r N S(\mathbf{M}+\mathbf{H})^{+} 255.9790$ found 255.9790 .

2-(Pyridin-2-yl)-4,5-dihydrothiazole, $4 \mathbf{r}^{[1]}$<smiles>c1ccc(C2=NCCS2)nc1</smiles>

The general procedure yielded to the title compound as a colourless solid (159 mg, 97\%). 
${ }^{1}$ H NMR (400 MHz, CDCl3, 298K, TMS): $\delta(\mathrm{ppm})=8.66\left(\mathrm{~s}, 1 \mathrm{H}, \mathrm{C}_{\mathrm{Ar}} H\right), 8.08-8.05\left(\mathrm{~m}, 1 \mathrm{H}, \mathrm{C}_{\mathrm{Ar}} H\right), 7.79-7.73(\mathrm{~m}, 1 \mathrm{H}$, $\left.\mathrm{C}_{\mathrm{Ar}} H\right), 7.48-7.33\left(\mathrm{~m}, 1 \mathrm{H}, \mathrm{C}_{\mathrm{Ar}} H\right), 4.57-4.51\left(\mathrm{~m}, 2 \mathrm{H}, \mathrm{CH}_{2}\right), 3.41-3.34\left(\mathrm{~m}, 2 \mathrm{H}, \mathrm{CH}_{2}\right)$.

${ }^{13} \mathrm{C}-\left\{{ }^{1} \mathrm{H}\right\}$ NMR (75 MHz, CDCl3, 298K): $\delta(\mathrm{ppm})=171.1\left(\mathrm{~s}, \mathrm{C}^{\mathrm{IV}}\right), 151.3\left(\mathrm{~s}, \mathrm{C}^{\mathrm{IV}}\right), 149.4\left(\mathrm{~s}, C_{\mathrm{Ar}} \mathrm{H}\right), 136.7\left(\mathrm{~s}, C_{\mathrm{Ar}} \mathrm{H}\right)$, $125.5\left(\mathrm{~s}, C_{\mathrm{Ar}} \mathrm{H}\right), 121.6\left(\mathrm{~s}, C_{\mathrm{Ar}} \mathrm{H}\right), 65.9\left(\mathrm{~s}, \mathrm{CH}_{2}\right), 32.7\left(\mathrm{~s}, \mathrm{CH}_{2}\right)$.

Melting point: $92^{\circ} \mathrm{C}$

2-(Pyridin-3-yl)-4,5-dihydrothiazole, $4 \mathbf{s}^{[1]}$<smiles>c1cncc(C2=NCCS2)c1</smiles>

The general procedure yielded to the title compound as a yellow oil (158 mg, 96\%).

${ }^{1} \mathbf{H}$ NMR (400 MHz, $\mathbf{C D C l}_{3}, \mathbf{2 9 8 K}$, TMS): $\delta(\mathrm{ppm})=9.04\left(\mathrm{~d},{ }^{5} \mathrm{~J}_{\mathrm{HH}} 1.6 \mathrm{~Hz}, 1 \mathrm{H}, \mathrm{C}_{\mathrm{Ar}} H\right), 8.68\left(\mathrm{dd},{ }^{3} \mathrm{~J}_{\mathrm{HH}}=4.8 \mathrm{~Hz},{ }^{5} \mathrm{~J}_{\mathrm{HH}}=\right.$ $\left.1.6 \mathrm{~Hz}, 1 \mathrm{H}, \mathrm{C}_{\mathrm{Ar}} H\right), 8.10\left(\mathrm{dt},{ }^{3} \mathrm{~J}_{\mathrm{HH}}=8.0 \mathrm{~Hz},{ }^{5} \mathrm{~J}_{\mathrm{HH}}=1.9 \mathrm{~Hz}, 1 \mathrm{H}, \mathrm{C}_{\mathrm{Ar}} H\right), 7.35\left(\mathrm{dd},{ }^{3} \mathrm{~J}_{\mathrm{HH}}=8.0 \mathrm{~Hz},{ }^{5} \mathrm{~J}_{\mathrm{HH}}=4.8 \mathrm{~Hz}, 1 \mathrm{H}, \mathrm{C}_{\mathrm{Ar}} H\right)$, $4.46\left(\mathrm{t},{ }^{3} \mathrm{~J}_{\mathrm{HH}}=8.4 \mathrm{~Hz}, 2 \mathrm{H}, \mathrm{CH}_{2}\right), 3.45\left(\mathrm{t},{ }^{3} \mathrm{~J}_{\mathrm{HH}}=8.4 \mathrm{~Hz}, 2 \mathrm{H}, \mathrm{CH}_{2}\right)$.

${ }^{13} \mathbf{C}-\left\{{ }^{1} \mathbf{H}\right\}$ NMR $\left(\mathbf{7 5} \mathbf{~ M H z}, \mathbf{C D C l}_{3}, \mathbf{2 9 8 K}\right): \delta(\mathrm{ppm})=165.6\left(\mathrm{~s}, \mathrm{C}^{\mathrm{IV}}\right), 151.8\left(\mathrm{~s}, C_{\mathrm{Ar}} \mathrm{H}\right), 149.4\left(\mathrm{~s}, C_{\mathrm{Ar}} \mathrm{H}\right), 135.4\left(\mathrm{~s}, C_{\mathrm{Ar}} \mathrm{H}\right)$, $129.1\left(\mathrm{~s}, \mathrm{C}^{\mathrm{IV}}\right), 123.3\left(\mathrm{~s}, \mathrm{C}_{\mathrm{Ar}} \mathrm{H}\right), 65.2\left(\mathrm{~s}, \mathrm{CH}_{2}\right), 33.9\left(\mathrm{~s}, \mathrm{CH}_{2}\right)$.

2-(Pyridin-4-yl)-4,5-dihydrothiazole, $\mathbf{4 t}^{[1]}$<smiles>c1cc(C2=NCCS2)ccn1</smiles>

The general procedure yielded to the title compound as a colourless solid (157 mg, 96\%).

${ }^{1} \mathbf{H}$ NMR (400 MHz, CDCl 3 , 298K, TMS): $\delta(\mathrm{ppm})=8.70\left(\mathrm{dd},{ }^{3} \mathrm{~J}_{\mathrm{HH}}=4.5 \mathrm{~Hz},{ }^{5} \mathrm{~J}_{\mathrm{HH}}=1.7 \mathrm{~Hz}, 2 \mathrm{H}, \mathrm{C}_{\mathrm{Ar}} H\right), 7.67\left(\mathrm{dd},{ }^{3} \mathrm{~J}_{\mathrm{HH}}\right.$ $\left.=4.5 \mathrm{~Hz},{ }^{5} \mathrm{~J}_{\mathrm{HH}}=1.7 \mathrm{~Hz}, 2 \mathrm{H}, \mathrm{C}_{\mathrm{Ar}} H\right), 4.50\left(\mathrm{t},{ }^{3} \mathrm{~J}_{\mathrm{HH}}=8.5 \mathrm{~Hz}, 2 \mathrm{H}, \mathrm{CH}_{2}\right), 3.47\left(\mathrm{t},{ }^{3} \mathrm{~J}_{\mathrm{HH}}=8.5 \mathrm{~Hz}, 2 \mathrm{H}, \mathrm{CH}_{2}\right)$.

${ }^{13} \mathrm{C}-\left\{{ }^{1} \mathrm{H}\right\}$ NMR $\left(75 \mathrm{MHz}, \mathrm{CDCl}_{3}, \mathbf{2 9 8 K}\right): \delta(\mathrm{ppm})=167.2\left(\mathrm{~s}, \mathrm{C}^{\mathrm{IV}}\right), 150.6\left(\mathrm{~s}, C_{\mathrm{Ar}} \mathrm{H}\right), 140.3\left(\mathrm{~s}, \mathrm{C}^{\mathrm{IV}}\right), 122.3\left(\mathrm{~s}, C_{\mathrm{Ar}} \mathrm{H}\right)$, $65.7\left(\mathrm{~s}, \mathrm{CH}_{2}\right), 34.2\left(\mathrm{~s}, \mathrm{CH}_{2}\right)$.

Melting point: $73^{\circ} \mathrm{C}$

2-(1H-Pyrrol-2-yl)-4,5-dihydrothiazole, $4 \mathbf{u}^{[7]}$<smiles>c1c[nH]c(C2=NCCS2)c1</smiles>

The general procedure yielded to the title compound as a colourless solid (300 mg, 98\%).

${ }^{1} \mathrm{H}$ NMR (400 MHz, CDCl 3 , 298K, TMS): $\delta(\mathrm{ppm})=10.54(\mathrm{~s}, 1 \mathrm{H}, \mathrm{NH}), 6.90\left(\mathrm{~s}, 1 \mathrm{H}, \mathrm{C}_{\mathrm{Ar}} H\right), 6.64\left(\mathrm{~d},{ }^{3} \mathrm{~J}_{\mathrm{HH}}=2.9 \mathrm{~Hz}\right.$, $\left.1 \mathrm{H}, \mathrm{C}_{\mathrm{Ar}} H\right), 6.23\left(\mathrm{t},{ }^{3} \mathrm{~J}_{\mathrm{HH}}=2.9 \mathrm{~Hz}, 1 \mathrm{H}, \mathrm{C}_{\mathrm{Ar}} H\right), 4.35\left(\mathrm{t},{ }^{3} \mathrm{~J}_{\mathrm{HH}}=8.1 \mathrm{~Hz}, 2 \mathrm{H}, \mathrm{CH}_{2}\right), 3.38\left(\mathrm{t},{ }^{3} \mathrm{~J}_{\mathrm{HH}}=8.1 \mathrm{~Hz}, 2 \mathrm{H}, \mathrm{CH}_{2}\right)$.

${ }^{13} \mathrm{C}-\left\{{ }^{1} \mathrm{H}\right\}$ NMR $\left(75 \mathrm{MHz}, \mathbf{C D C l}_{3}, \mathbf{2 9 8 K}\right): \delta(\mathrm{ppm})=161.5\left(\mathrm{~s}, \mathrm{C}^{\mathrm{IV}}\right), 126.0\left(\mathrm{~s}, \mathrm{C}^{\mathrm{IV}}\right), 122.6\left(\mathrm{~s}, C_{\mathrm{Ar}} \mathrm{H}\right), 115.0\left(\mathrm{~s}, C_{\mathrm{Ar}} \mathrm{H}\right)$, $110.0\left(\mathrm{~s}, C_{\mathrm{Ar}} \mathrm{H}\right), 64.1\left(\mathrm{~s}, \mathrm{CH}_{2}\right), 33.7\left(\mathrm{~s}, \mathrm{CH}_{2}\right)$. 
2-(2-Furanyl)-4,5-dihydrothiazole, $\mathbf{4} \mathbf{v}^{[6]}$<smiles>c1coc(C2=NCCS2)c1</smiles>

The general procedure yielded to the title compound as a yellow oil (291 mg, 95\%).

${ }^{1} \mathrm{H}$ NMR (400 MHz, $\mathbf{C D C l}_{3}, \mathbf{2 9 8 K}$, TMS): $\delta(\mathrm{ppm})=7.52-7.51\left(\mathrm{~m}, 1 \mathrm{H}, \mathrm{C}_{\mathrm{Ar}} H\right), 6.88\left(\mathrm{~d},{ }^{3} \mathrm{~J}_{\mathrm{HH}}=3.5 \mathrm{~Hz}, 1 \mathrm{H}, \mathrm{C}_{\mathrm{Ar}} H\right), 6.46$ $\left(\mathrm{dd},{ }^{3} \mathrm{~J}_{\mathrm{HH}}=3.5 \mathrm{~Hz},{ }^{5} \mathrm{~J}_{\mathrm{HH}} 1.8 \mathrm{~Hz}, 1 \mathrm{H}, \mathrm{C}_{\mathrm{Ar}} H\right), 4.37\left(\mathrm{t},{ }^{3} \mathrm{~J}_{\mathrm{HH}}=8.3 \mathrm{~Hz}, 2 \mathrm{H}, \mathrm{CH}_{2}\right), 3.34\left(\mathrm{t},{ }^{3} \mathrm{~J}_{\mathrm{HH}}=8.3 \mathrm{~Hz}, 2 \mathrm{H}, \mathrm{CH}_{2}\right)$.

${ }^{13} \mathrm{C}-\left\{{ }^{1} \mathrm{H}\right\}$ NMR $\left(\mathbf{7 5} \mathbf{~ M H z}, \mathbf{C D C l}_{3}, \mathbf{2 9 8 K}\right): \delta(\mathrm{ppm})=157.2\left(\mathrm{~s}, \mathrm{C}^{\mathrm{IV}}\right), 147.6\left(\mathrm{~s}, \mathrm{C}^{\mathrm{IV}}\right), 144.4\left(\mathrm{~s}, C_{\mathrm{Ar}} \mathrm{H}\right), 113.2\left(C_{\mathrm{Ar}} \mathrm{H}\right), 111.4$ $\left(C_{\mathrm{Ar}} \mathrm{H}\right), 64.5\left(\mathrm{~s}, \mathrm{CH}_{2}\right), 33.1$ (s, $\left.\mathrm{CH}_{2}\right)$.

2-(Pent-4-yn-1-yl)-4,5-dihydrothiazole, $4 \mathbf{w}$

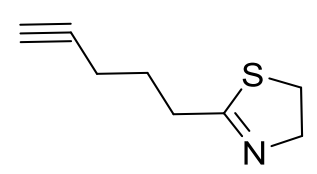

The general procedure yielded to the title compound as a colourless oil (132 $\mathrm{mg}, 87 \%)$.

${ }^{1} \mathrm{H}$ NMR (400 MHz, $\left.\mathbf{C D C l}_{3}, \mathbf{2 9 8 K}, \mathbf{T M S}\right): \delta(\mathrm{ppm})=4.24-4.19\left(\mathrm{~m}, 2 \mathrm{H}, \mathrm{CH}_{2}\right), 3.31-3.21\left(\mathrm{~m}, 2 \mathrm{H}, \mathrm{CH}_{2}\right), 2.65-2.61(\mathrm{~m}$, $\left.2 \mathrm{H}, \mathrm{CH}_{2}\right), 2.31-2.27\left(\mathrm{~m}, 2 \mathrm{H}, \mathrm{CH}_{2}\right), 2.00-1.93(\mathrm{~m}, 1 \mathrm{H}, \mathrm{CH}), 1.93-1.86\left(\mathrm{~m}, 2 \mathrm{H}, \mathrm{CH}_{2}\right)$.

${ }^{13} \mathrm{C}-\left\{{ }^{1} \mathbf{H}\right\}$ NMR $\left(\mathbf{7 5} \mathbf{~ M H z}, \mathbf{C D C l}\right.$, 298K): $\delta(\mathrm{ppm})=170.8\left(\mathrm{~s}, \mathrm{C}^{\mathrm{IV}}\right), 83.6(\mathrm{~s}, \mathrm{CH}), 69.3\left(\mathrm{~s}, \mathrm{C}^{\mathrm{IV}}\right), 64.8\left(\mathrm{~s}, \mathrm{CH}_{2}\right), 34.0(\mathrm{~s}$, $\left.\mathrm{CH}_{2}\right), 33.2\left(\mathrm{~s}, \mathrm{CH}_{2}\right), 26.3\left(\mathrm{~s}, \mathrm{CH}_{2}\right), 18.1\left(\mathrm{~s}, \mathrm{CH}_{2}\right)$.

HRMS calcd. for $\mathrm{C}_{8} \mathrm{H}_{12} \mathrm{NS}(\mathrm{M}+\mathrm{H})^{+} 154.0685$ found 154.0683 .

2-Pentyl-4,5-dihydrothiazole, $\mathbf{4} \mathbf{x}^{[8]}$

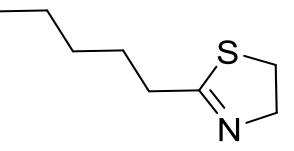

The general procedure yielded after flash chromatography $\left(\mathrm{SiO}_{2}\right.$, penthane/ ethyl acetate 7:3) to the title compound as a colourless oil ( $85 \mathrm{mg}, 55 \%)$.

${ }^{1} \mathbf{H}$ NMR (400 MHz, CDCl, $\mathbf{2 9 8 K}$, TMS): $\delta(\mathrm{ppm})=4.21\left(\mathrm{tt},{ }^{3} \mathrm{~J}_{\mathrm{HH}}=8.4 \mathrm{~Hz},{ }^{5} \mathrm{~J}_{\mathrm{HH}}=1.4 \mathrm{~Hz}, 2 \mathrm{H}, \mathrm{CH}_{2}\right), 3.27\left(\mathrm{t},{ }^{3} \mathrm{~J}_{\mathrm{HH}}=\right.$ $\left.8.4 \mathrm{~Hz}, 2 \mathrm{H}, \mathrm{CH}_{2}\right), 2.52-2.48\left(\mathrm{~m}, 2 \mathrm{H}, \mathrm{CH}_{2}\right), 1.69-1.61\left(\mathrm{~m}, 2 \mathrm{H}, \mathrm{CH}_{2}\right), 1.36-1.31\left(\mathrm{~m}, 4 \mathrm{H}, \mathrm{CH}_{2}\right), 0.92-0.88\left(\mathrm{~m}, 3 \mathrm{H}, \mathrm{CH}_{3}\right)$.

${ }^{13} \mathrm{C}-\left\{{ }^{1} \mathrm{H}\right\}$ NMR $\left(\mathbf{7 5} \mathbf{~ M H z}, \mathbf{C D C l}_{3}, \mathbf{2 9 8 K}\right): \delta(\mathrm{ppm})=172.0\left(\mathrm{~s}, \mathrm{C}^{\mathrm{IV}}\right), 64.8\left(\mathrm{~s}, \mathrm{CH}_{2}\right), 34.6\left(\mathrm{~s}, \mathrm{CH}_{2}\right), 34.0\left(\mathrm{~s}, \mathrm{CH}_{2}\right), 31.6(\mathrm{~s}$, $\left.\mathrm{CH}_{2}\right), 27.5\left(\mathrm{~s}, \mathrm{CH}_{2}\right), 22.6\left(\mathrm{~s}, \mathrm{CH}_{2}\right), 14.2\left(\mathrm{~s}, \mathrm{CH}_{3}\right)$.

HRMS calcd. for $\mathbf{C}_{8} \mathbf{H}_{16} \mathbf{N S}(\mathbf{M}+\mathbf{H})^{+} 158.0998$ found 158.0997 . 
2-Cyclohexyl-4,5-dihydrothiazole, $\mathbf{4 y}{ }^{[8]}$

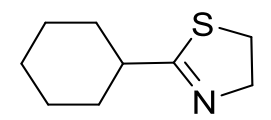

The general procedure yielded after flash chromatography $\left(\mathrm{SiO}_{2}\right.$, penthane/ ethyl acetate 8:2) to the title compound as a colourless oil ( $77 \mathrm{mg}, 45 \%)$.

${ }^{1} \mathbf{H}$ NMR (400 MHz, CDCl $\left., \mathbf{2 9 8 K}, \mathbf{T M S}\right): \delta(\mathrm{ppm})=4.20\left(\mathrm{td},{ }^{3} \mathrm{~J}_{\mathrm{HH}}=8.4 \mathrm{~Hz},{ }^{5} \mathrm{~J}_{\mathrm{HH}}=1.1 \mathrm{~Hz}, 2 \mathrm{H}, \mathrm{CH}_{2}\right), 3.22\left(\mathrm{t},{ }^{3} \mathrm{~J}_{\mathrm{HH}}=\right.$ $\left.8.4 \mathrm{~Hz}, 2 \mathrm{H}, \mathrm{CH}_{2}\right), 2.53-2.46(\mathrm{~m}, 1 \mathrm{H}, \mathrm{CH}), 1.97-1.93\left(\mathrm{~m}, 2 \mathrm{H}, \mathrm{CH}_{2}\right), 1.81-1.76\left(\mathrm{~m}, 2 \mathrm{H}, \mathrm{CH}_{2}\right), 1.70-1.65\left(\mathrm{~m}, 1 \mathrm{H}, \mathrm{CH}_{2}\right)$, $1.57-1.16\left(\mathrm{~m}, 5 \mathrm{H}, \mathrm{CH}_{2}\right)$.

${ }^{13} \mathrm{C}-\left\{{ }^{1} \mathrm{H}\right\}$ NMR (75 MHz, CDCl $\left.3,298 K\right): \delta(\mathrm{ppm})=176.7\left(\mathrm{~s}, \mathrm{C}^{\mathrm{IV}}\right), 64.7\left(\mathrm{~s}, \mathrm{CH}_{2}\right), 43.6(\mathrm{~s}, \mathrm{CH}), 33.3\left(\mathrm{~s}, \mathrm{CH}_{2}\right), 31.8(\mathrm{~s}$, $\left.\mathrm{CH}_{2}\right), 26.2\left(\mathrm{~s}, \mathrm{CH}_{2}\right)$. 


\section{$5 \quad$ NMR spectra}

${ }^{1} \mathrm{H}$ NMR (500 MHz, $\left.\mathrm{CDCl}_{3}, 298 \mathrm{~K}, \mathrm{TMS}\right)$ of 2-phenyl-4,5-dihydrothiazole, $4 \mathbf{a}$
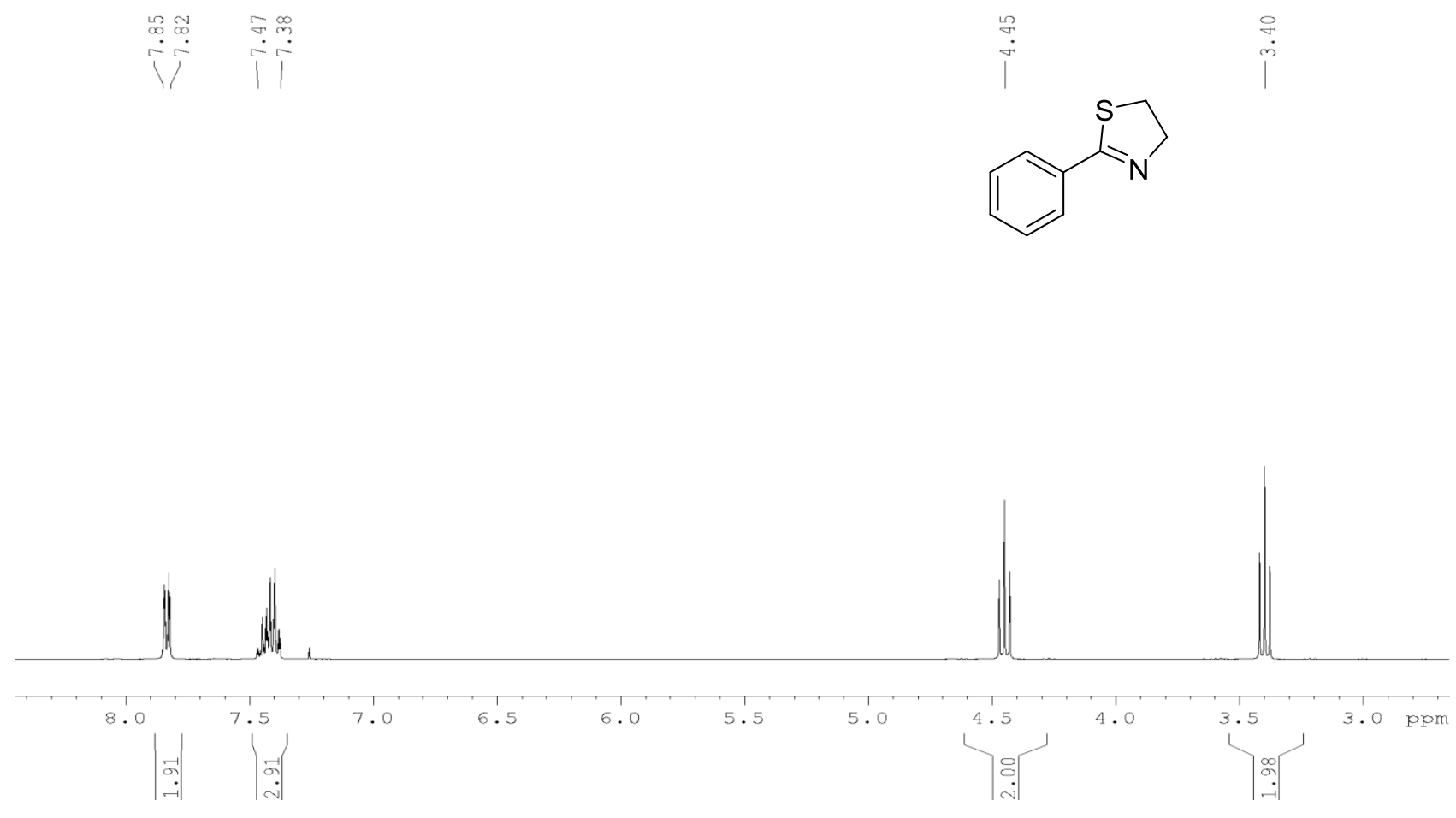

${ }^{13} \mathrm{C}-\left\{{ }^{1} \mathrm{H}\right\}$ NMR $\left(125.7 \mathrm{MHz}, \mathrm{CDCl}_{3}, 298 \mathrm{~K}\right)$ of 2-phenyl-4,5-dihydrothiazole, $4 \mathbf{a}$
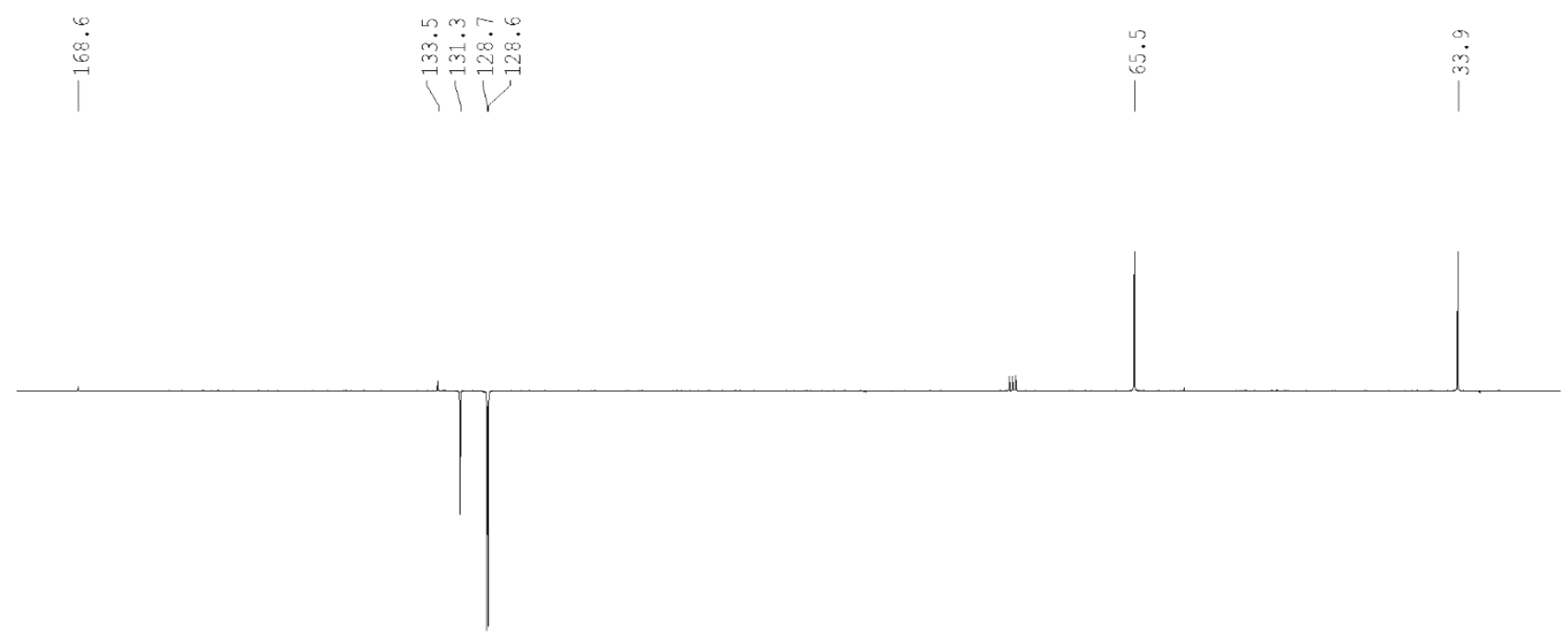

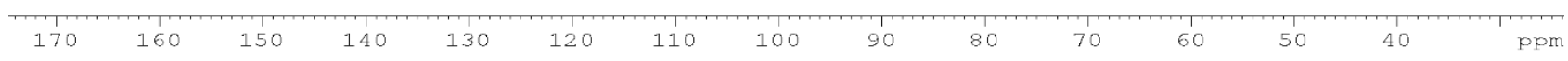


${ }^{1} \mathrm{H}$ NMR (400 MHz, $\left.\mathrm{CDCl}_{3}, 298 \mathrm{~K}, \mathrm{TMS}\right)$ of 2-(4-fluorophenyl)-4,5-dihydrothiazole, 4b
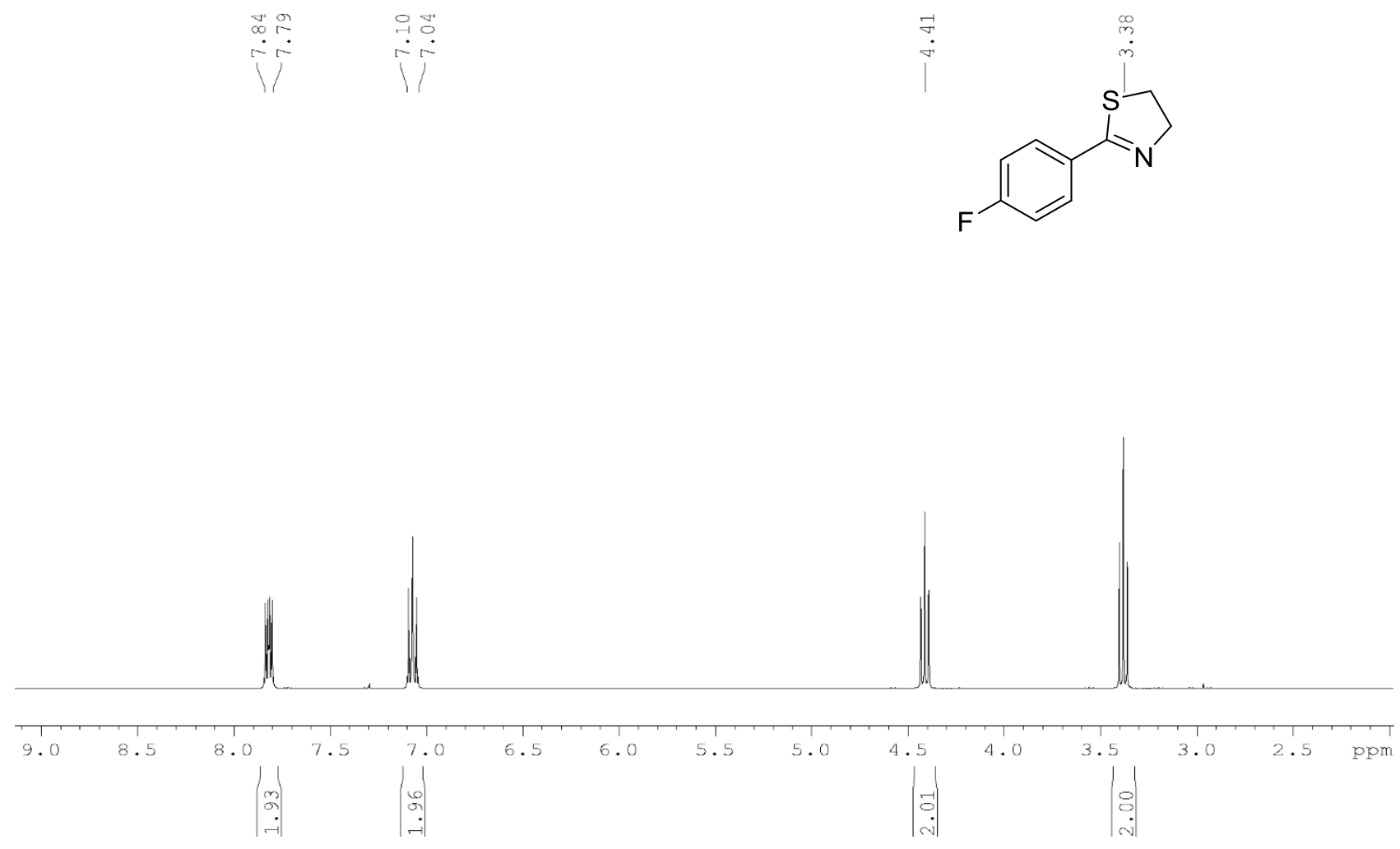

${ }^{13} \mathrm{C}-\left\{{ }^{1} \mathrm{H}\right\}$ NMR $\left(75 \mathrm{MHz}, \mathrm{CDCl}_{3}, 298 \mathrm{~K}\right)$ of 2-(4-fluorophenyl)-4,5-dihydrothiazole, $4 \mathbf{b}$
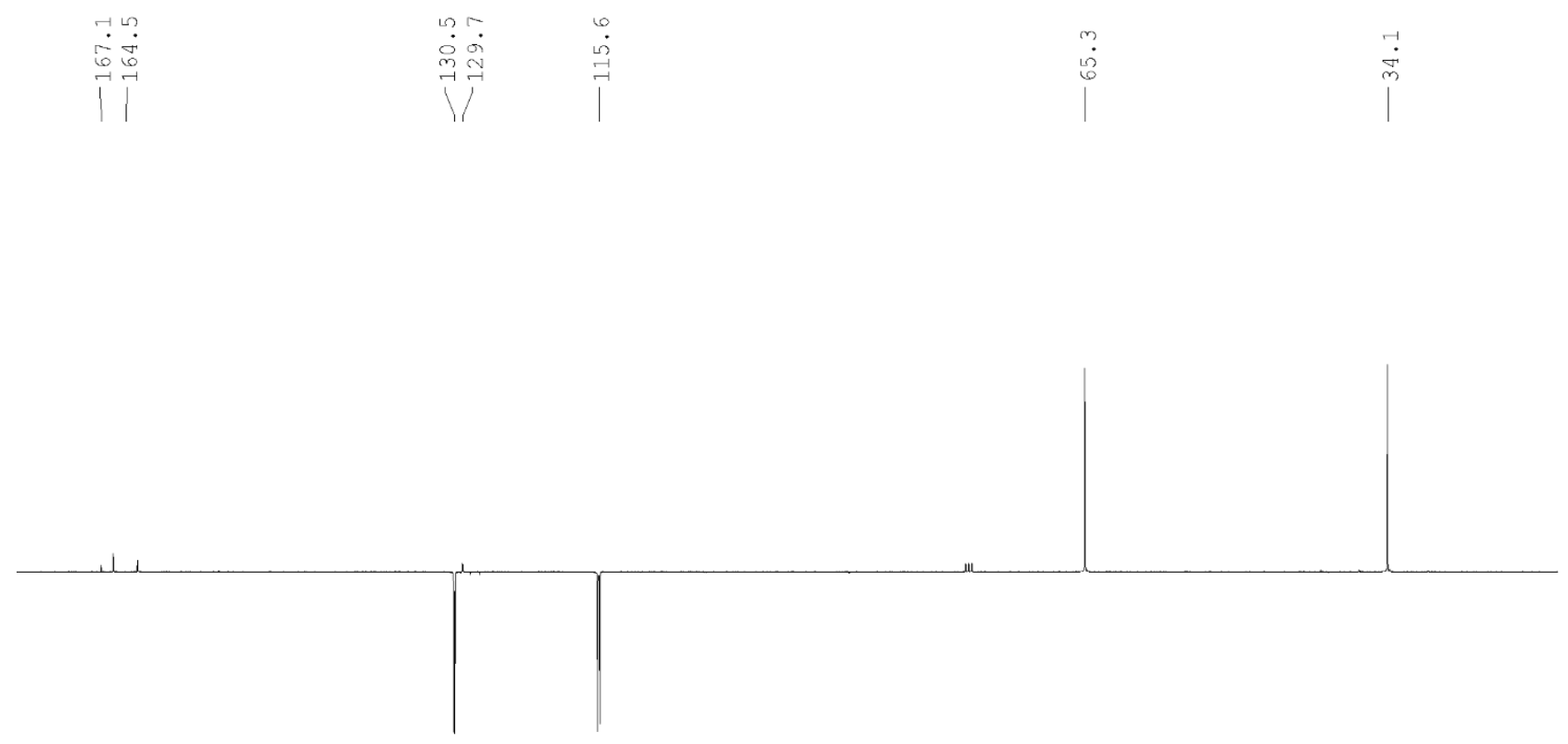

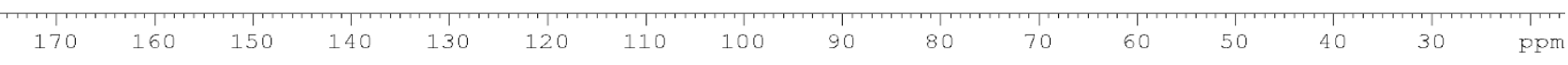


${ }^{19} \mathrm{~F}-\left\{{ }^{1} \mathrm{H}\right\}$ NMR (376 MHz, 298K) of 2-(4-fluorophenyl)-4,5-dihydrothiazole, 4b

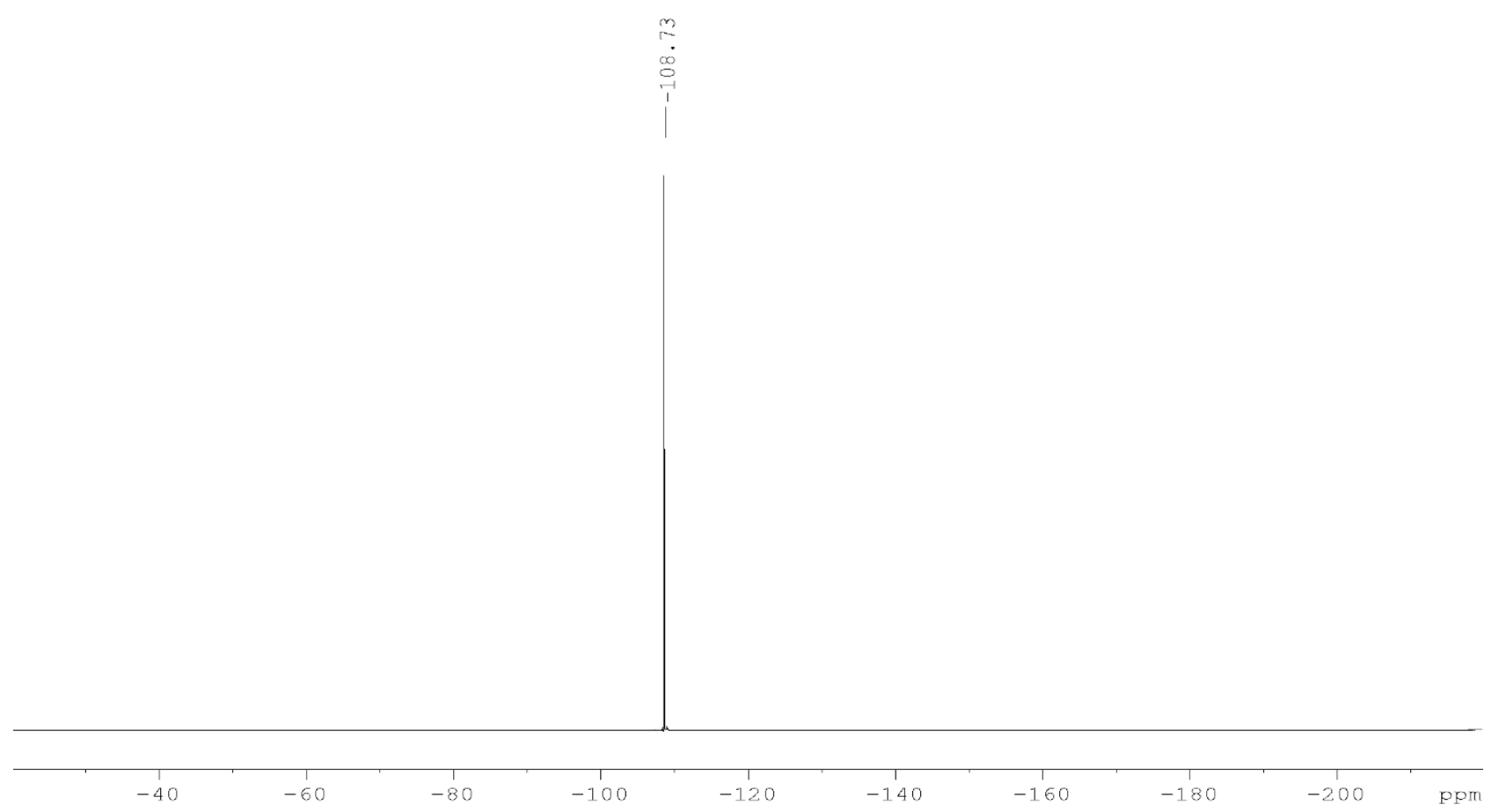


${ }^{1} \mathrm{H}$ NMR (400 MHz, CDCl3, 298K, TMS) of 2-(4-chlorophenyl)-4,5-dihydrothiazole, 4c
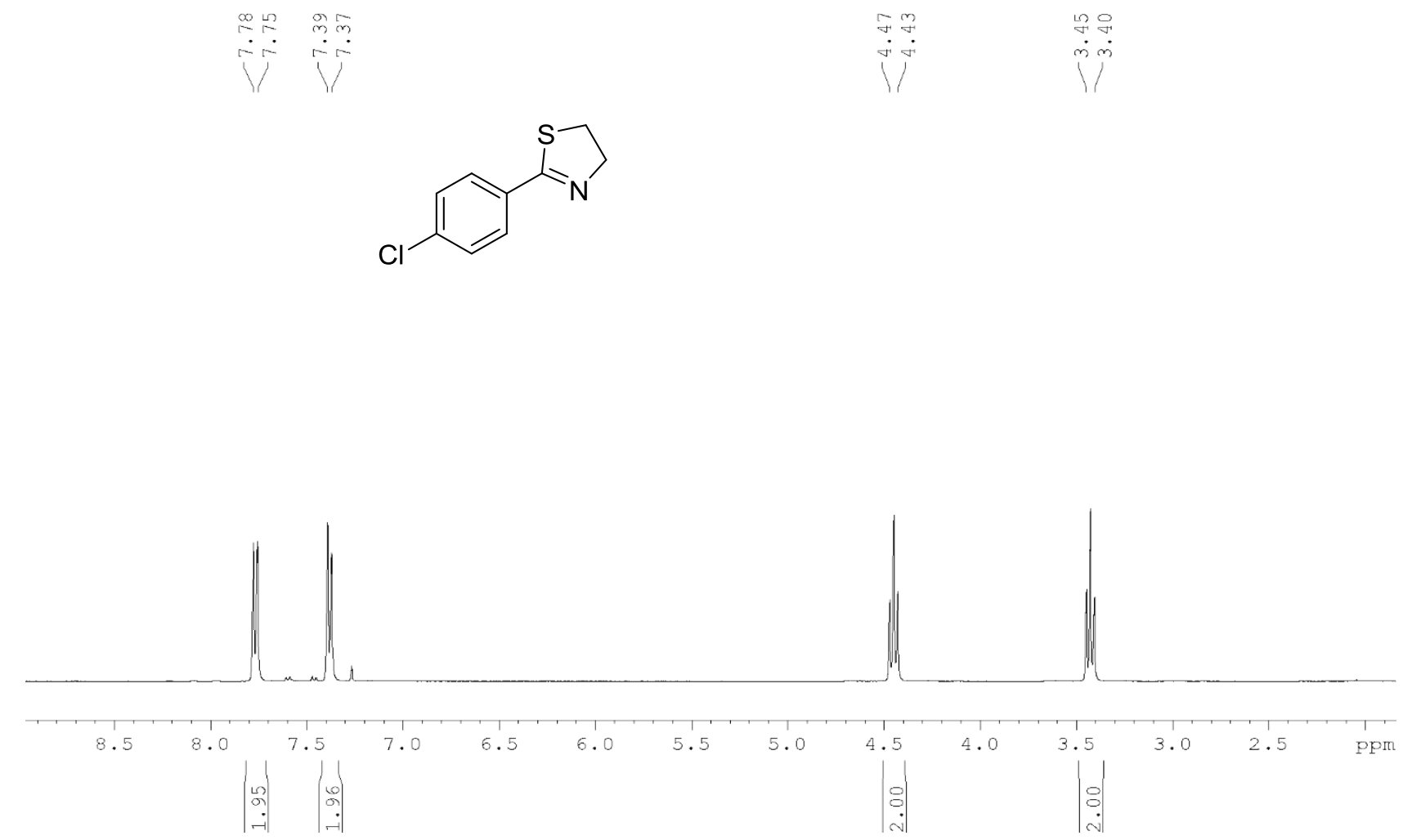

${ }^{13} \mathrm{C}-\left\{{ }^{1} \mathrm{H}\right\}$ NMR $\left(75 \mathrm{MHz}, \mathrm{CDCl}_{3}, 298 \mathrm{~K}\right)$ of 2-(4-chlorophenyl)-4,5-dihydrothiazole, $\mathbf{4 c}$
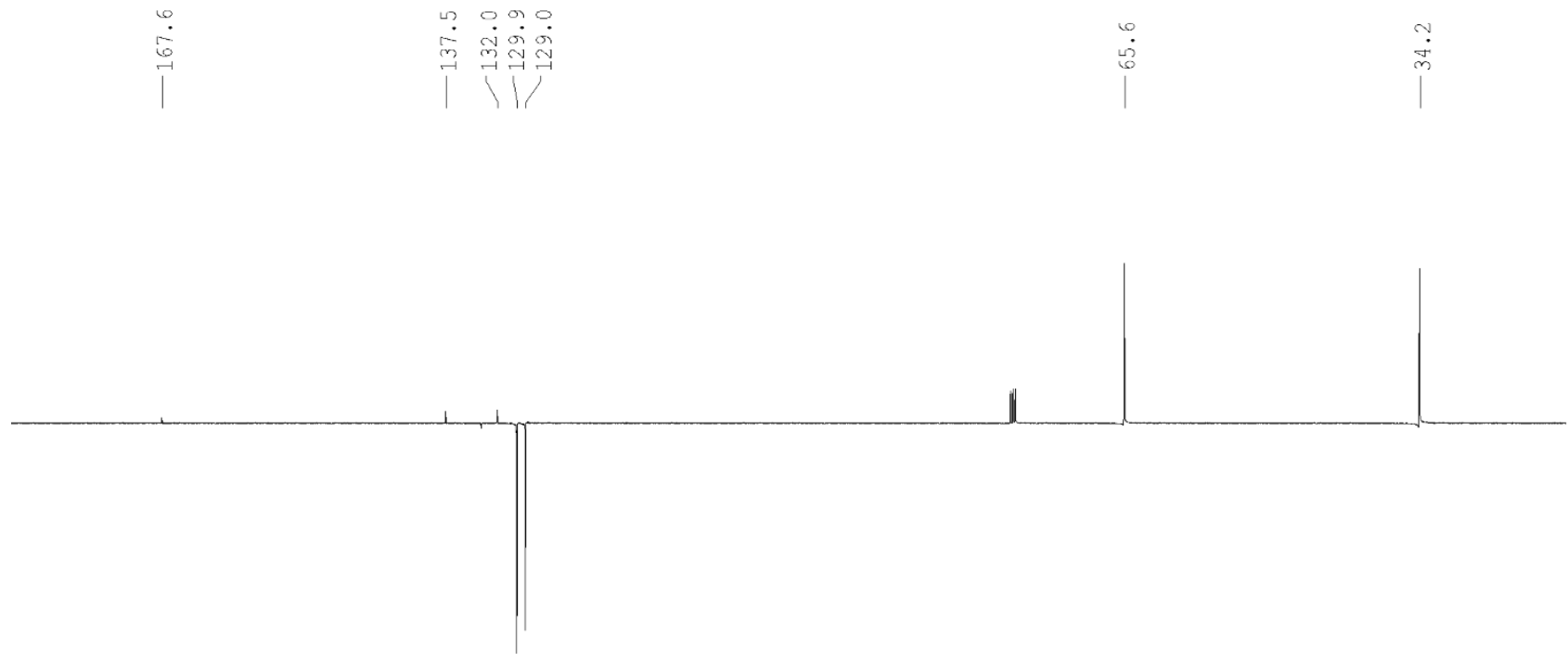

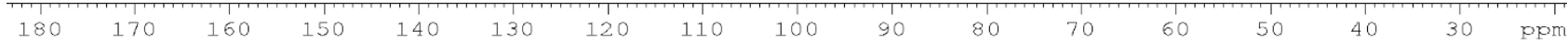


${ }^{1} \mathrm{H}$ NMR (400 MHz, $\mathrm{CDCl}_{3}, 298 \mathrm{~K}$, TMS) of 2-(4-bromophenyl)-4,5-dihydrothiazole, 4d

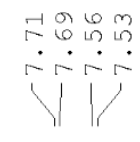

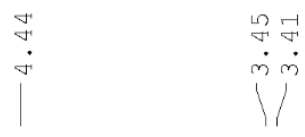<smiles>Brc1ccc(C2=NCCS2)cc1</smiles>

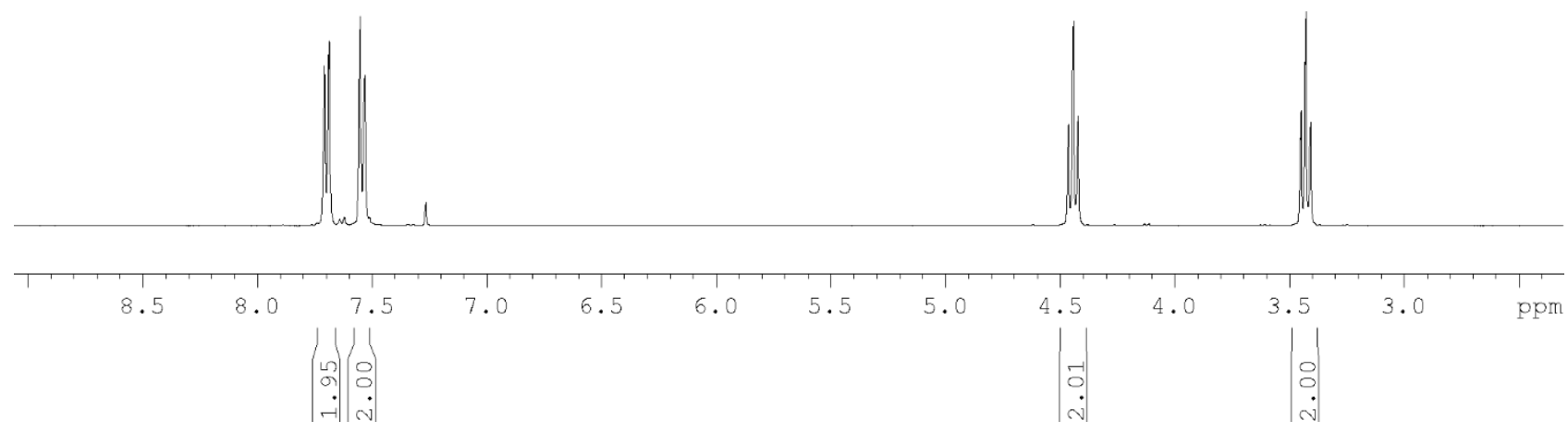

${ }^{13} \mathrm{C}-\left\{{ }^{1} \mathrm{H}\right\}$ NMR (75 MHz, $\left.\mathrm{CDCl} 3,298 \mathrm{~K}\right)$ of 2-(4-bromophenyl)-4,5-dihydrothiazole, $4 \mathbf{d}$
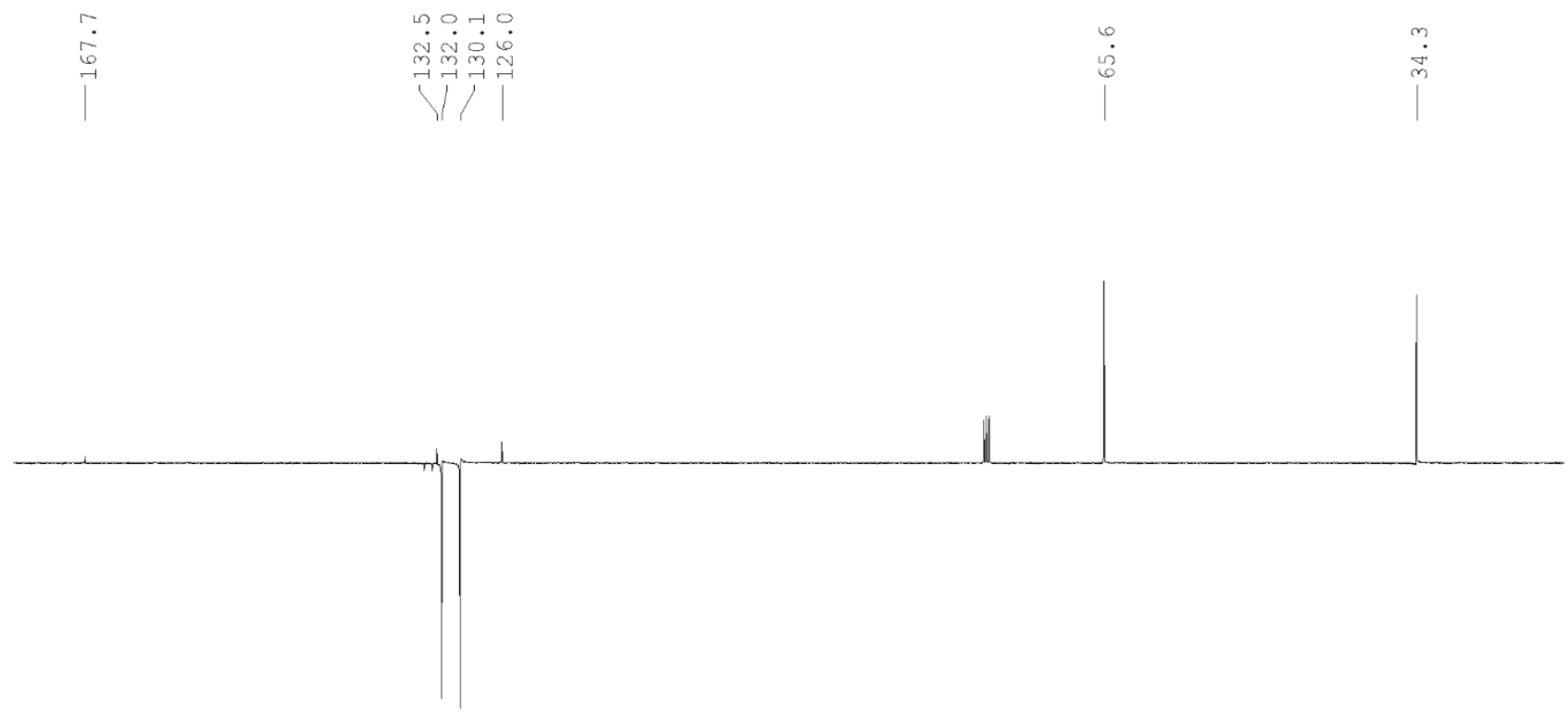

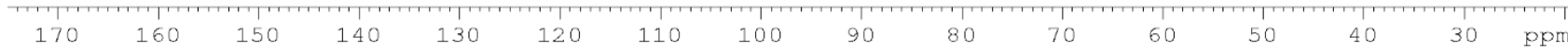


${ }^{1} \mathrm{H}$ NMR (400 MHz, $\mathrm{CDCl}_{3}, 298 \mathrm{~K}$, TMS) of 2-p-tolyl-4,5-dihydrothiazole, $4 \mathbf{e}$ 帮

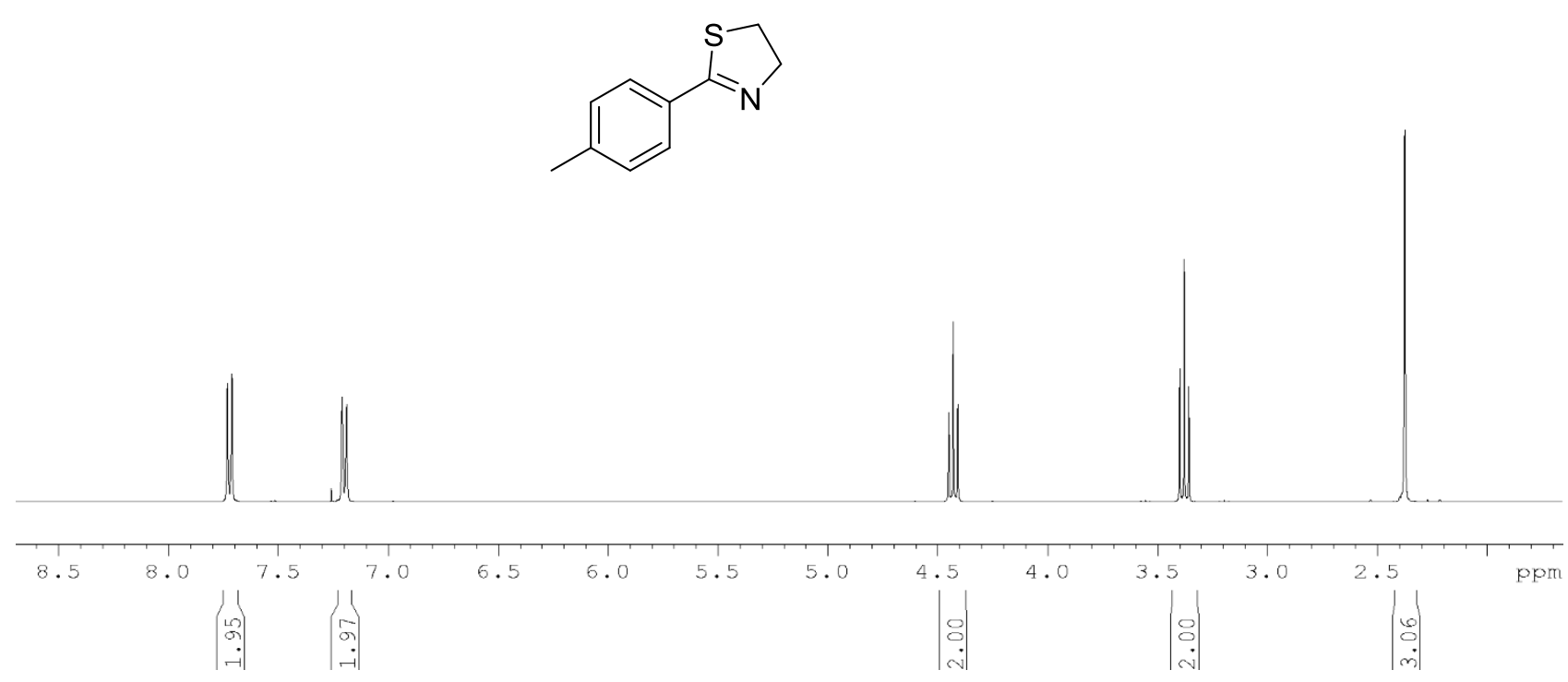

${ }^{13} \mathrm{C}-\left\{{ }^{1} \mathrm{H}\right\}$ NMR (75 MHz, CDCl $\left.3,298 \mathrm{~K}\right)$ of 2-p-tolyl-4,5-dihydrothiazole, $4 \mathbf{e}$

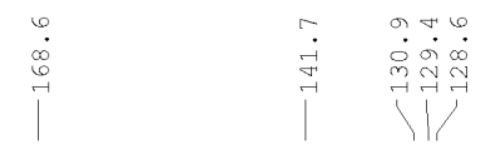
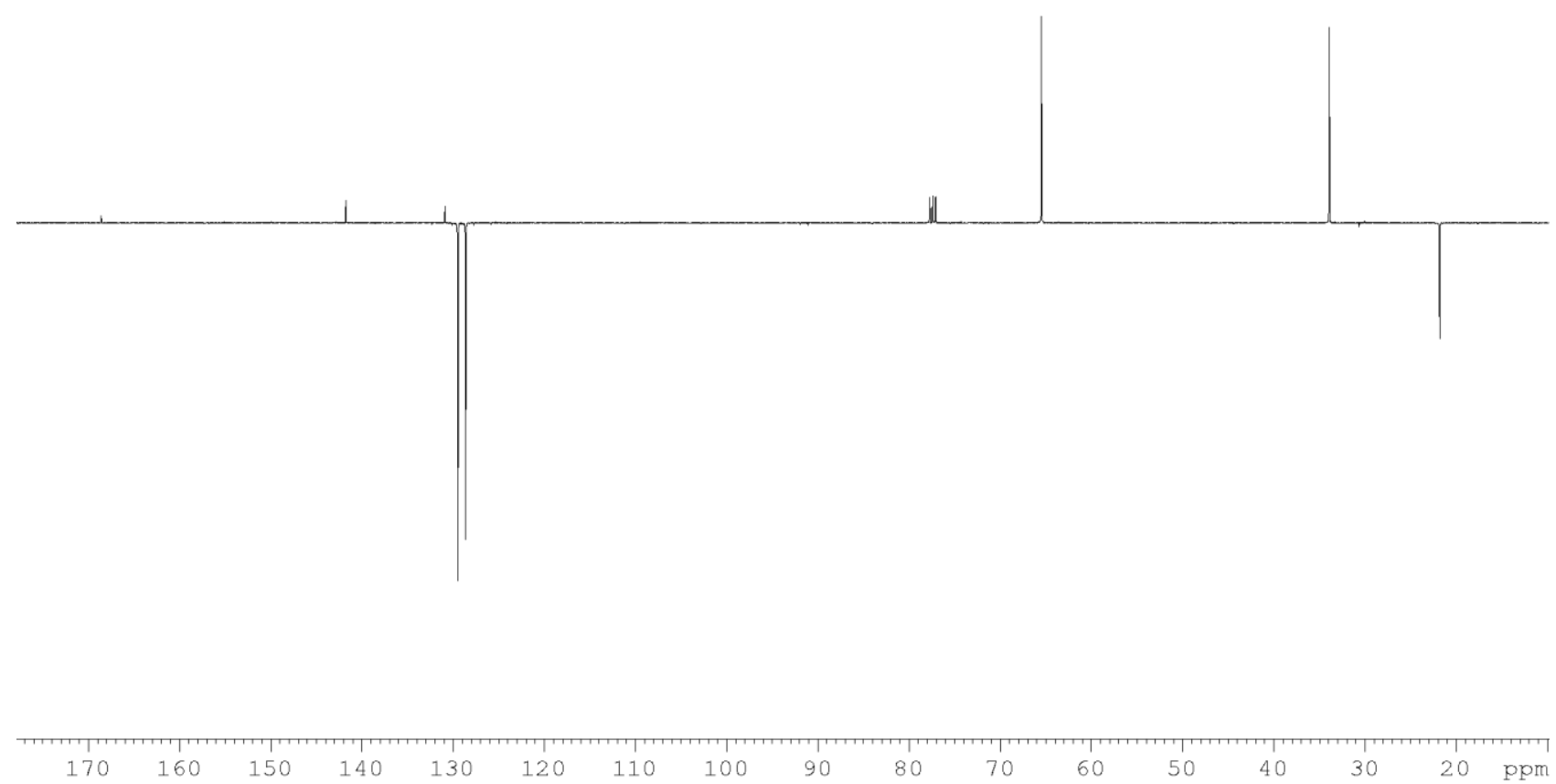
${ }^{1} \mathrm{H}$ NMR (500 MHz, $\mathrm{CDCl}_{3}, 298 \mathrm{~K}$, TMS) of 2-(4-methoxyphenyl)-4,5-dihydrothiazole, $4 \mathbf{f}$

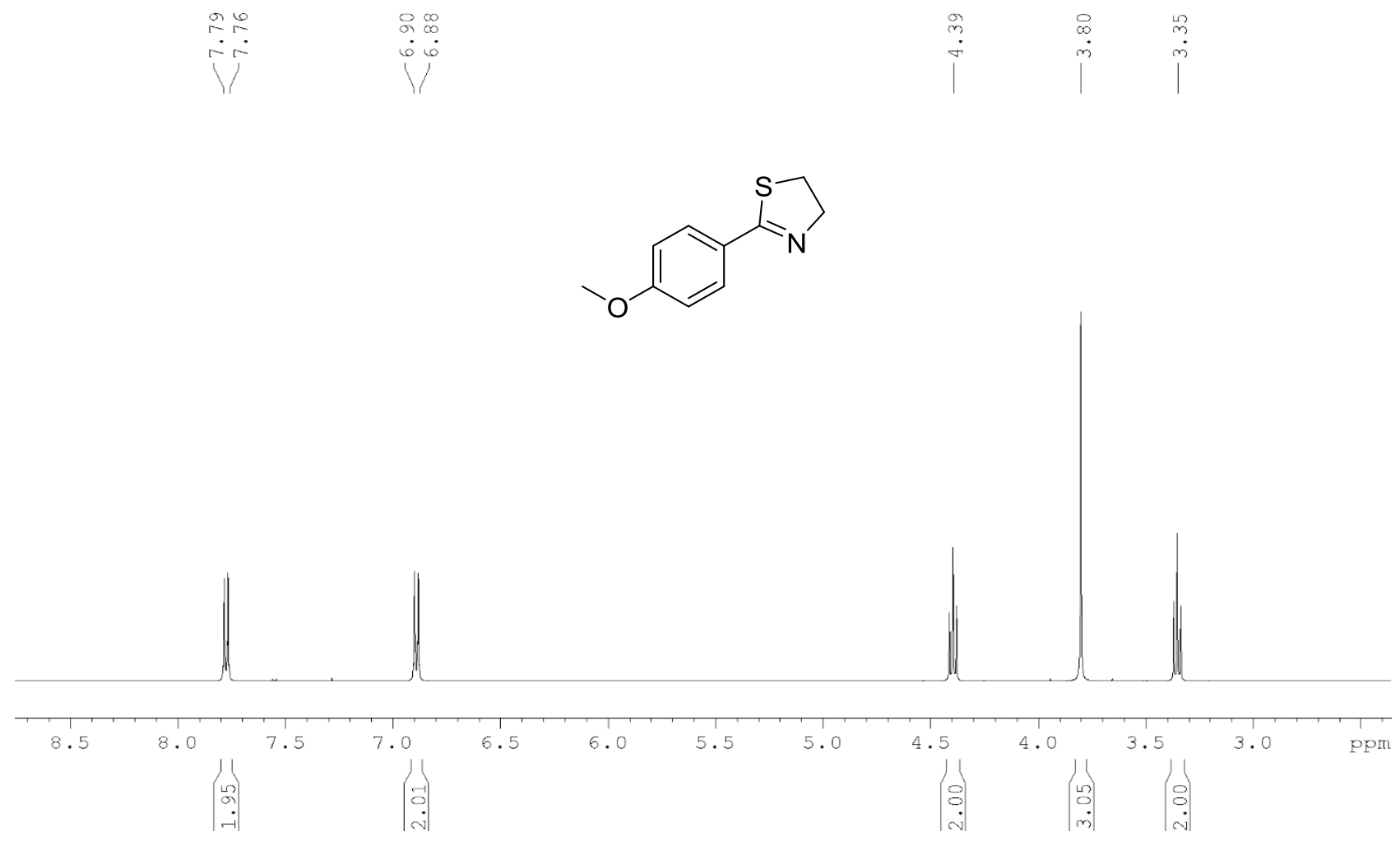

${ }^{13} \mathrm{C}-\left\{{ }^{1} \mathrm{H}\right\}$ NMR $\left(125.7 \mathrm{MHz}, \mathrm{CDCl}_{3}, 298 \mathrm{~K}\right)$ of 2-(4-methoxyphenyl)-4,5-dihydrothiazole, $\mathbf{4 f}$
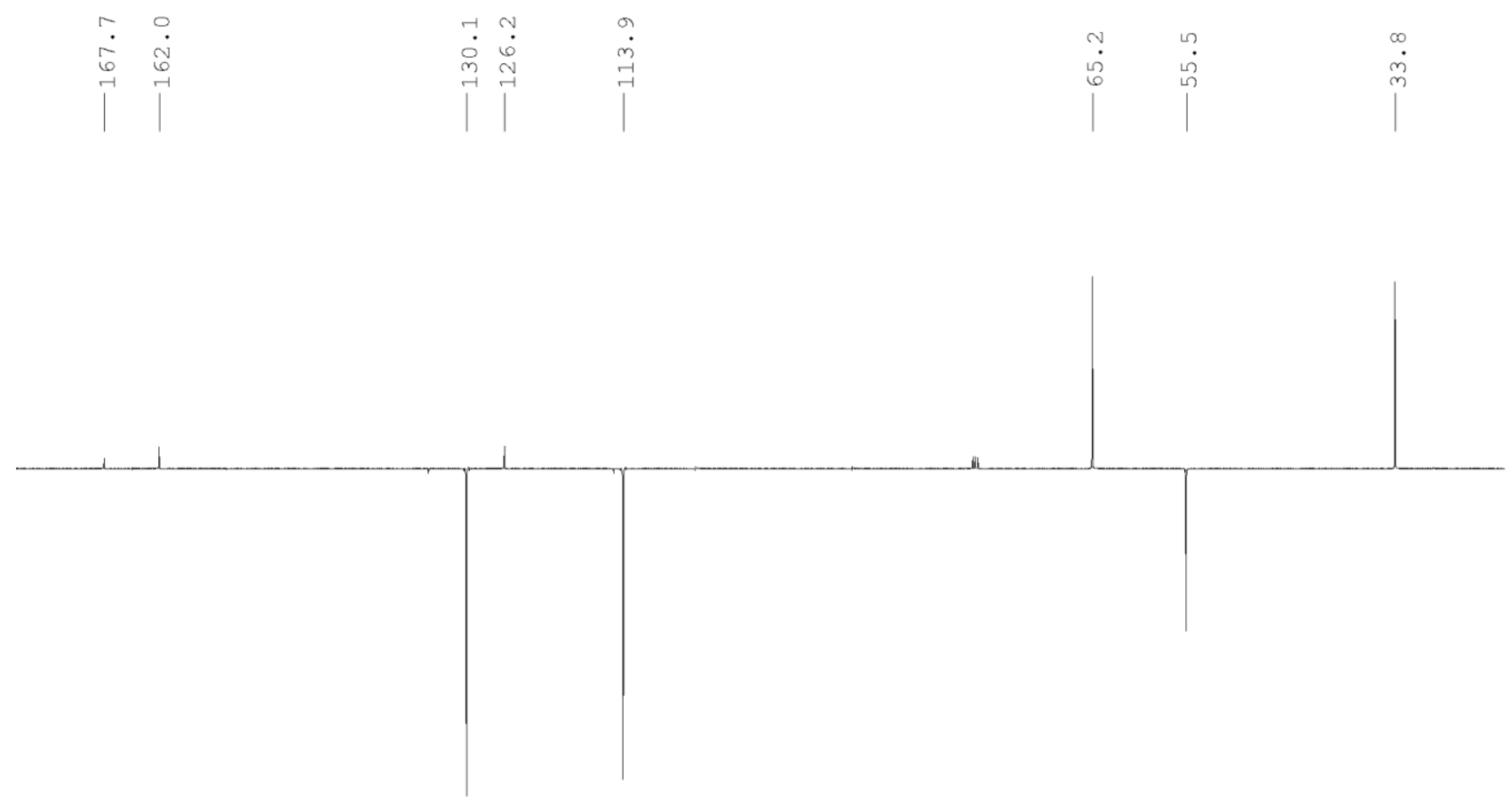
${ }^{1} \mathrm{H}$ NMR (400 MHz, $\mathrm{CDCl}_{3}, 298 \mathrm{~K}$, TMS) of 4-(4,5-dihydrothiazol-2-yl)aniline, $\mathbf{4 g}$

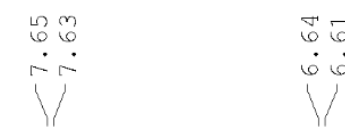<smiles>Nc1ccc(C2=NCCS2)cc1</smiles>

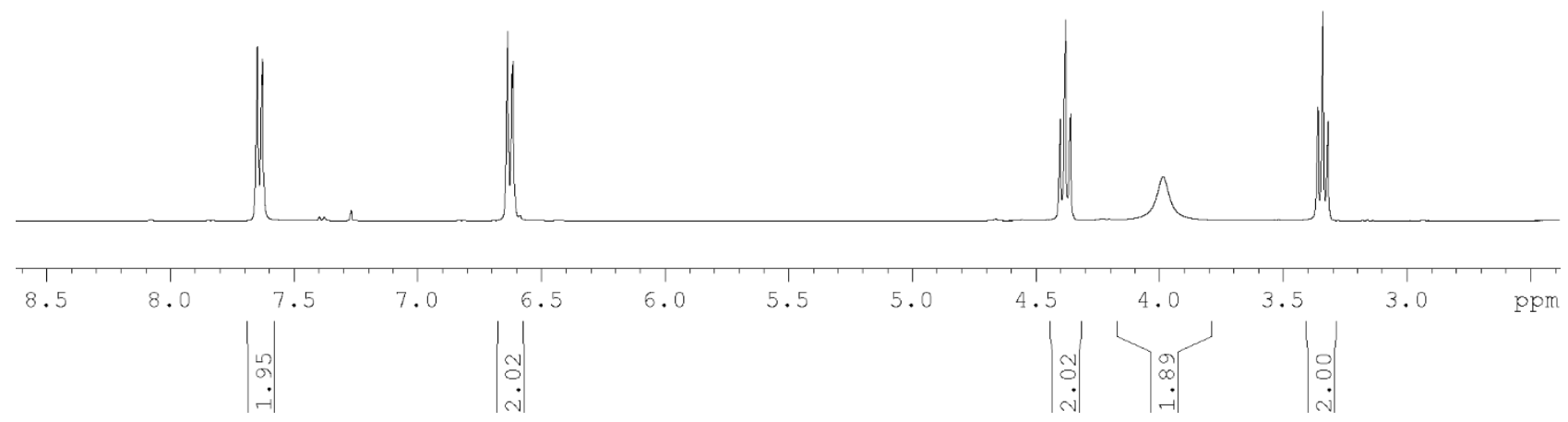

${ }^{13} \mathrm{C}-\left\{{ }^{1} \mathrm{H}\right\}$ NMR (75 MHz, $\left.\mathrm{CDCl}_{3}, 298 \mathrm{~K}\right)$ of 4-(4,5-dihydrothiazol-2-yl)aniline, $\mathbf{4 g}$
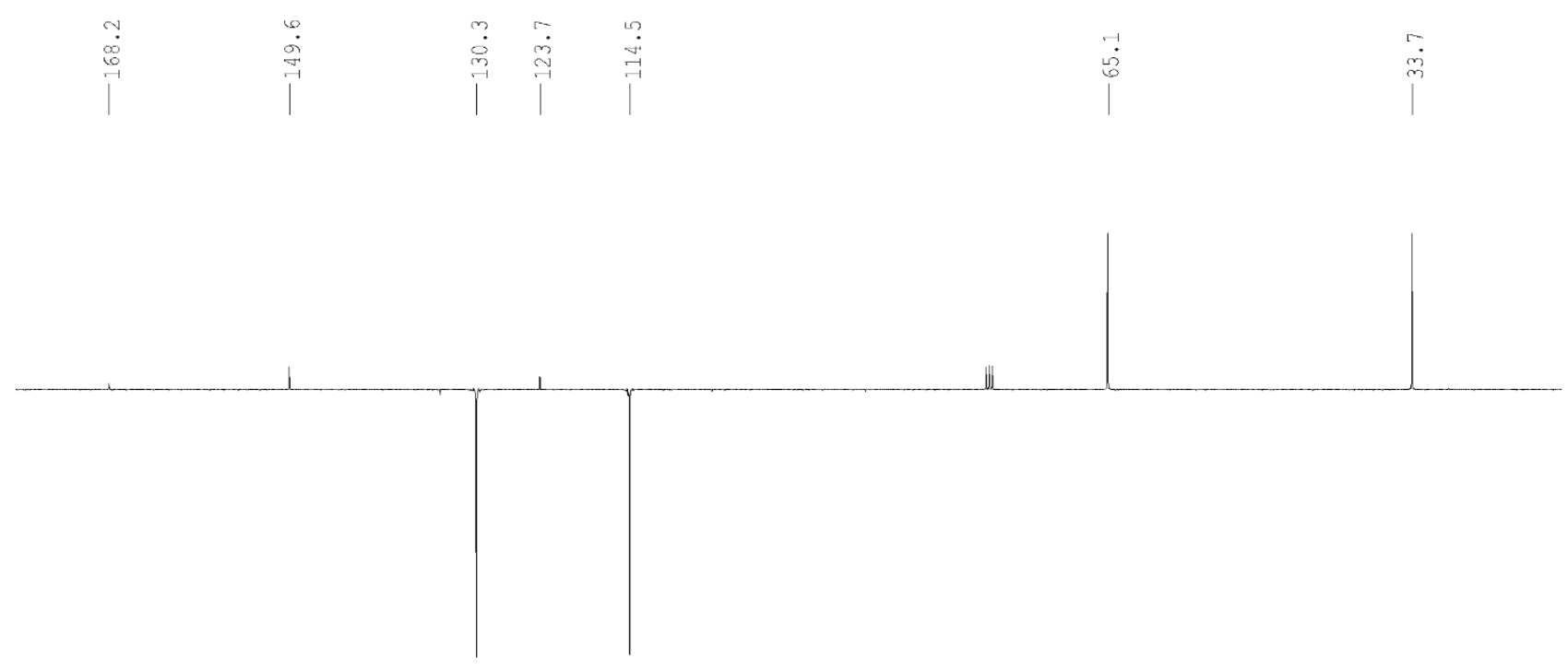

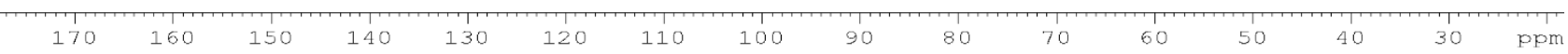


${ }^{1} \mathrm{H}$ NMR (400 MHz, $\mathrm{CDCl}_{3}, 298 \mathrm{~K}$, TMS) of 2-(3-bromophenyl)-4,5-dihydrothiazole, $\mathbf{4 h}$
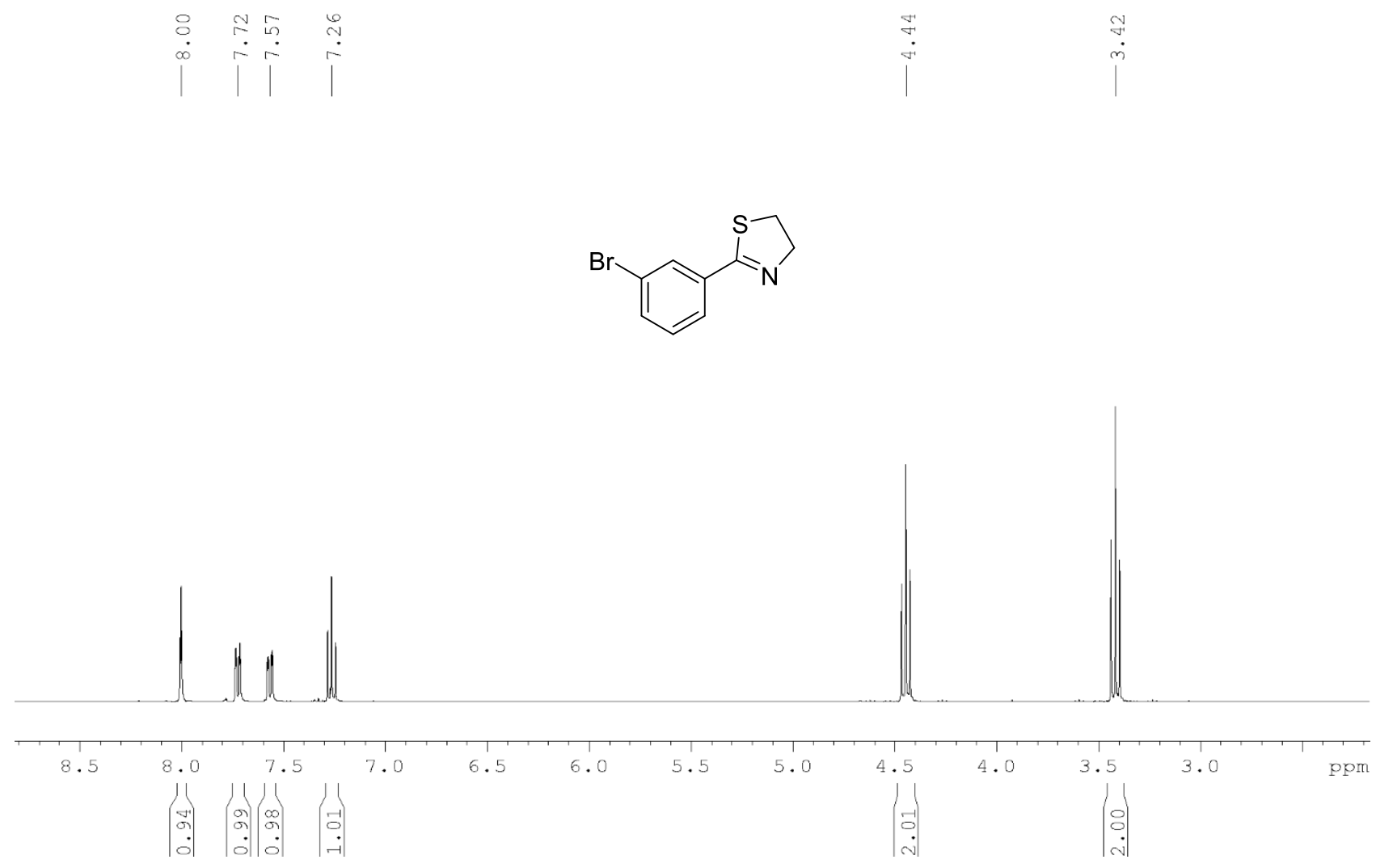

${ }^{13} \mathrm{C}-\left\{{ }^{1} \mathrm{H}\right\}$ NMR (75 MHz, $\left.\mathrm{CDCl}_{3}, 298 \mathrm{~K}\right)$ of 2-(3-bromophenyl)-4,5-dihydrothiazole, $4 \mathbf{h}$
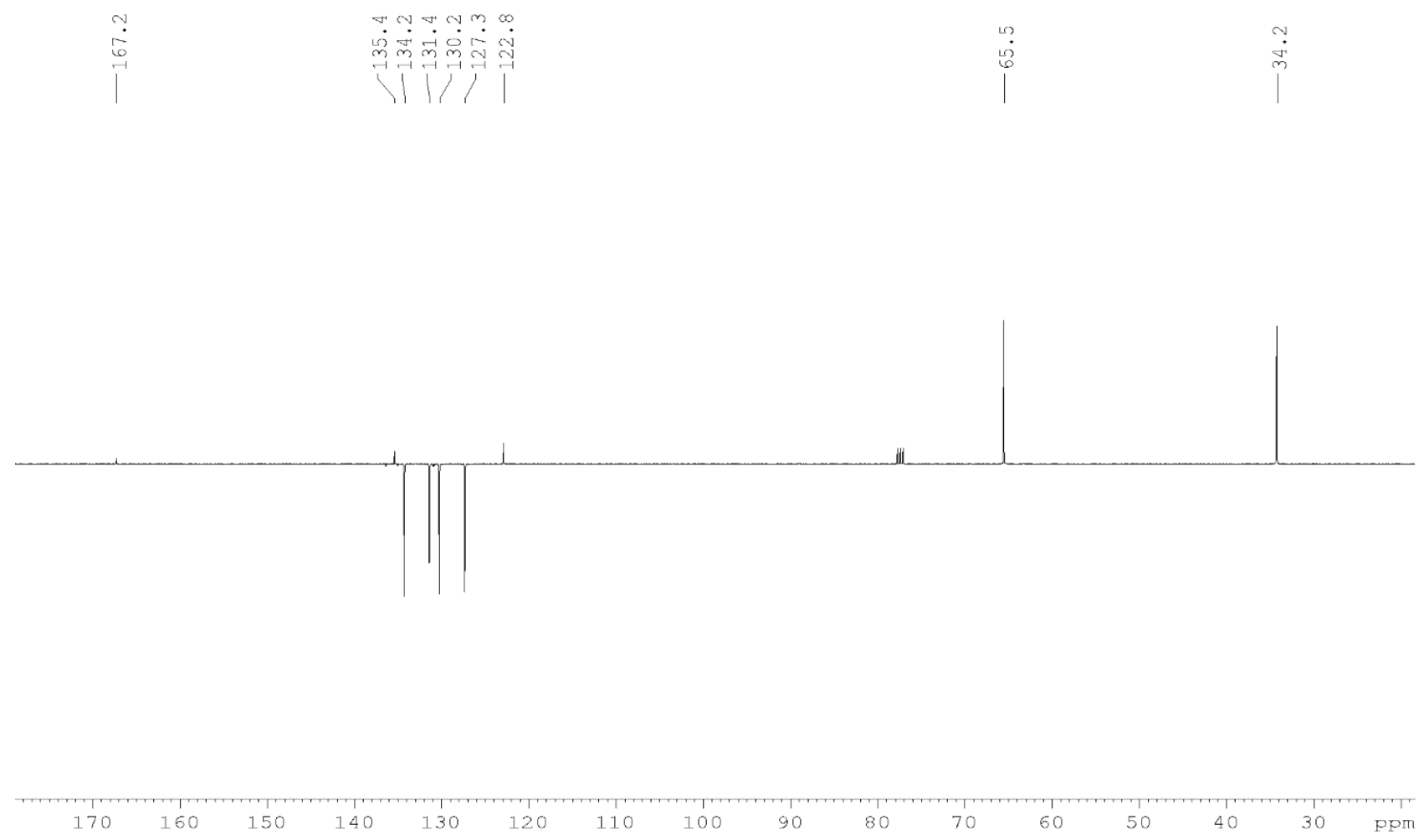
${ }^{1} \mathrm{H}$ NMR (500 MHz, $\mathrm{CDCl}_{3}, 298 \mathrm{~K}$, TMS) of 2-m-tolyl-4,5-dihydrothiazole, $4 \mathbf{i}$

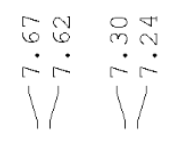<smiles>Cc1cccc(C2=NCCS2)c1</smiles>

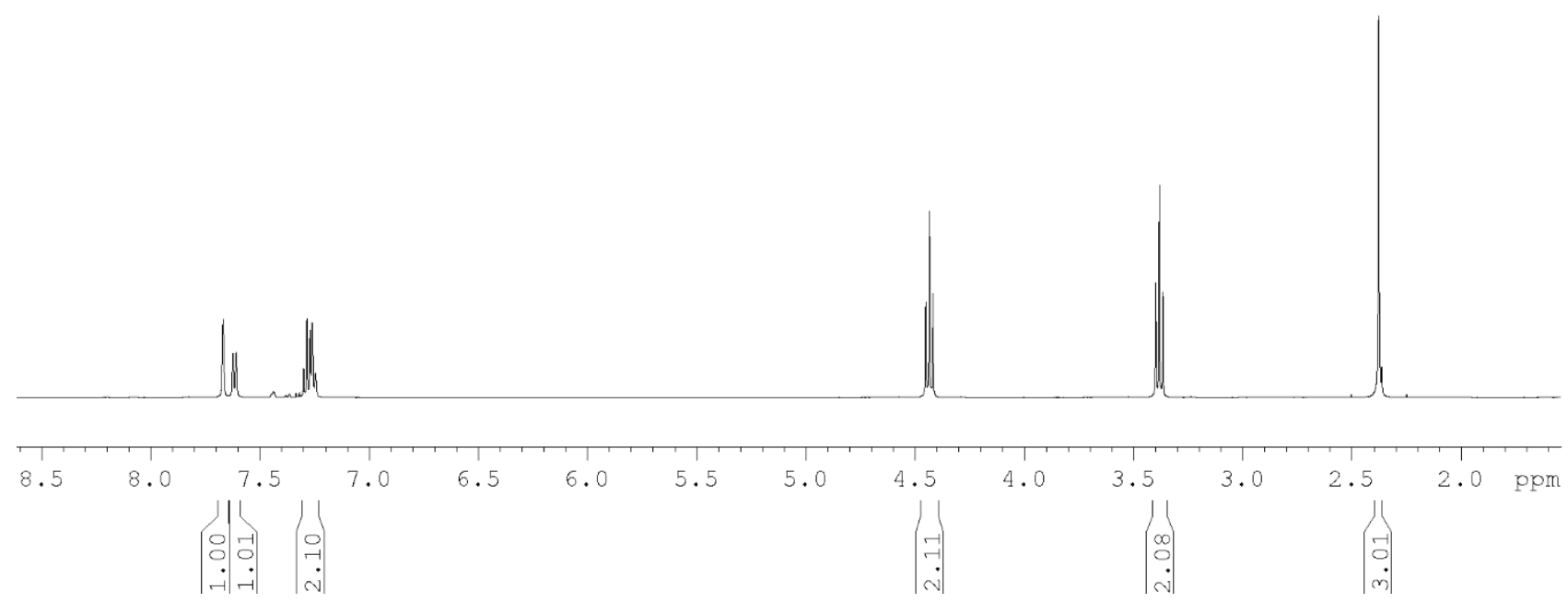

${ }^{13} \mathrm{C}-\left\{{ }^{1} \mathrm{H}\right\}$ NMR (125.7 MHz, CDCl $\left.3,298 \mathrm{~K}\right)$ of 2-m-tolyl-4,5-dihydrothiazole, $4 \mathbf{i}$
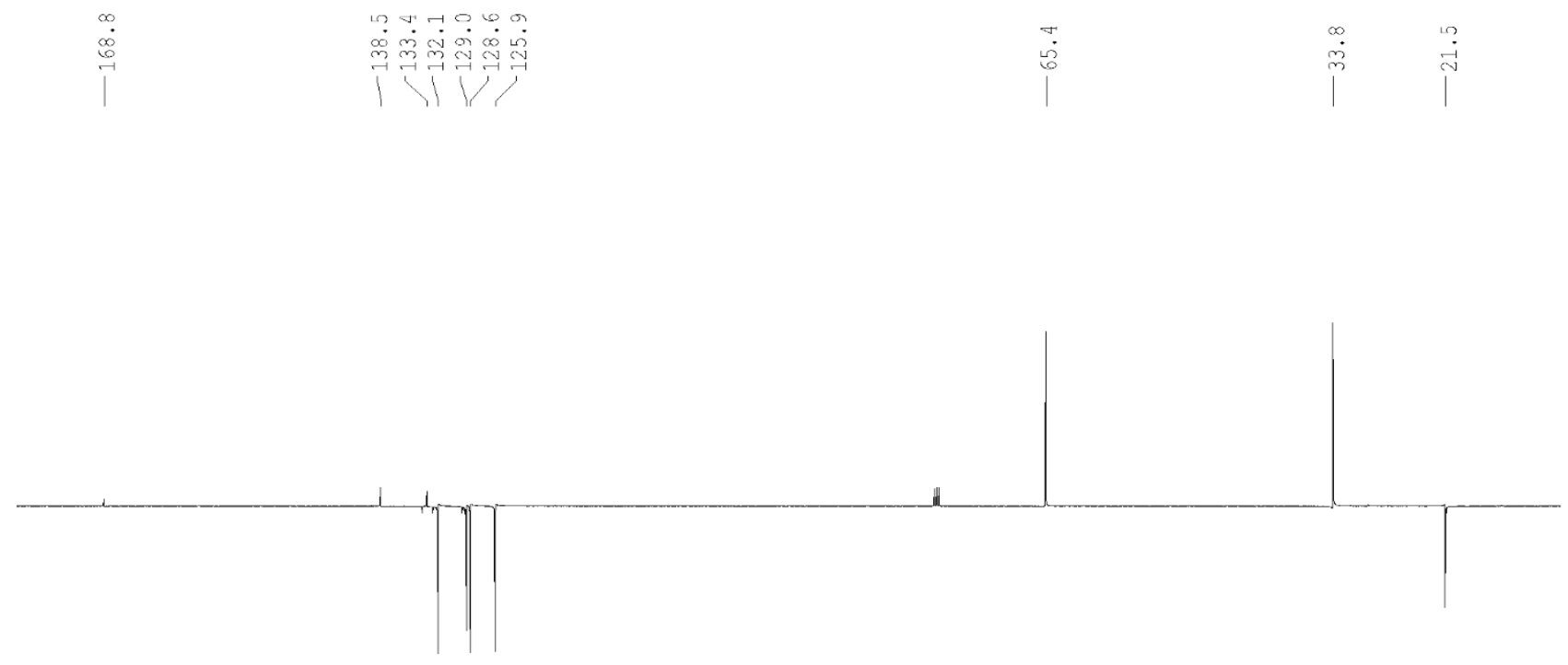
${ }^{1} \mathrm{H}$ NMR (400 MHz, $\mathrm{CDCl}_{3}, 298 \mathrm{~K}$, TMS) of 3-(4,5-dihydrothiazol-2-yl)aniline, $\mathbf{4 j}$

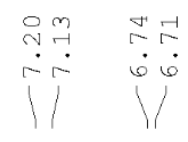

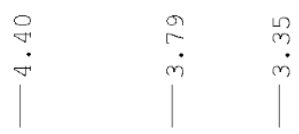<smiles>Nc1cccc(C2=NCCS2)c1</smiles>

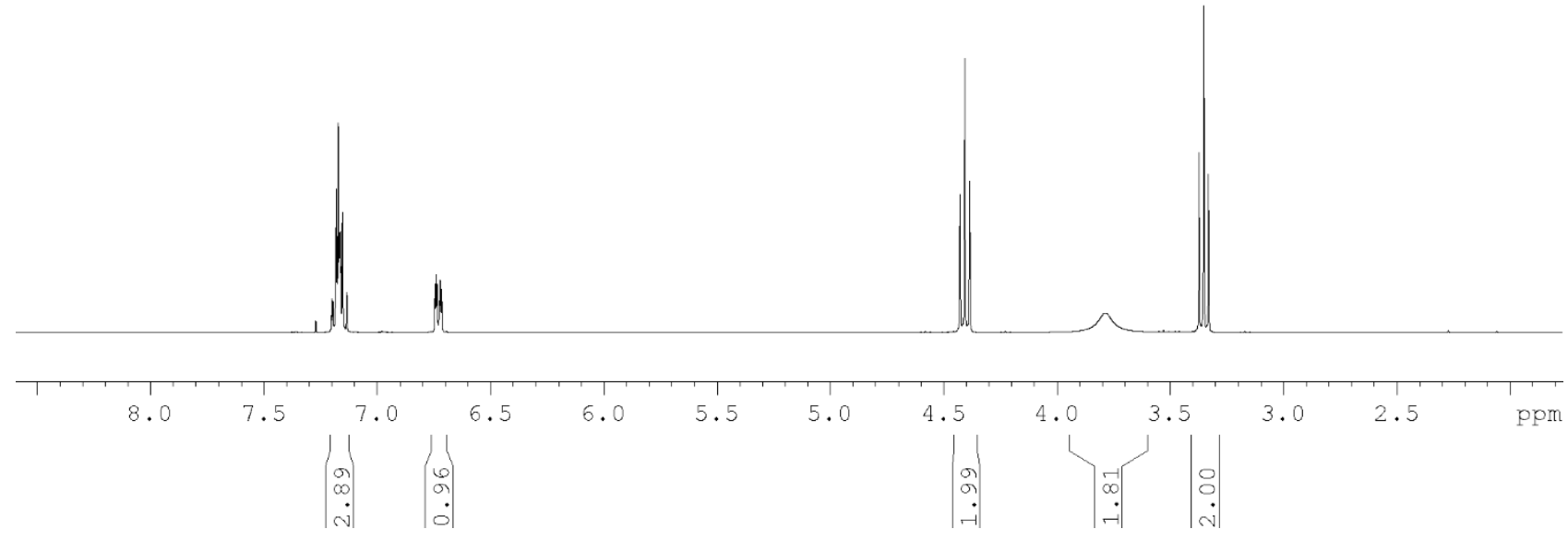

${ }^{13} \mathrm{C}-\left\{{ }^{1} \mathrm{H}\right\}$ NMR (75 MHz, $\left.\mathrm{CDCl}_{3}, 298 \mathrm{~K}\right)$ of 3-(4,5-dihydrothiazol-2-yl)aniline, $4 \mathbf{j}$
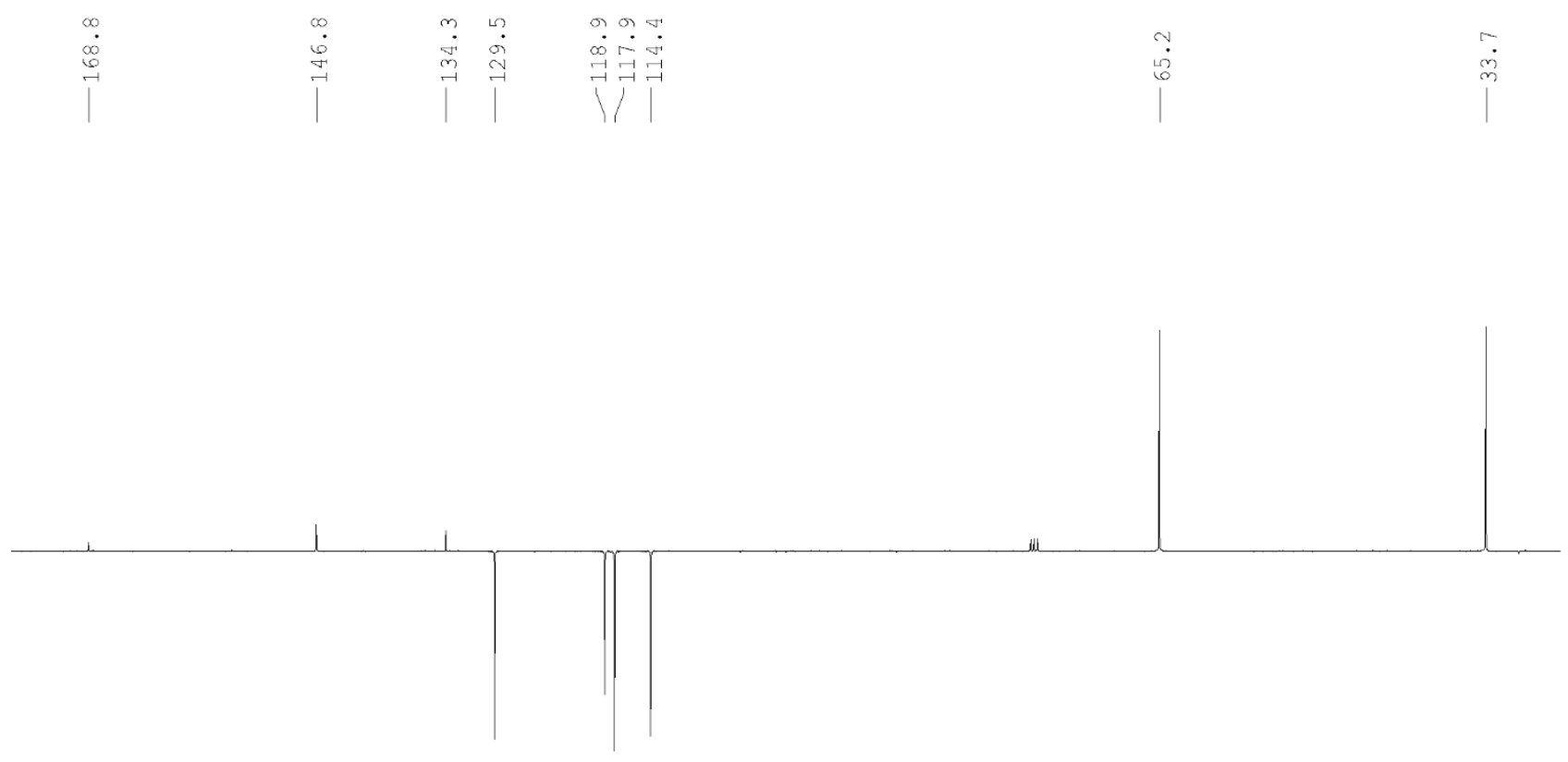
${ }^{1} \mathrm{H}$ NMR (400 MHz, $\left.\mathrm{CDCl}_{3}, 298 \mathrm{~K}, \mathrm{TMS}\right)$ of 2-(3,5-difluorophenyl)-4,5-dihydrothiazole, 4k

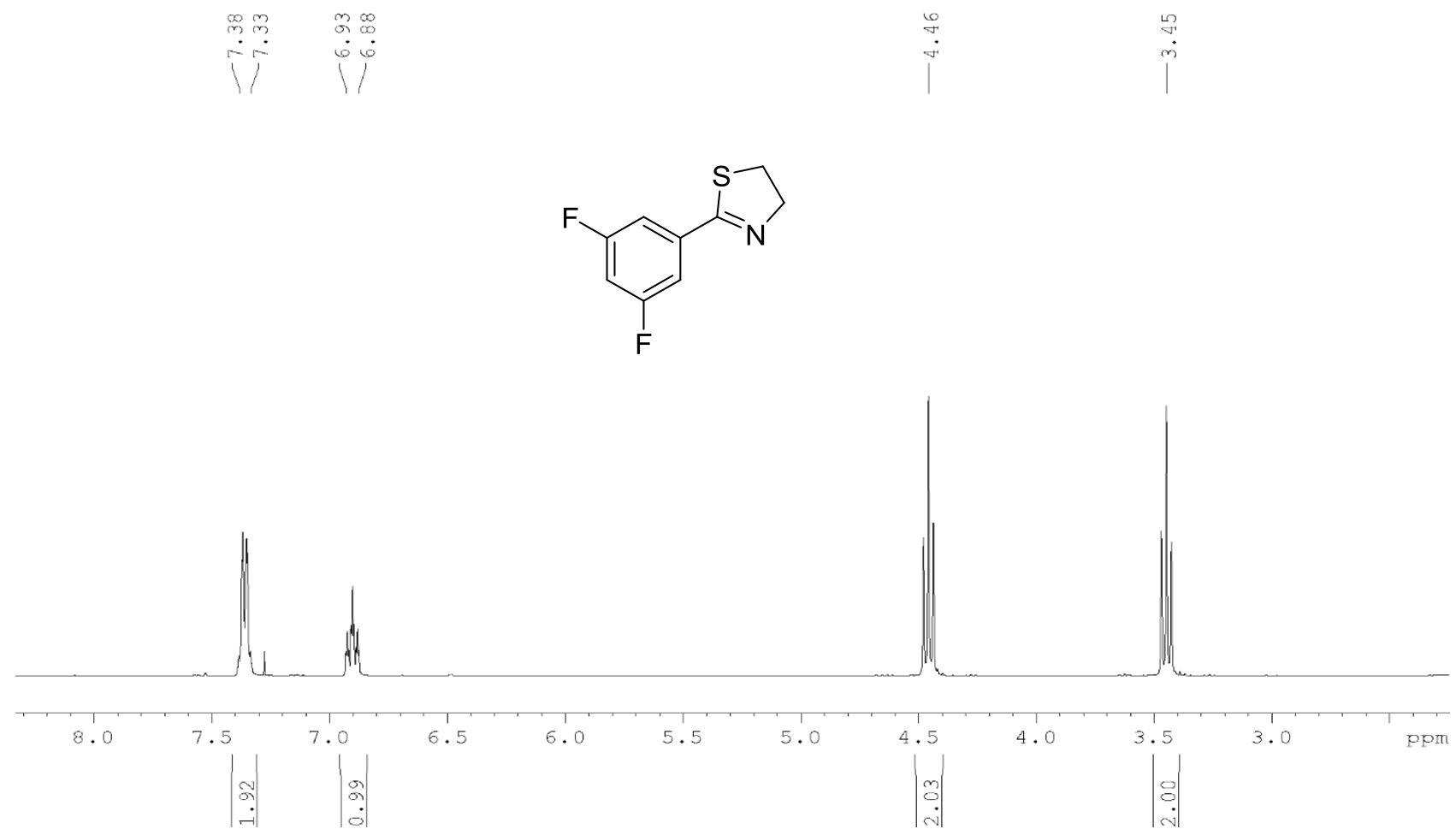

${ }^{13} \mathrm{C}-\left\{{ }^{1} \mathrm{H}\right\}$ NMR $\left(75 \mathrm{MHz}, \mathrm{CDCl}_{3}, 298 \mathrm{~K}\right)$ of 2-(3,5-difluorophenyl)-4,5-dihydrothiazole, $4 \mathbf{k}$
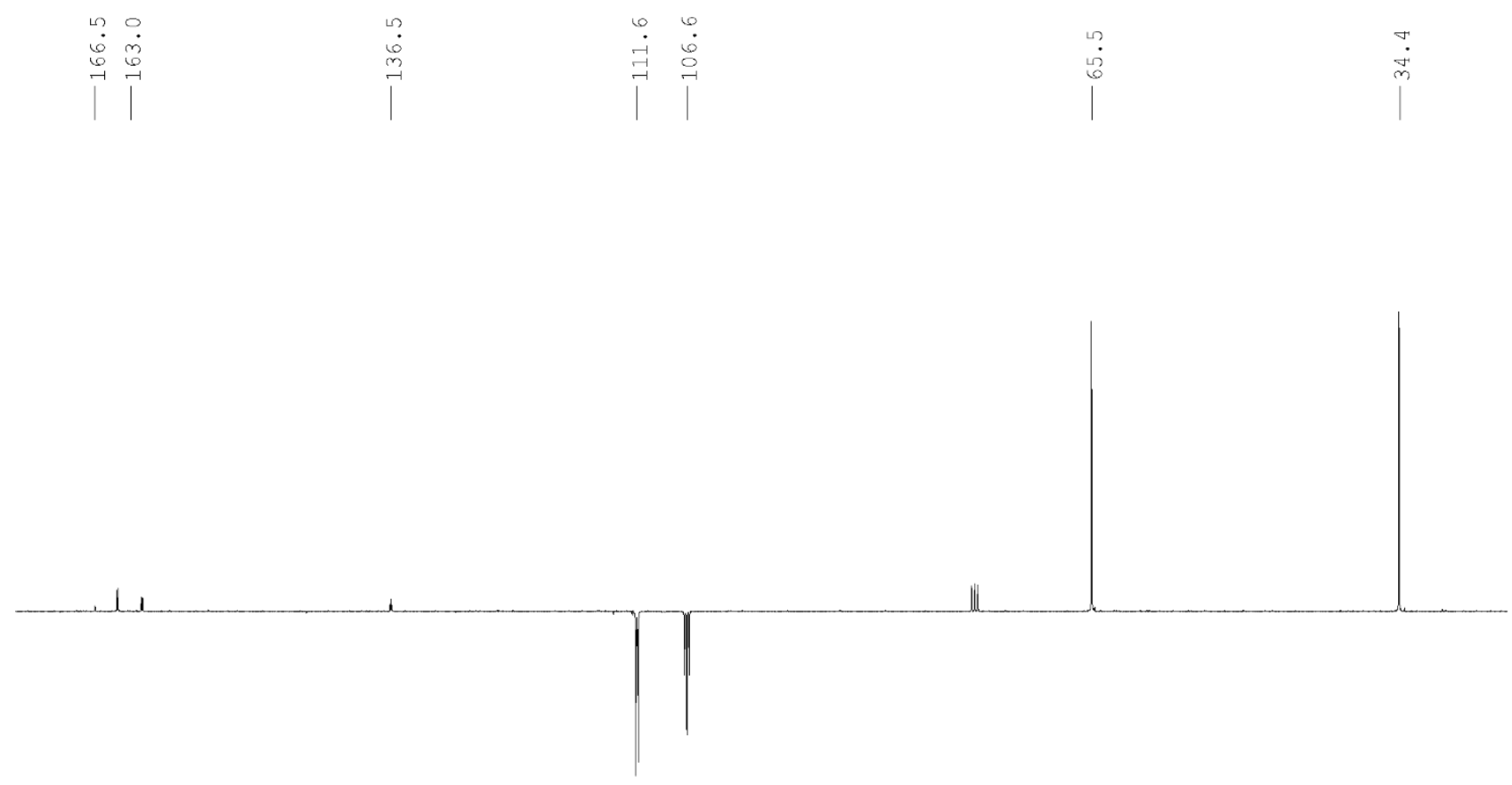

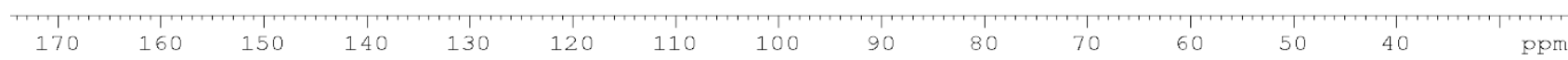


${ }^{19} \mathrm{~F}-\left\{{ }^{1} \mathrm{H}\right\}$ NMR (376 MHz, 298K) of 2-(3,5-difluorophenyl)-4,5-dihydrothiazole, $4 \mathbf{k}$

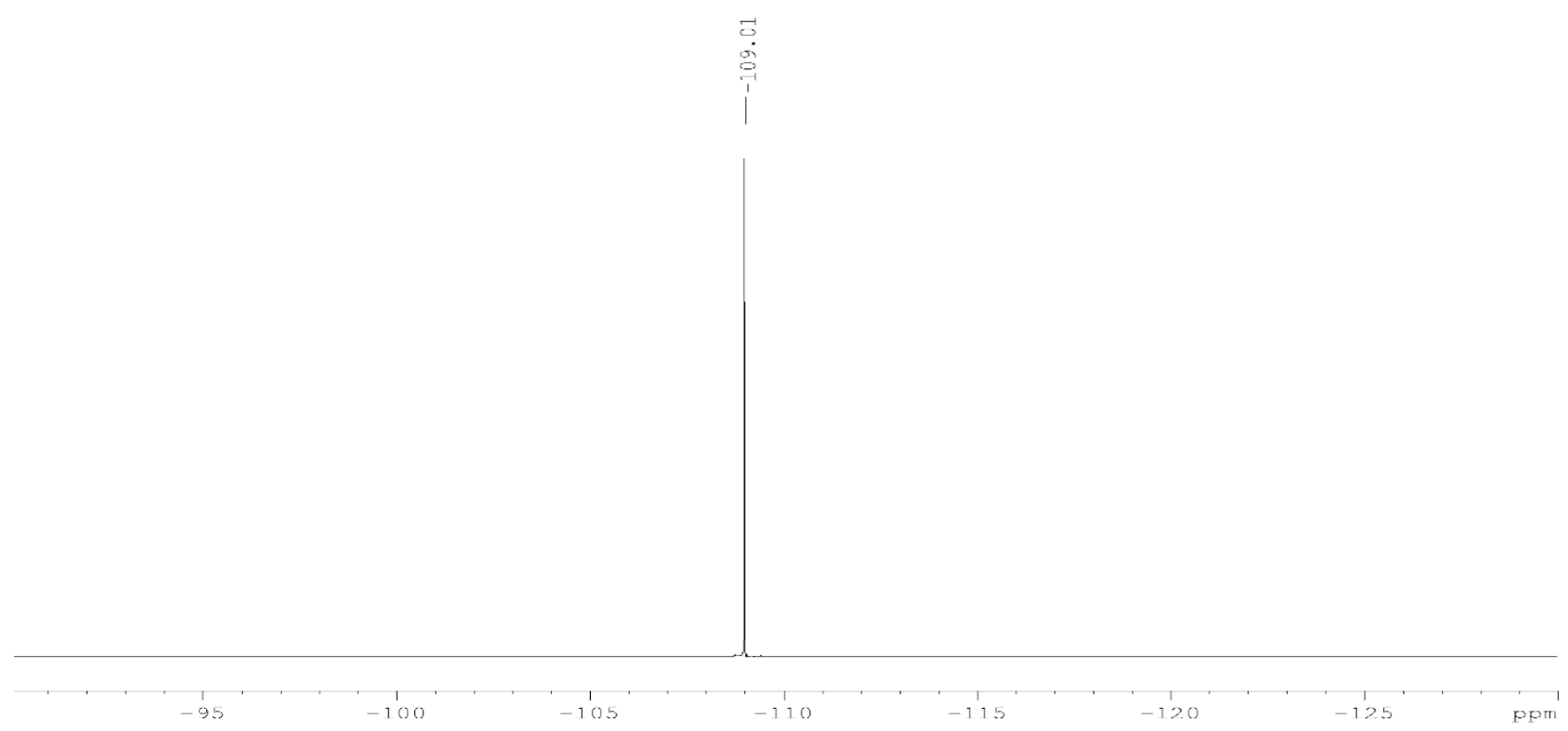


${ }^{1} \mathrm{H}$ NMR (500 MHz, $\left.\mathrm{CDCl}_{3}, 298 \mathrm{~K}, \mathrm{TMS}\right)$ of 2-(2-chlorophenyl)-4,5-dihydrothiazole, $4 \mathbf{l}$

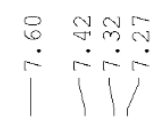<smiles>Clc1ccccc1C1=NCCS1</smiles>
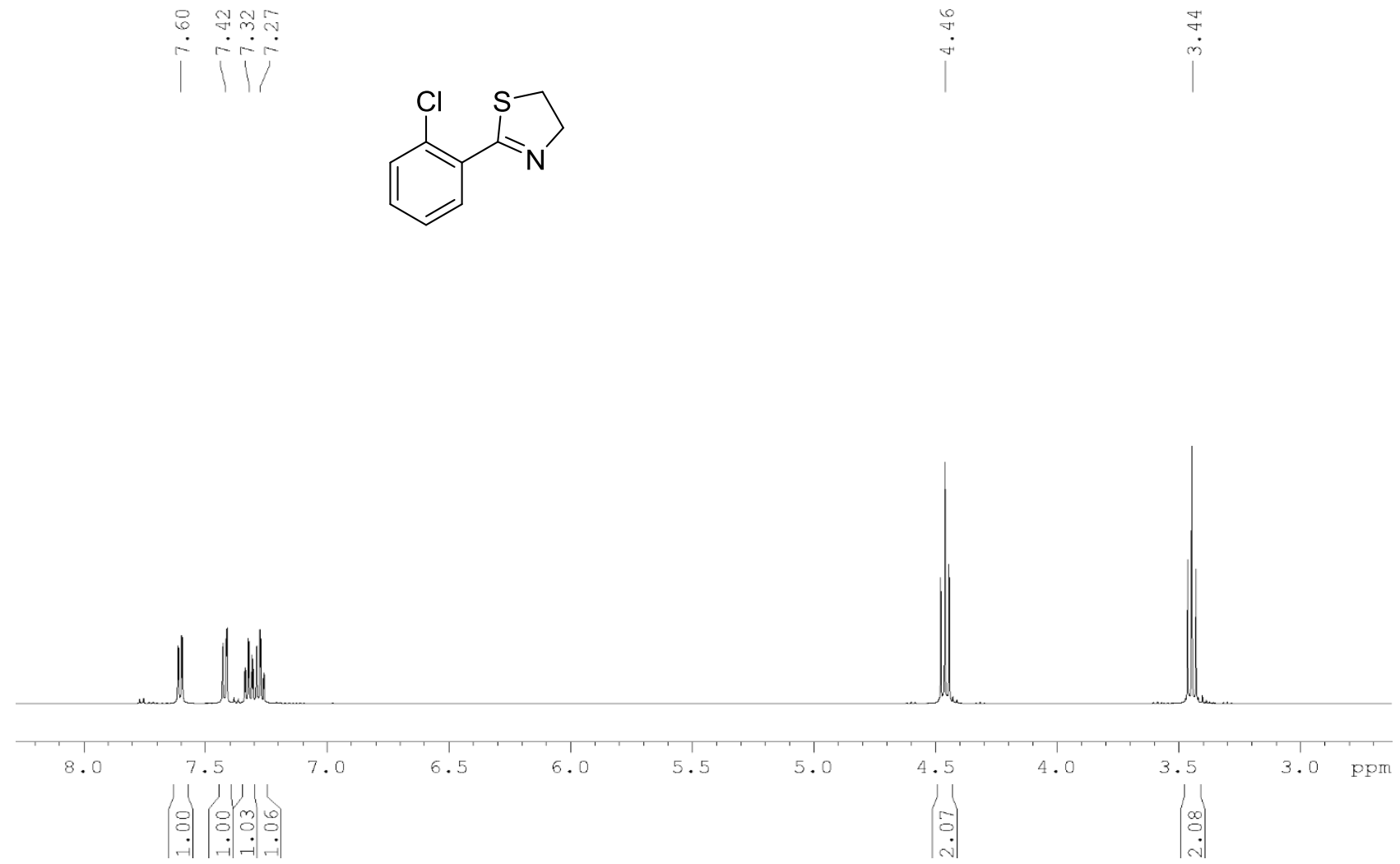

${ }^{13} \mathrm{C}-\left\{{ }^{1} \mathrm{H}\right\}$ NMR $\left(125.7 \mathrm{MHz}, \mathrm{CDCl}_{3}, 298 \mathrm{~K}\right)$ of 2-(2-chlorophenyl)-4,5-dihydrothiazole, $4 \mathbf{l}$
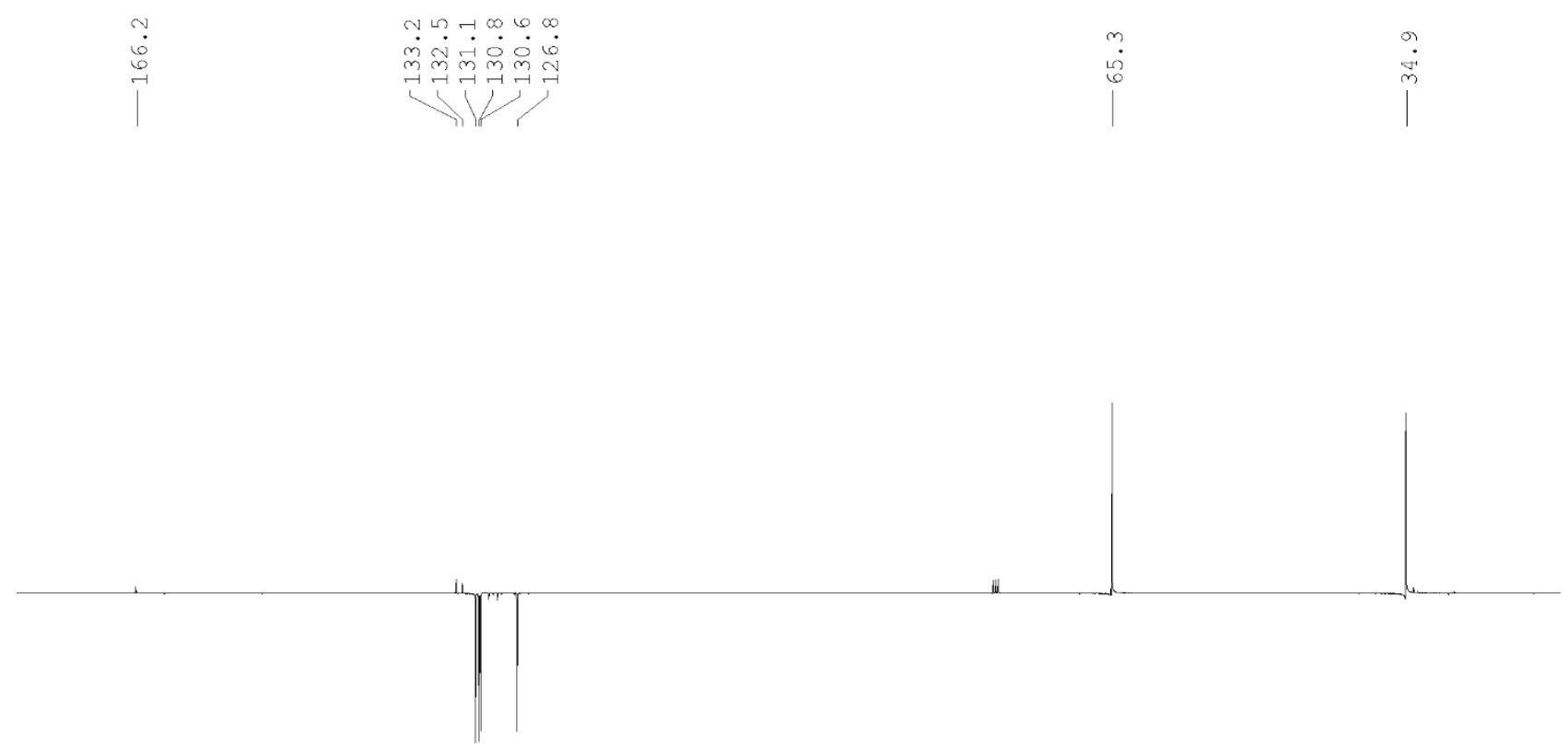

$\begin{array}{llllllllllllllll}170 & 160 & 150 & 140 & 130 & 120 & 110 & 100 & 90 & 80 & 70 & 60 & 50 & 40 & 30 & \text { ppm }\end{array}$


${ }^{1} \mathrm{H}$ NMR (400 MHz, $\mathrm{CDCl}_{3}, 298 \mathrm{~K}$, TMS) of 2-(2-bromophenyl)-4,5-dihydrothiazole, $\mathbf{4 m}$

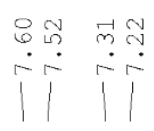<smiles>Brc1ccccc1C1=NCCS1</smiles>
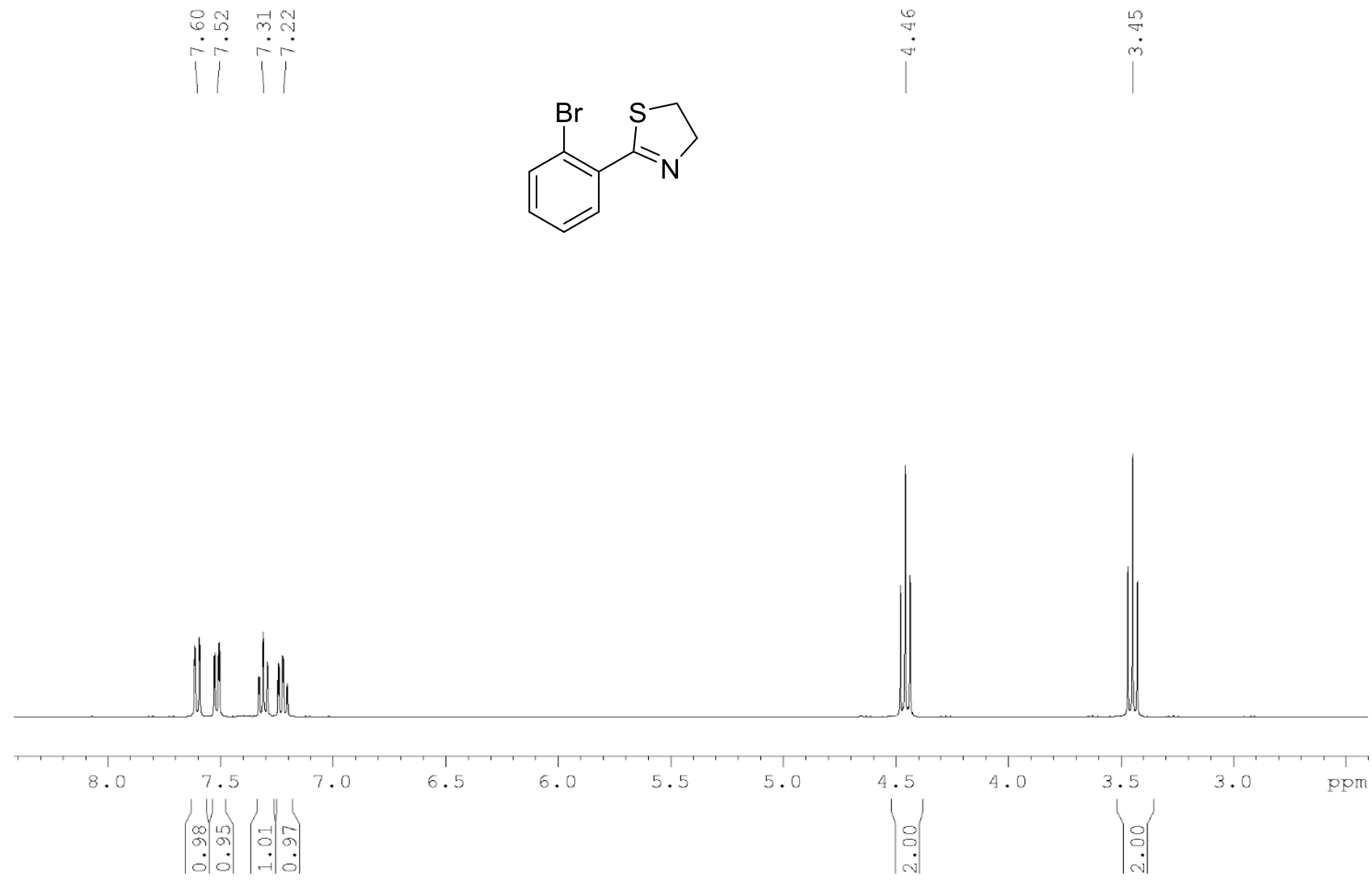

${ }^{13} \mathrm{C}-\left\{{ }^{1} \mathrm{H}\right\}$ NMR (75 MHz, CDCl $\left.3,298 \mathrm{~K}\right)$ of 2-(2-bromophenyl)-4,5-dihydrothiazole, $4 \mathbf{m}$
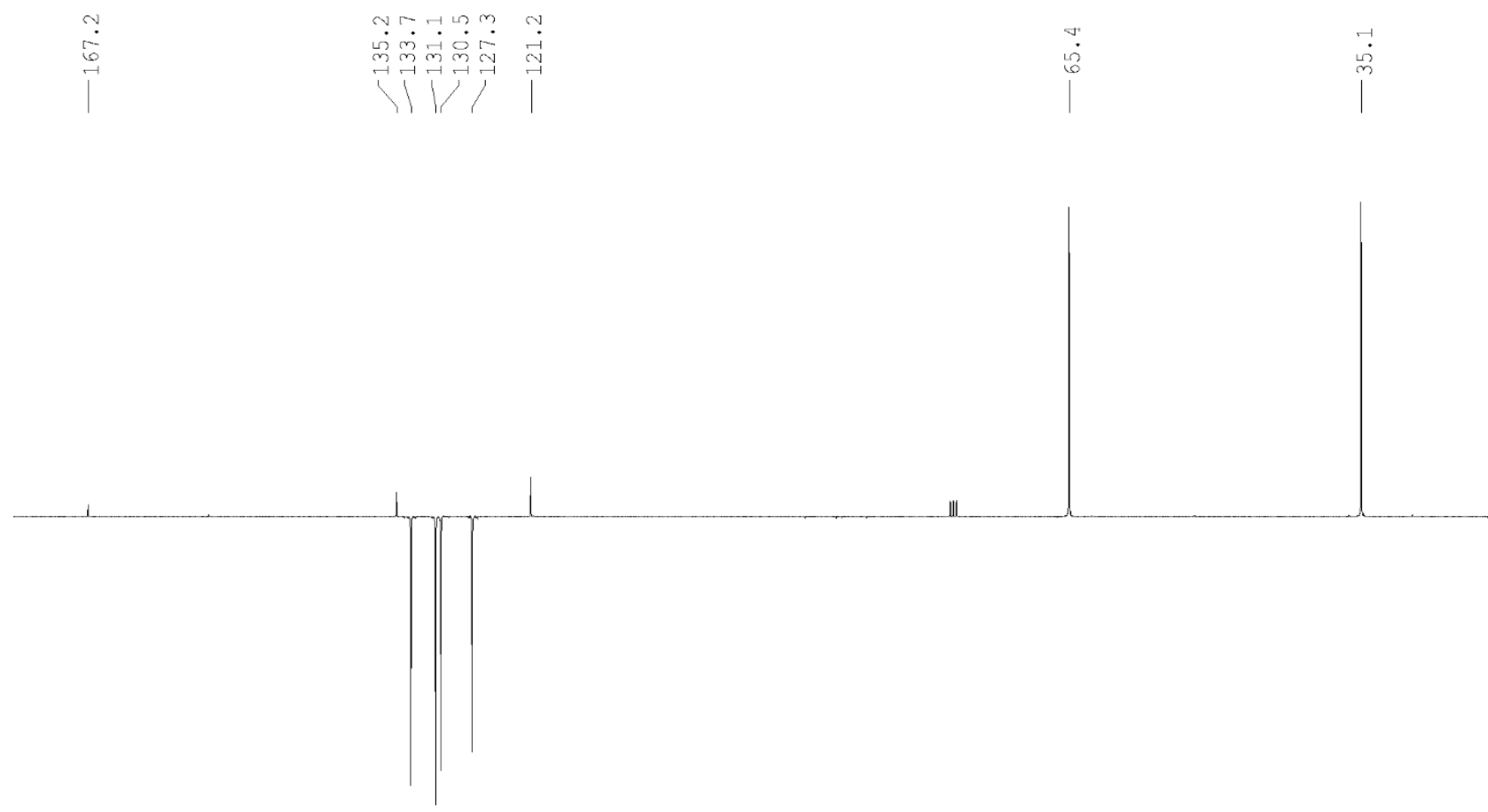

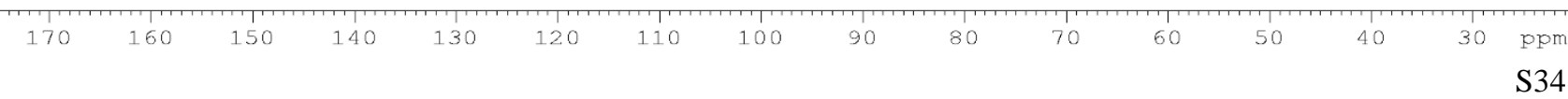


${ }^{1} \mathrm{H}$ NMR (400 MHz, CDCl, $298 \mathrm{~K}$, TMS) of 2-(4,5-dihydrothiazol-2-yl)aniline, $4 \mathbf{n}$
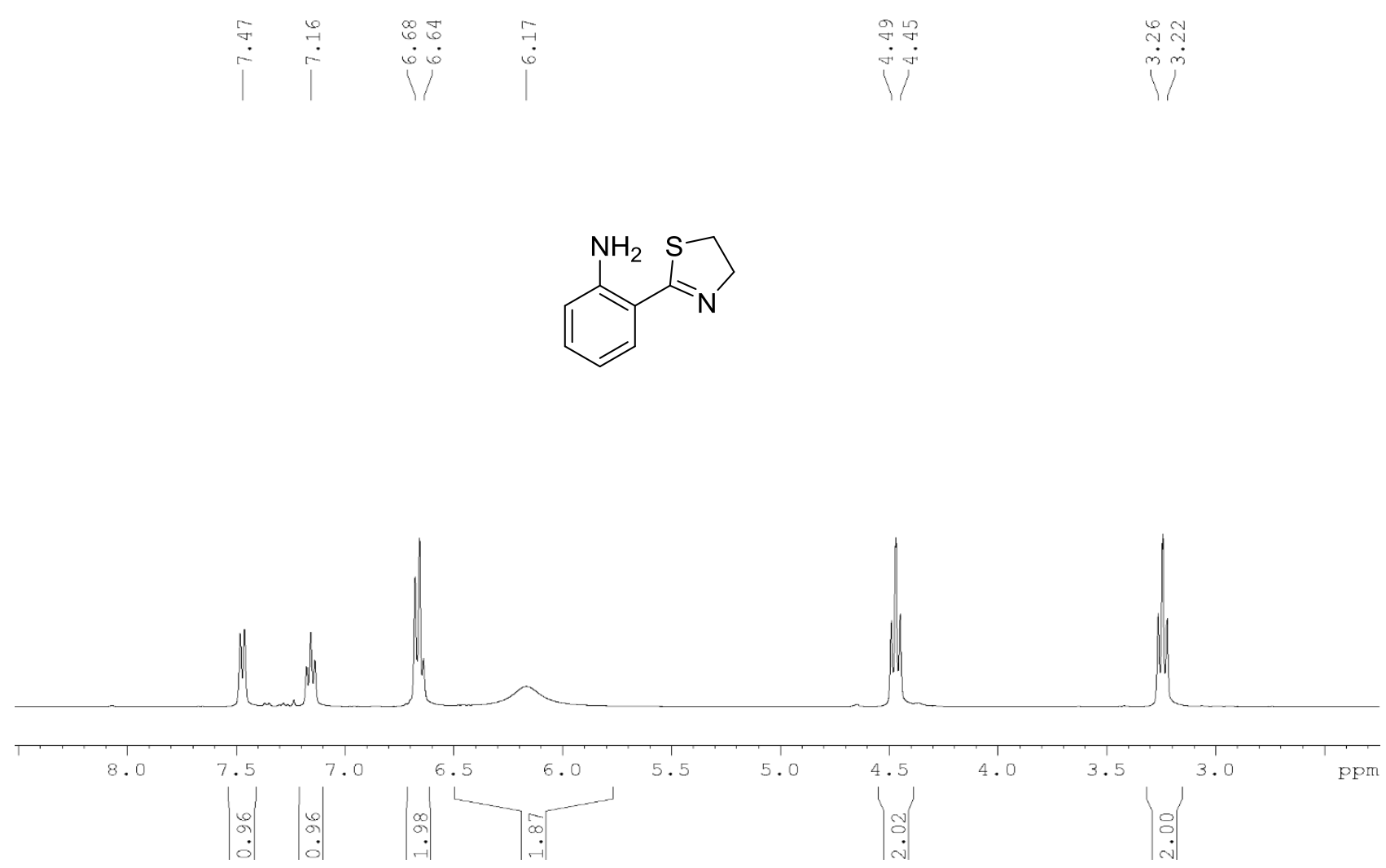

${ }^{13} \mathrm{C}-\left\{{ }^{1} \mathrm{H}\right\}$ NMR (75 MHz, $\left.\mathrm{CDCl}_{3}, 298 \mathrm{~K}\right)$ of 2-(4,5-dihydrothiazol-2-yl)aniline, 4n
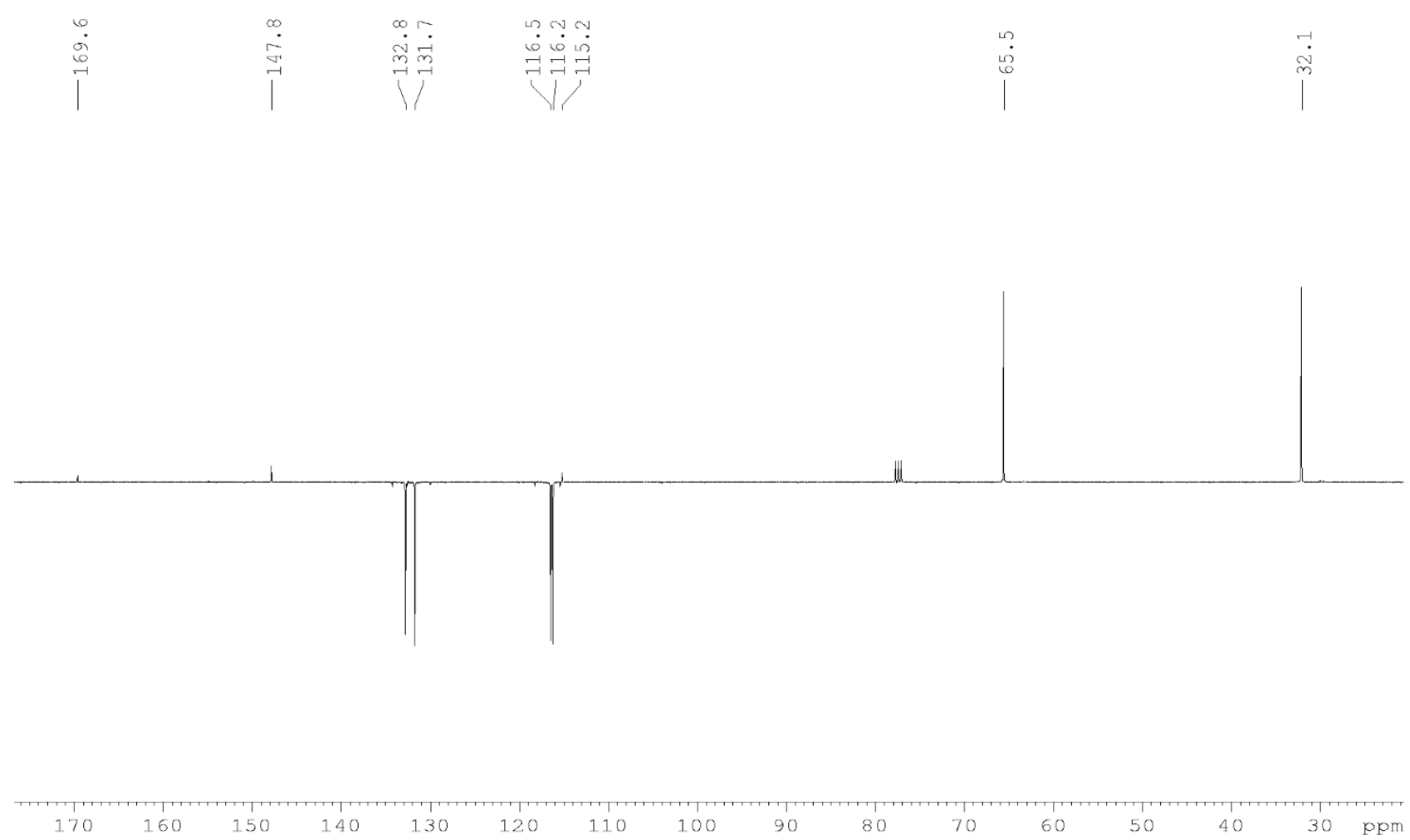
${ }^{1} \mathrm{H}$ NMR (400 MHz, CDCl 3 , 298K, TMS) of 2-(4-chlorobenzyl)-4,5-dihydrothiazole, $4 \mathbf{o}$
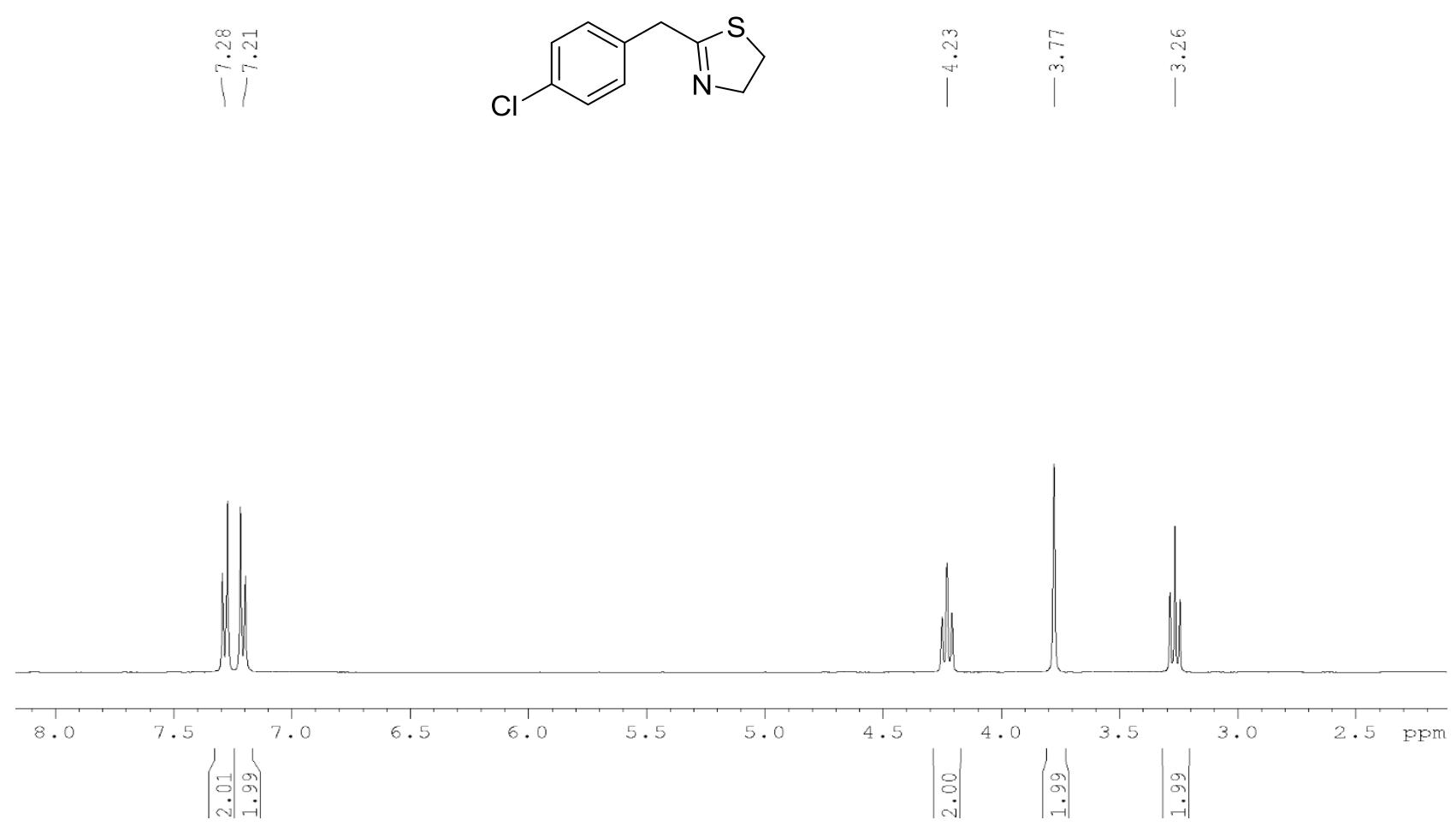

${ }^{13} \mathrm{C}-\left\{{ }^{1} \mathrm{H}\right\}$ NMR (75 MHz, $\left.\mathrm{CDCl}_{3}, 298 \mathrm{~K}\right)$ of 2-(4-chlorobenzyl)-4,5-dihydrothiazole, $4 \mathbf{o}$
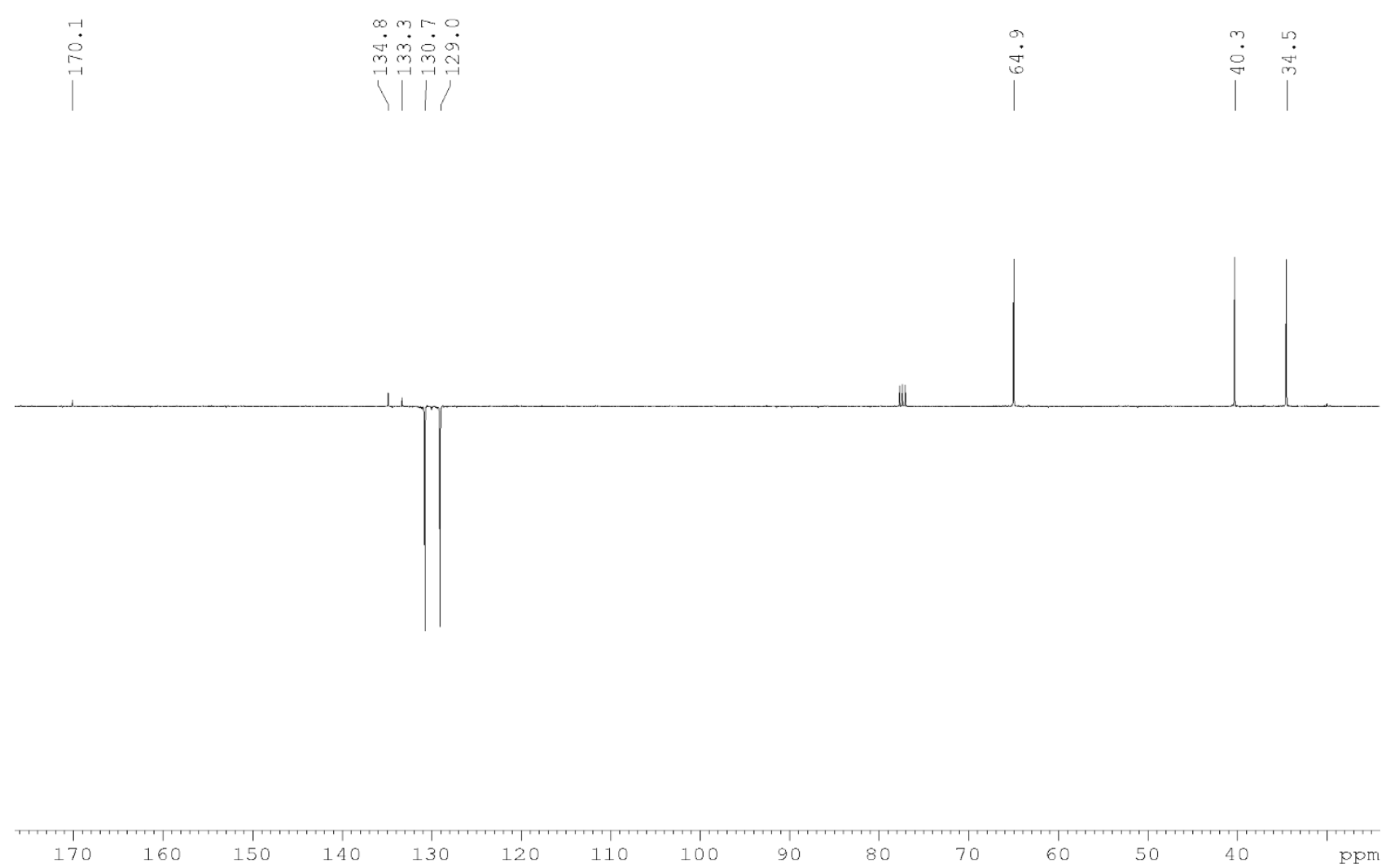
${ }^{1} \mathrm{H}$ NMR (400 MHz, $\mathrm{CDCl}_{3}, 298 \mathrm{~K}$, TMS) of 2-benzyl-4,5-dihydrothiazole, $4 \mathbf{p}$
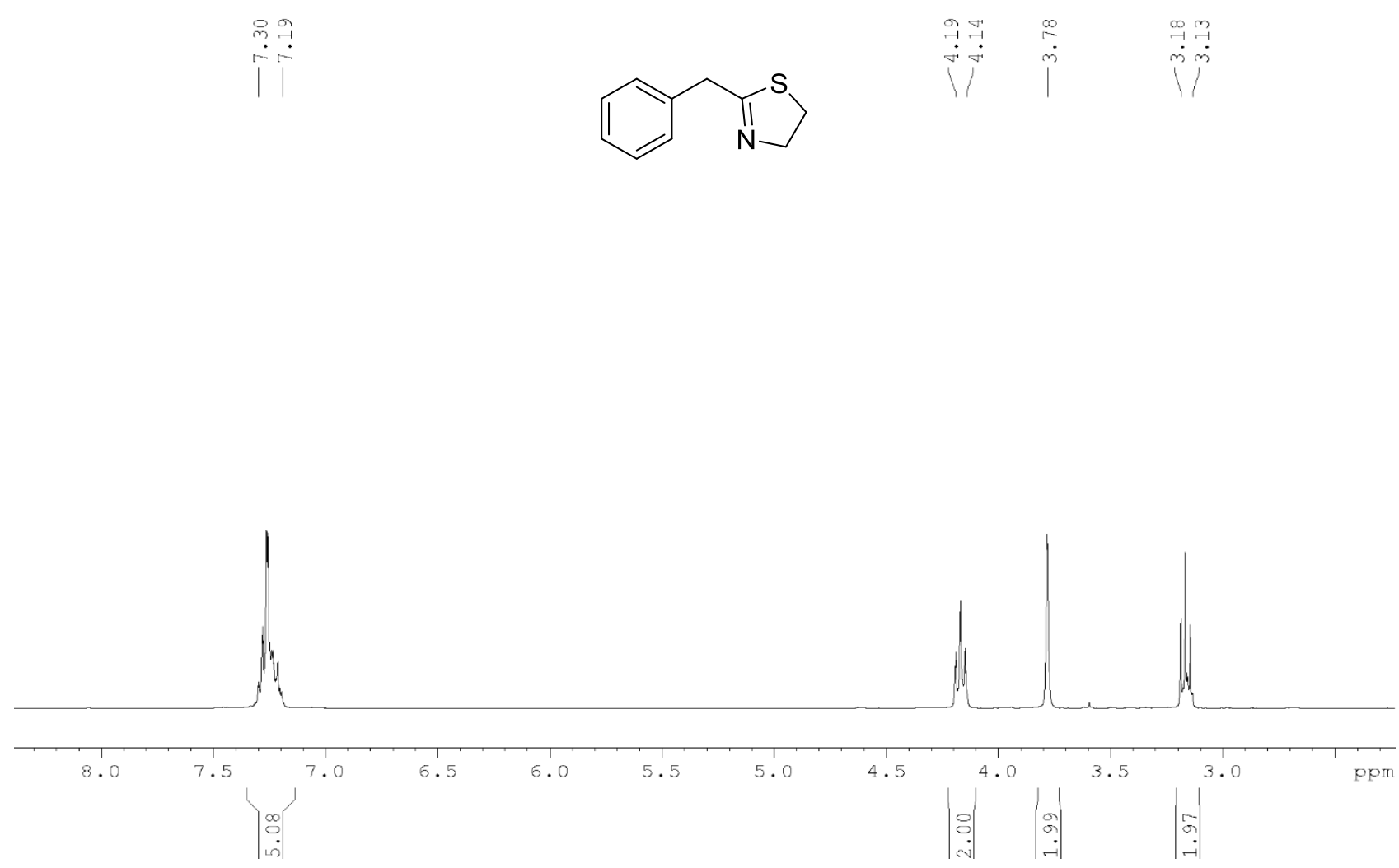

${ }^{13} \mathrm{C}-\left\{{ }^{1} \mathrm{H}\right\}$ NMR $\left(75 \mathrm{MHz}, \mathrm{CDCl}_{3}, 298 \mathrm{~K}\right)$ of 2-benzyl-4,5-dihydrothiazole, $4 \mathbf{p}$
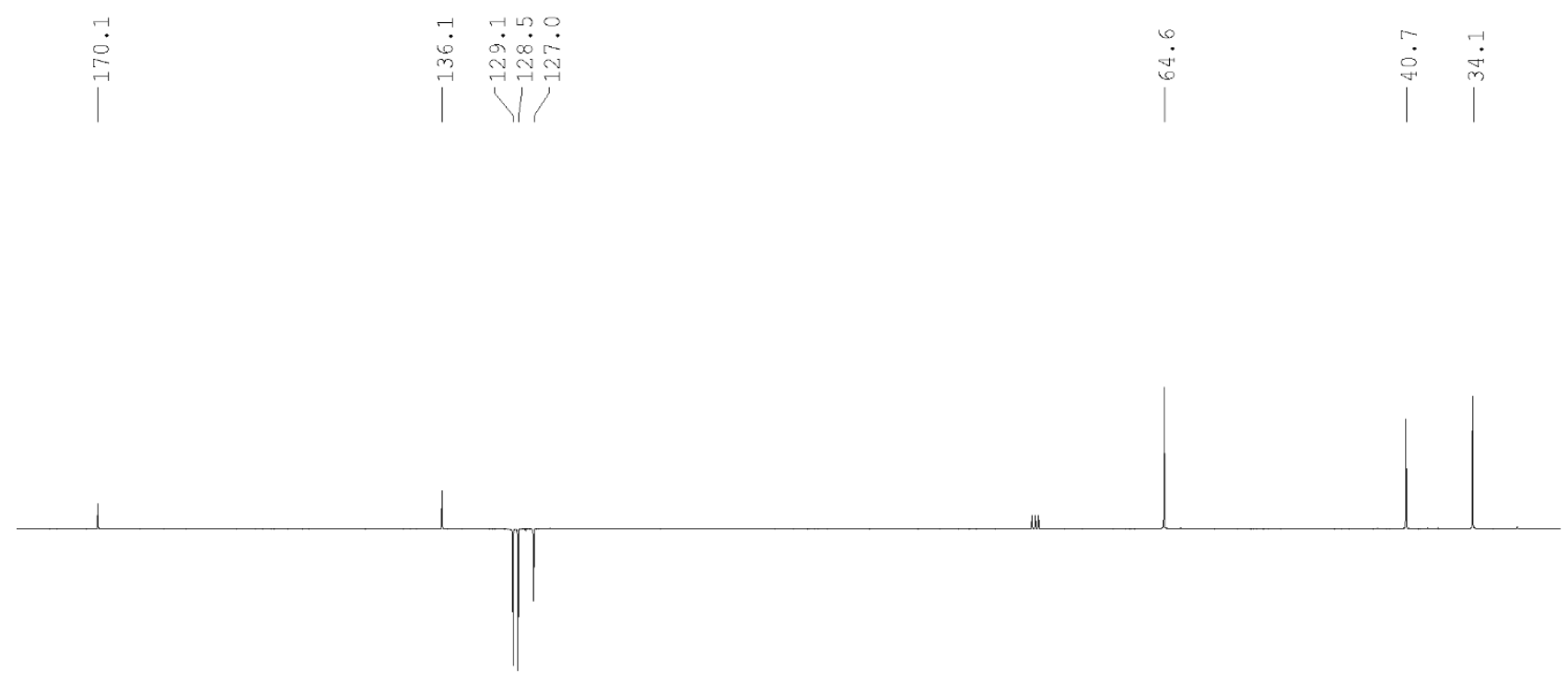

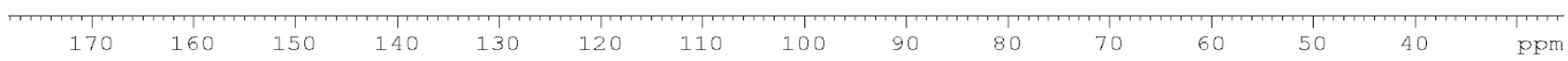


${ }^{1} \mathrm{H}$ NMR (500 MHz, $\mathrm{CDCl}_{3}, 298 \mathrm{~K}$, TMS) of 2-(2-bromobenzyl)-4,5-dihydrothiazole, 4q

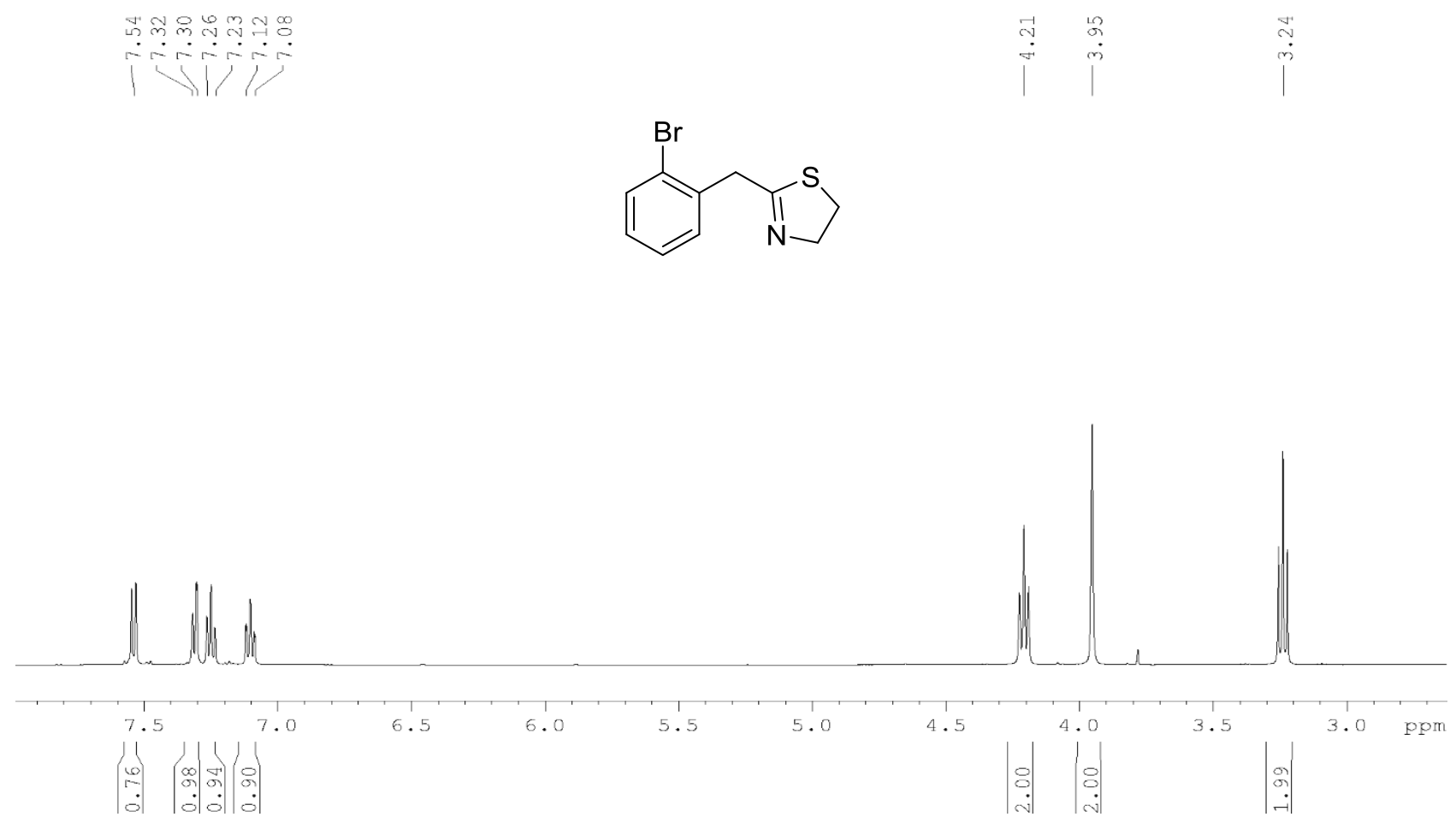

${ }^{13} \mathrm{C}-\left\{{ }^{1} \mathrm{H}\right\} \mathrm{NMR}\left(75 \mathrm{MHz}, \mathrm{CDCl}_{3}, 298 \mathrm{~K}\right)$ of 2-(2-bromobenzyl)-4,5-dihydrothiazole, 4q
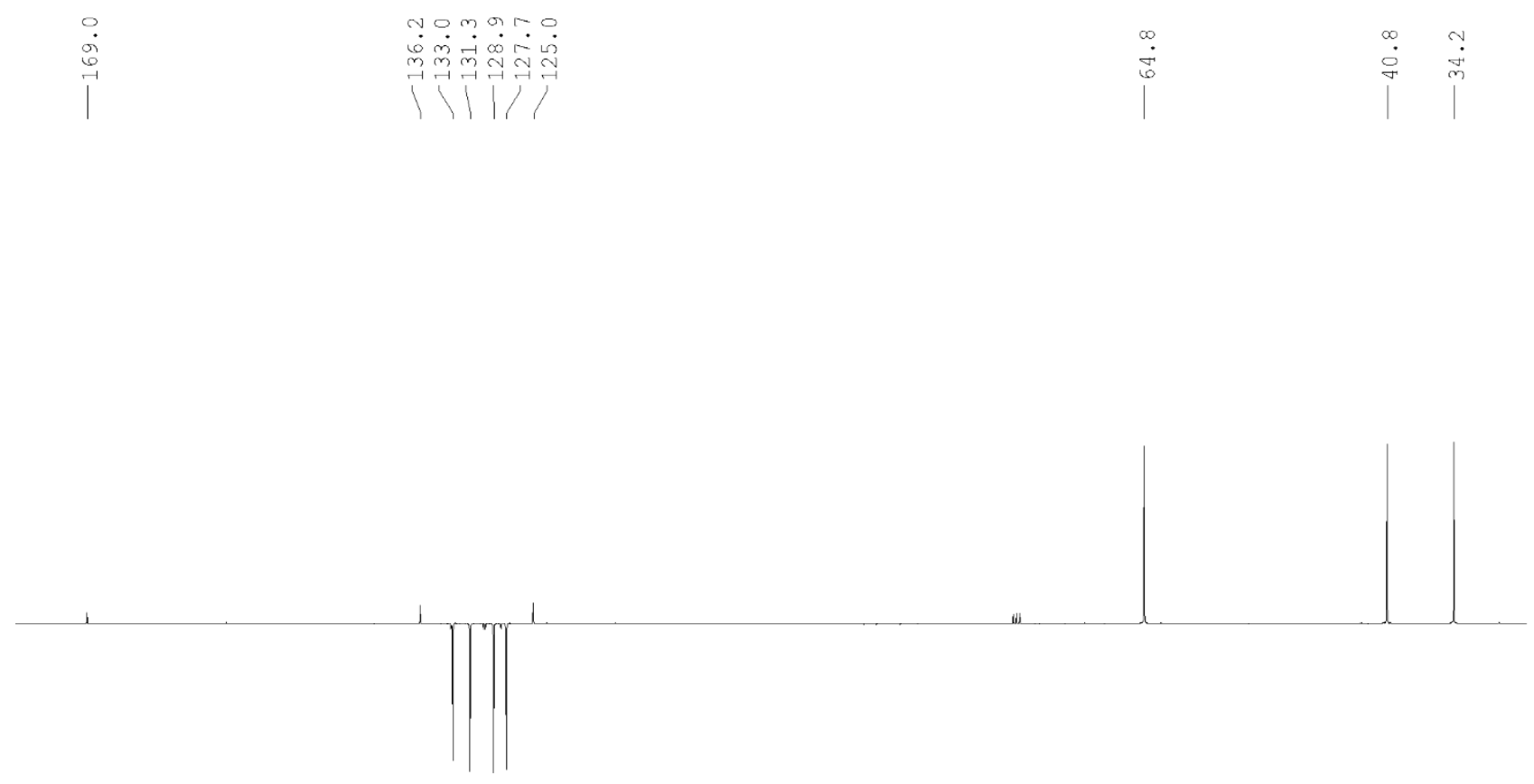
${ }^{1} \mathrm{H}$ NMR (400 MHz, $\mathrm{CDCl}_{3}, 298 \mathrm{~K}$, TMS) of 2-(pyridin-2-yl)-4,5-dihydrothiazole, $4 \mathbf{r}$
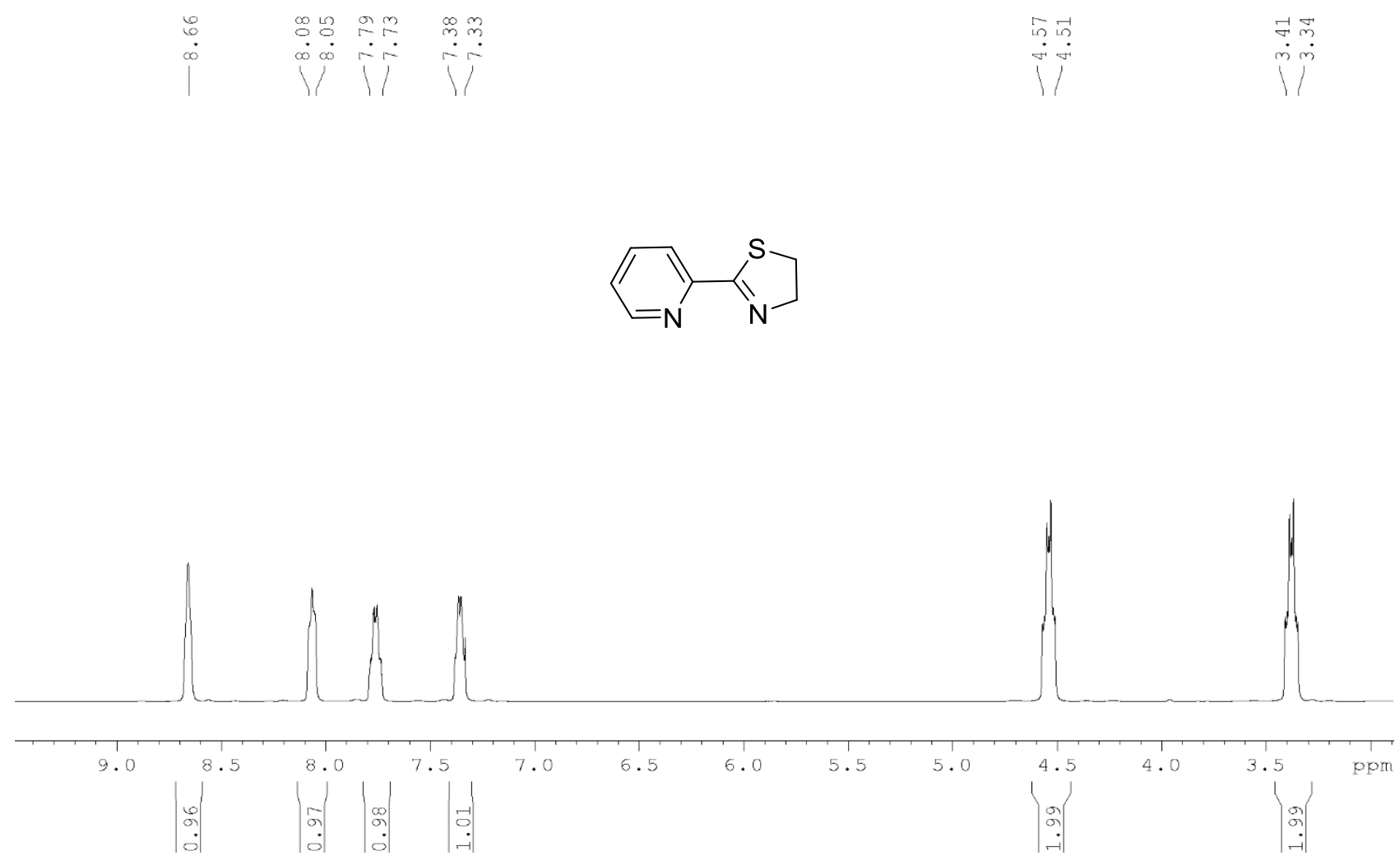

${ }^{13} \mathrm{C}-\left\{{ }^{1} \mathrm{H}\right\}$ NMR $\left(75 \mathrm{MHz}, \mathrm{CDCl}_{3}, 298 \mathrm{~K}\right)$ of 2-(pyridin-2-yl)-4,5-dihydrothiazole, $\mathbf{4 r}$
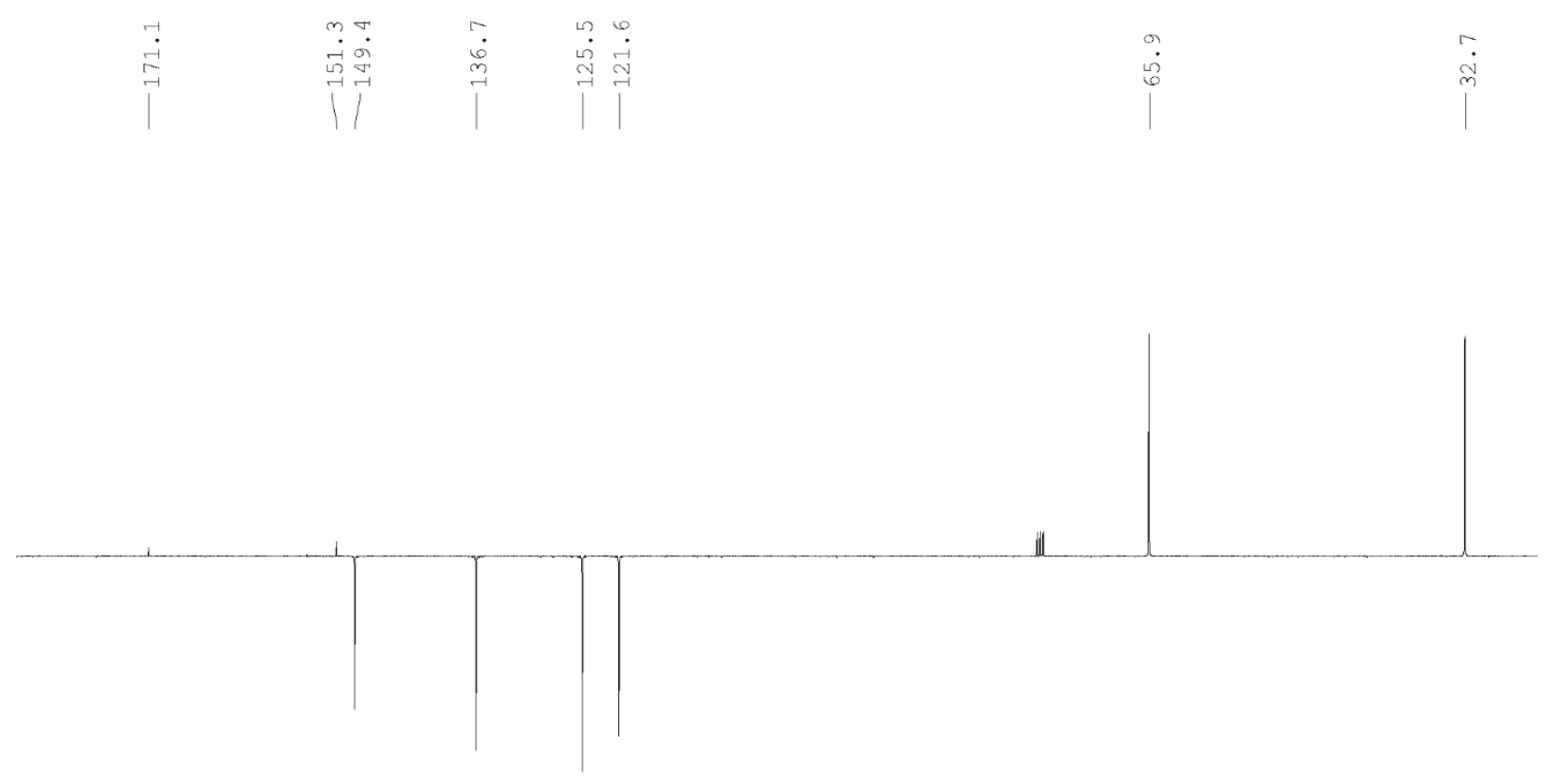

\begin{tabular}{|c|c|c|c|c|c|c|c|c|c|c|c|c|c|c|c|}
\hline 18 & \multicolumn{2}{|c|}{170} & 160 & 150 & 140 & 130 & 120 & 110 & 100 & 90 & 80 & 70 & 60 & 50 & 40 \\
\hline
\end{tabular}


${ }^{1} \mathrm{H}$ NMR (400 MHz, $\mathrm{CDCl}_{3}, 298 \mathrm{~K}$, TMS) of 2-(pyridin-3-yl)-4,5-dihydrothiazole, 4s

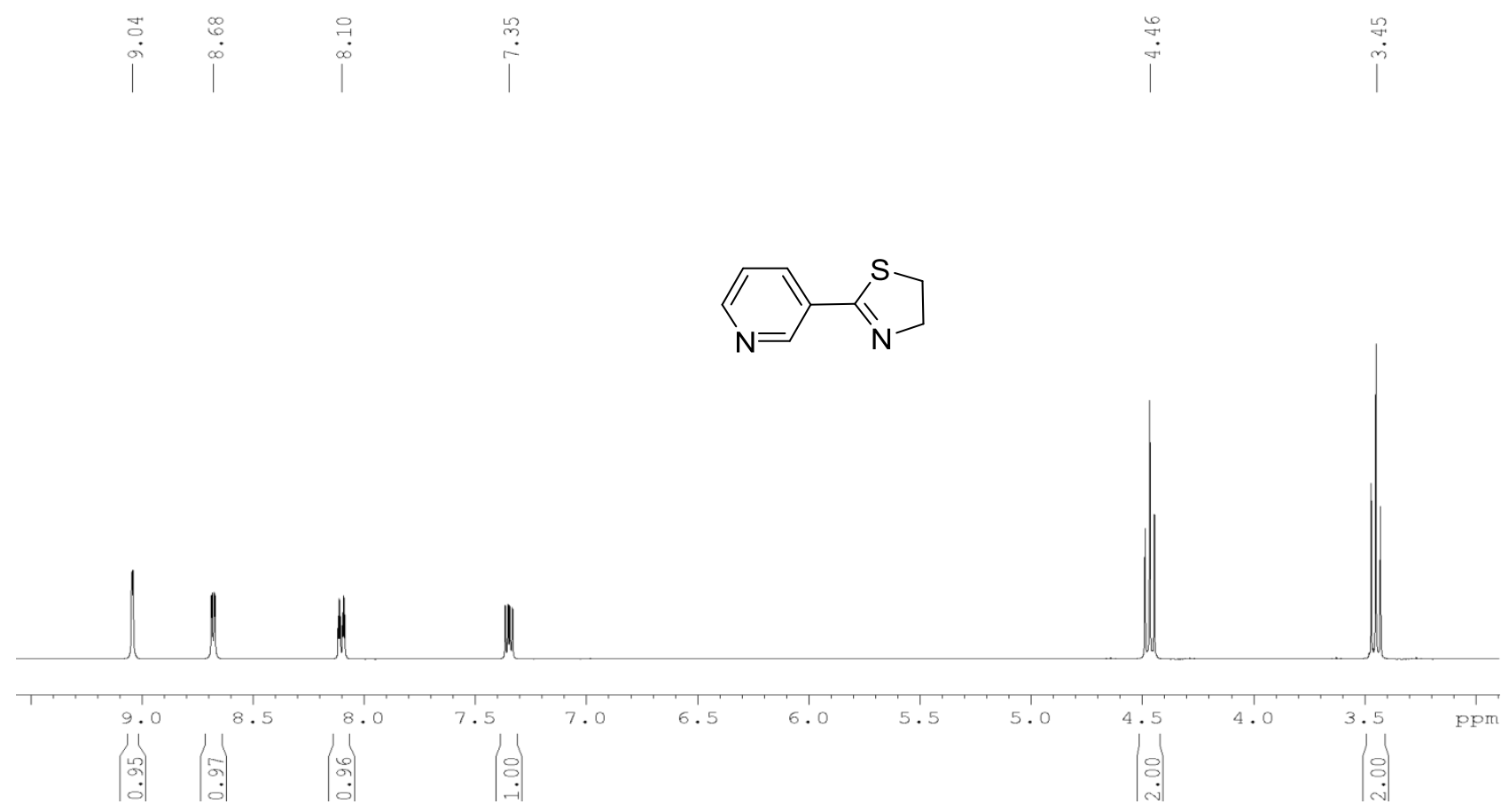

${ }^{13} \mathrm{C}-\left\{{ }^{1} \mathrm{H}\right\}$ NMR (75 MHz, $\left.\mathrm{CDCl}_{3}, 298 \mathrm{~K}\right)$ of 2-(pyridin-3-yl)-4,5-dihydrothiazole, $4 \mathbf{s}$

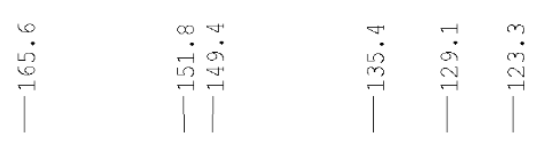

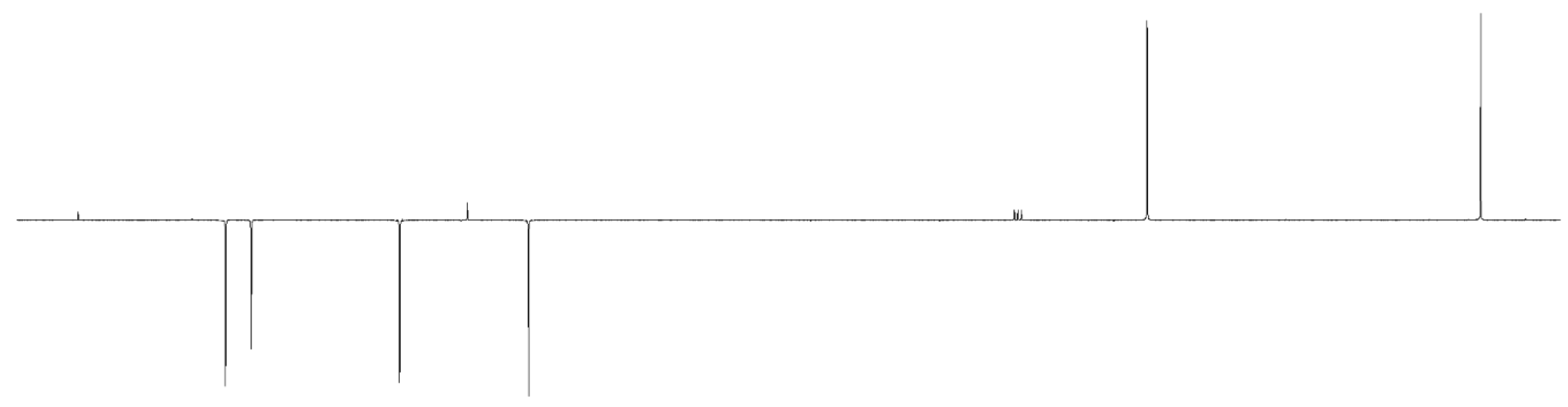


${ }^{1} \mathrm{H}$ NMR (400 MHz, CDCl 3 , 298K, TMS) of 2-(pyridin-4-yl)-4,5-dihydrothiazole, 4t
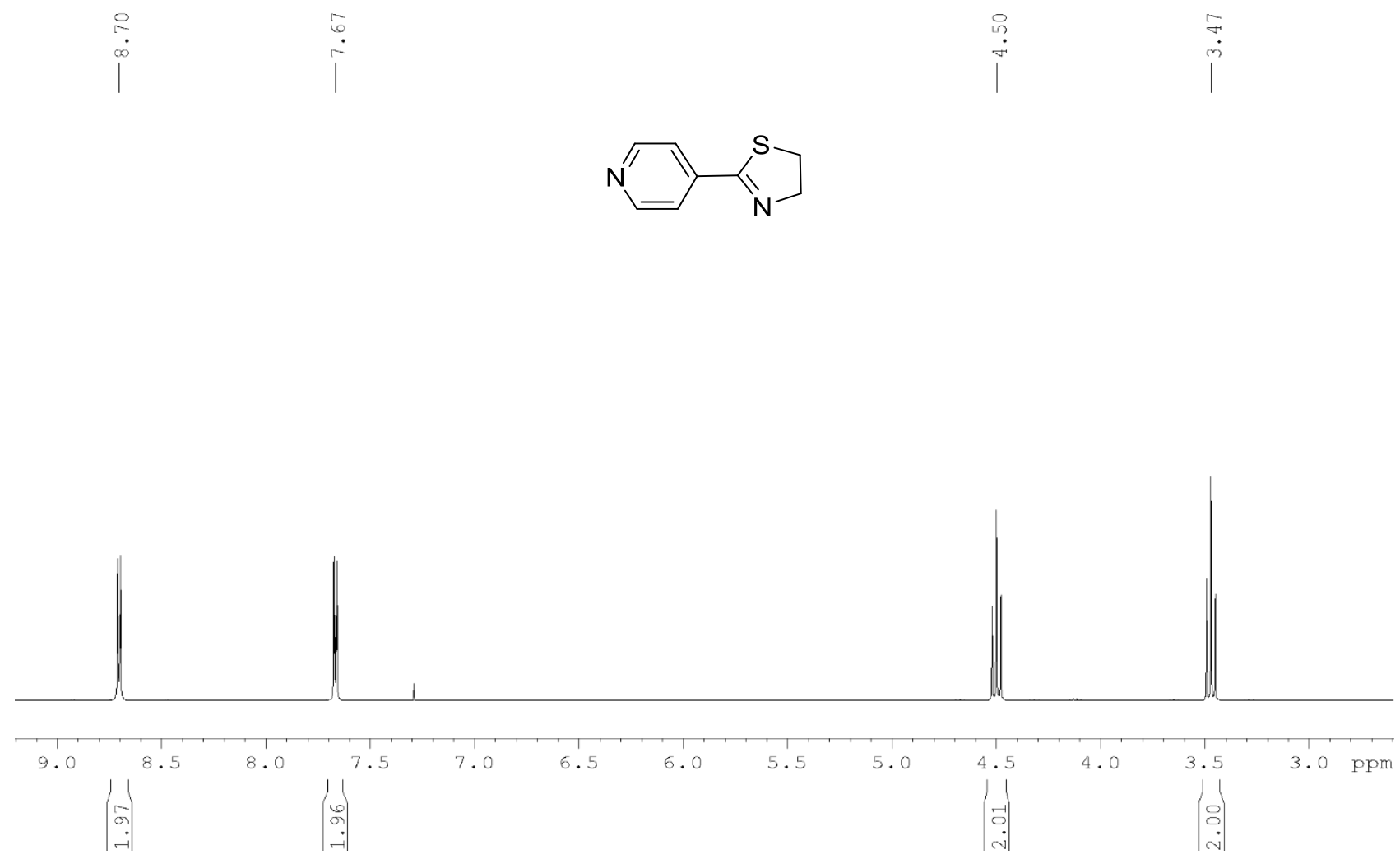

${ }^{13} \mathrm{C}-\left\{{ }^{1} \mathrm{H}\right\}$ NMR (75 MHz, $\left.\mathrm{CDCl}_{3}, 298 \mathrm{~K}\right)$ of 2-(pyridin-4-yl)-4,5-dihydrothiazole, $4 \mathbf{t}$

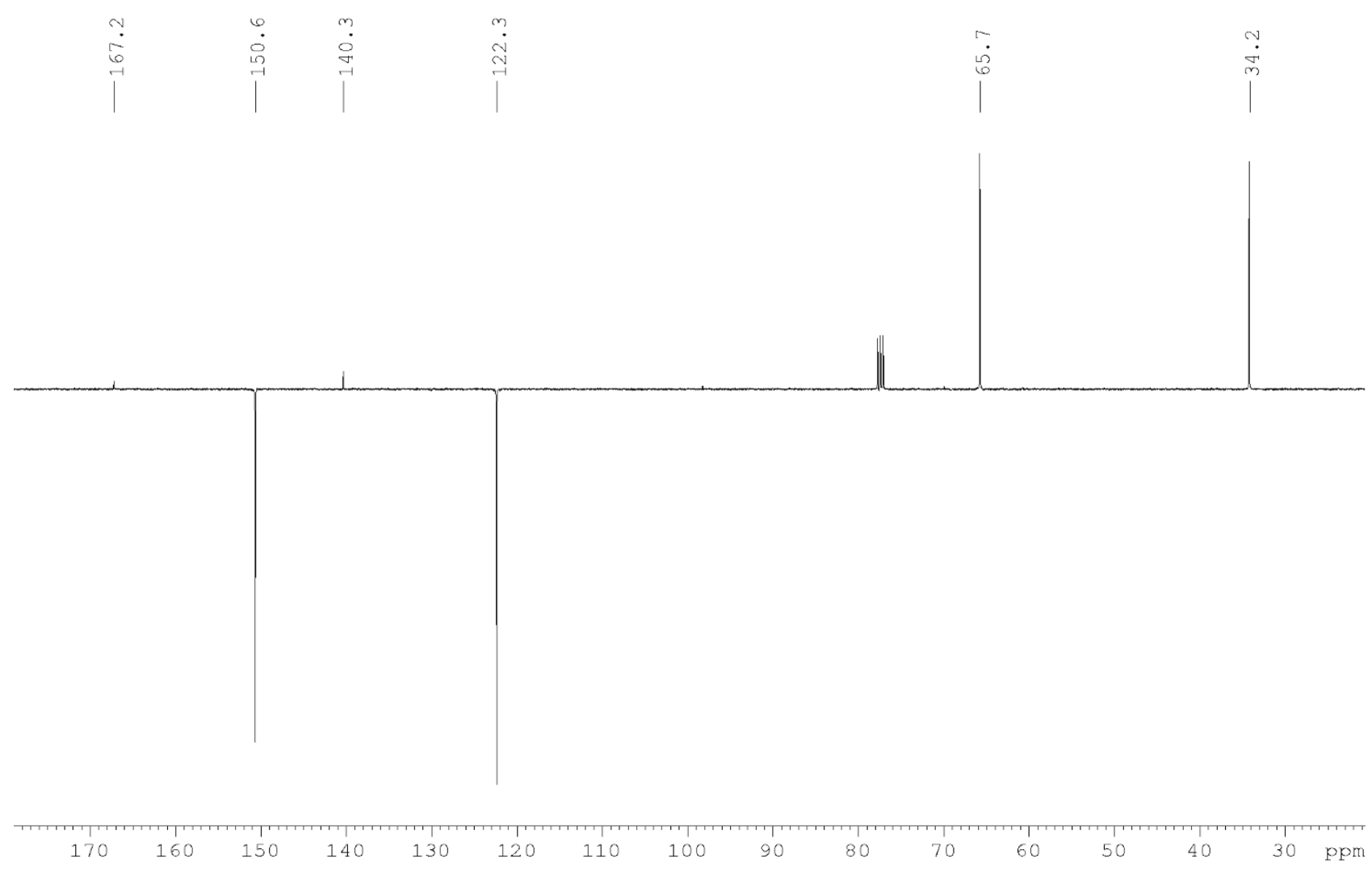


${ }^{1} \mathrm{H}$ NMR (400 MHz, CDCl 3 , 298K, TMS) of 2-(1H-pyrrol-2-yl)-4,5-dihydrothiazole, 4u
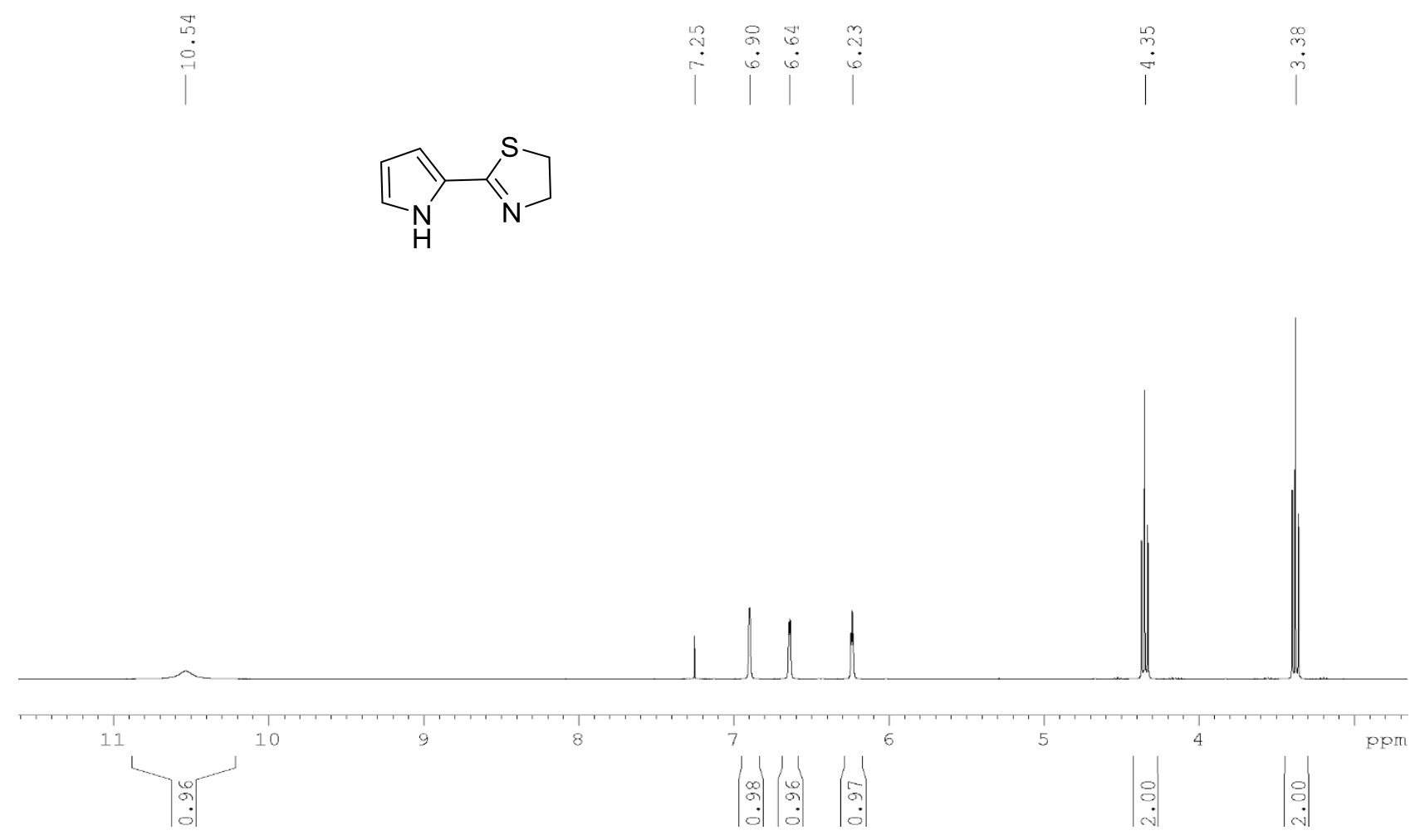

${ }^{13} \mathrm{C}-\left\{{ }^{1} \mathrm{H}\right\}$ NMR (75 MHz, CDCl $\left.3,298 \mathrm{~K}\right)$ of 2-(1H-pyrrol-2-yl)-4,5-dihydrothiazole, $4 \mathbf{u}$
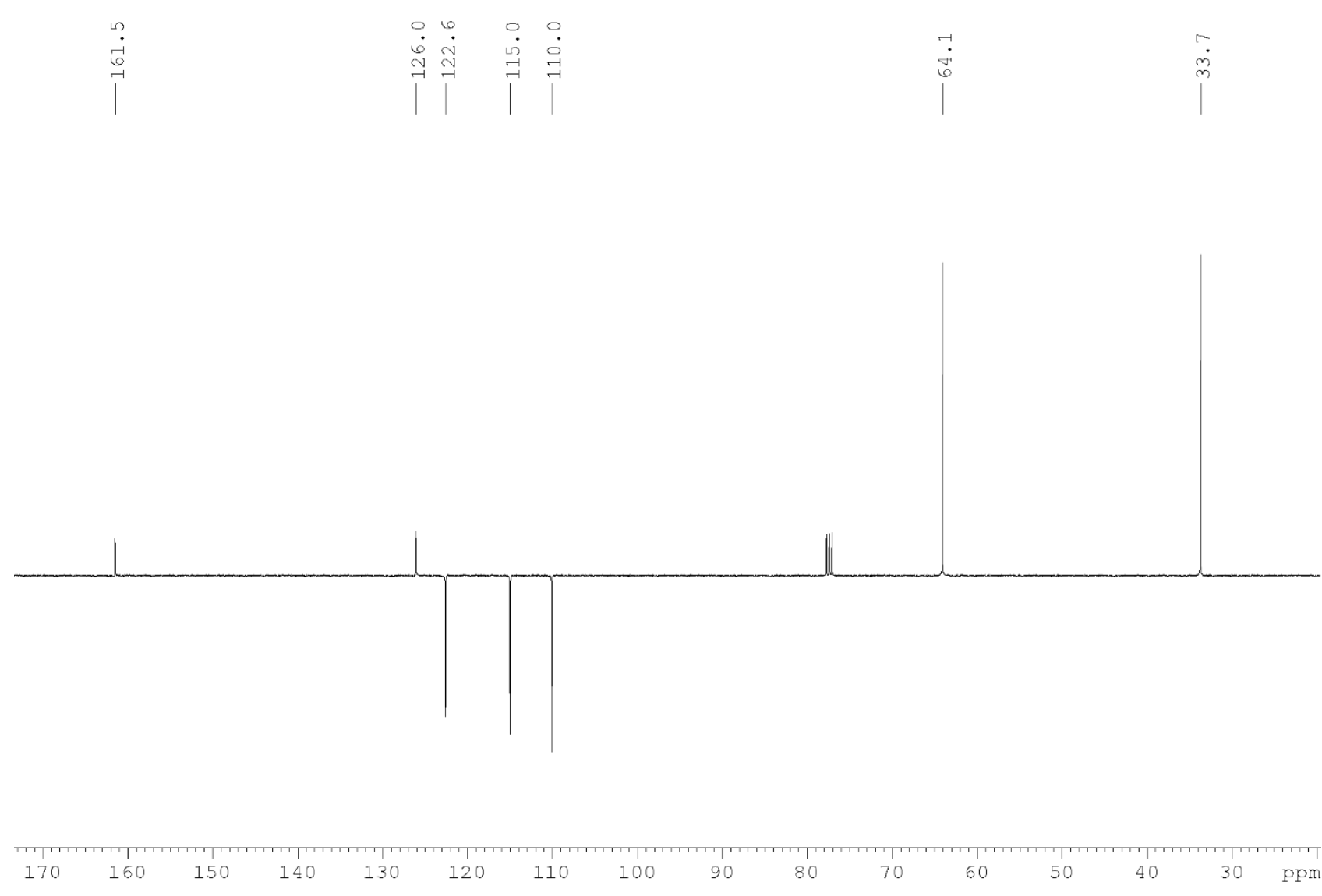
${ }^{1} \mathrm{H}$ NMR (400 MHz, CDCl 3 , 298K, TMS) of 2-(2-furanyl)-4,5-dihydrothiazole, 4v

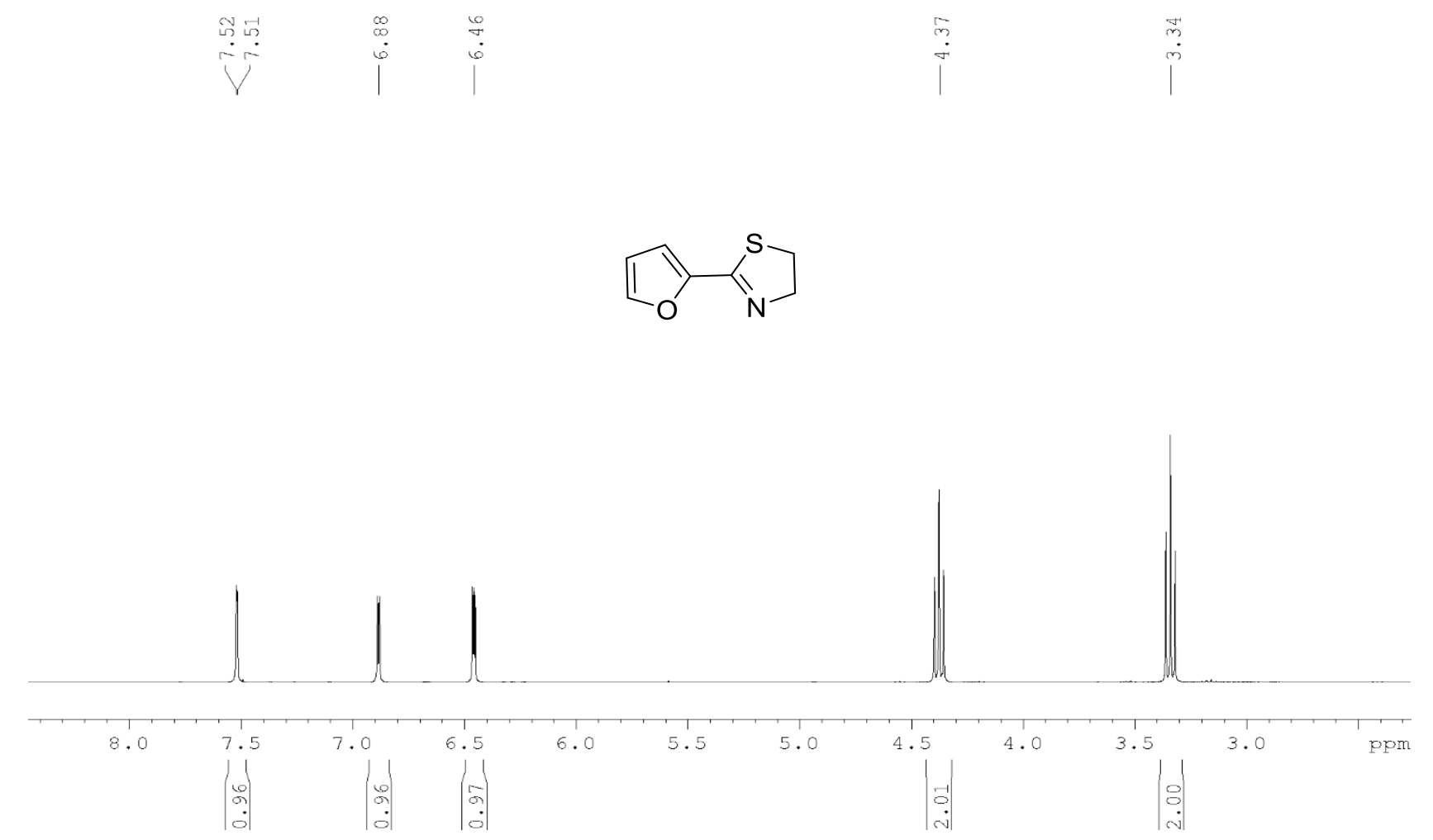

${ }^{13} \mathrm{C}-\left\{{ }^{1} \mathrm{H}\right\}$ NMR (75 MHz, $\mathrm{CDCl}_{3}, 298 \mathrm{~K}$ ) of 2-(2-furanyl)-4,5-dihydrothiazole, $4 \mathbf{v}$

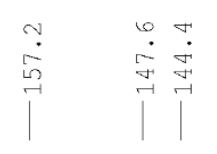

离

$\stackrel{?}{\circ}$

$\stackrel{-}{n}$
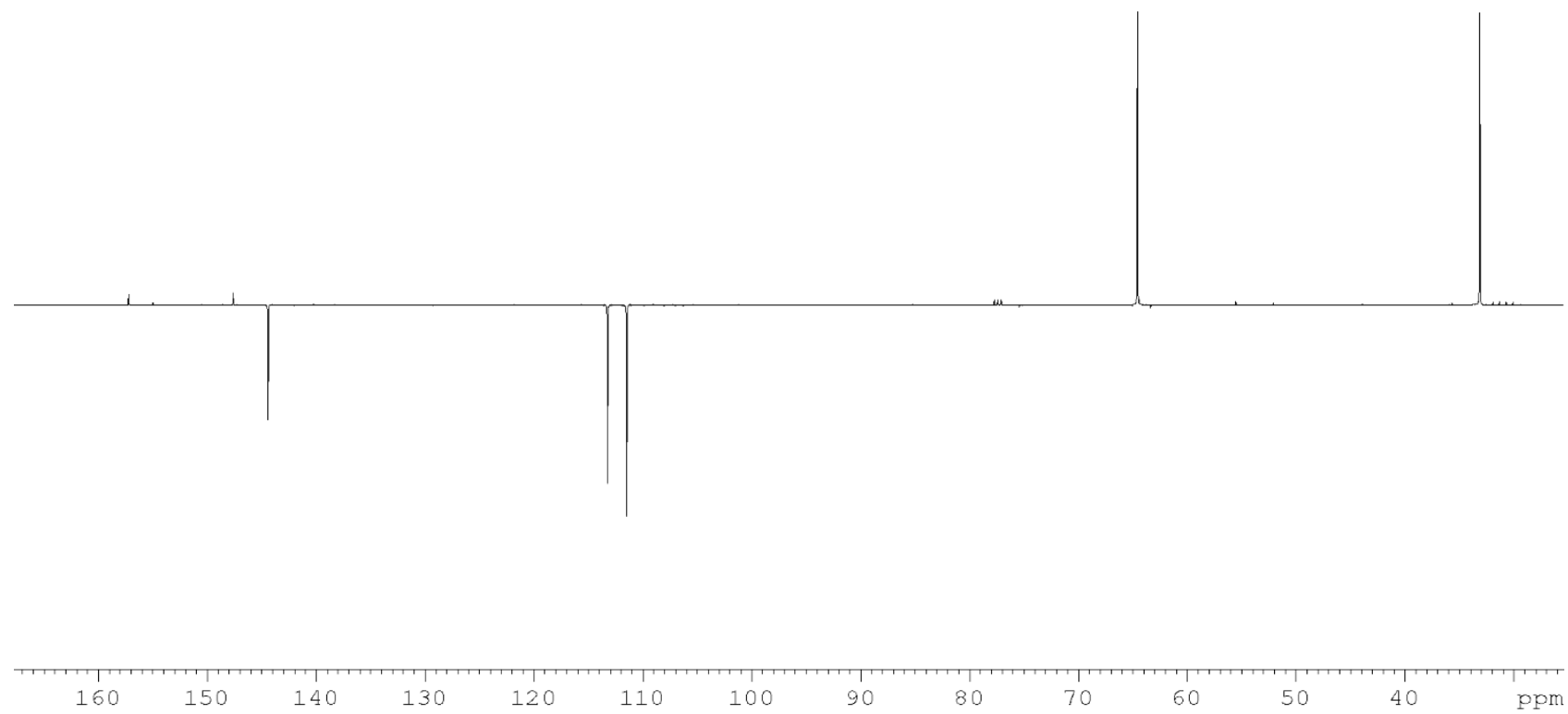
${ }^{1} \mathrm{H}$ NMR (400 MHz, CDCl, $298 \mathrm{~K}$, TMS) of 2-(pent-4-yn-1-yl)-4,5-dihydrothiazole, 4w
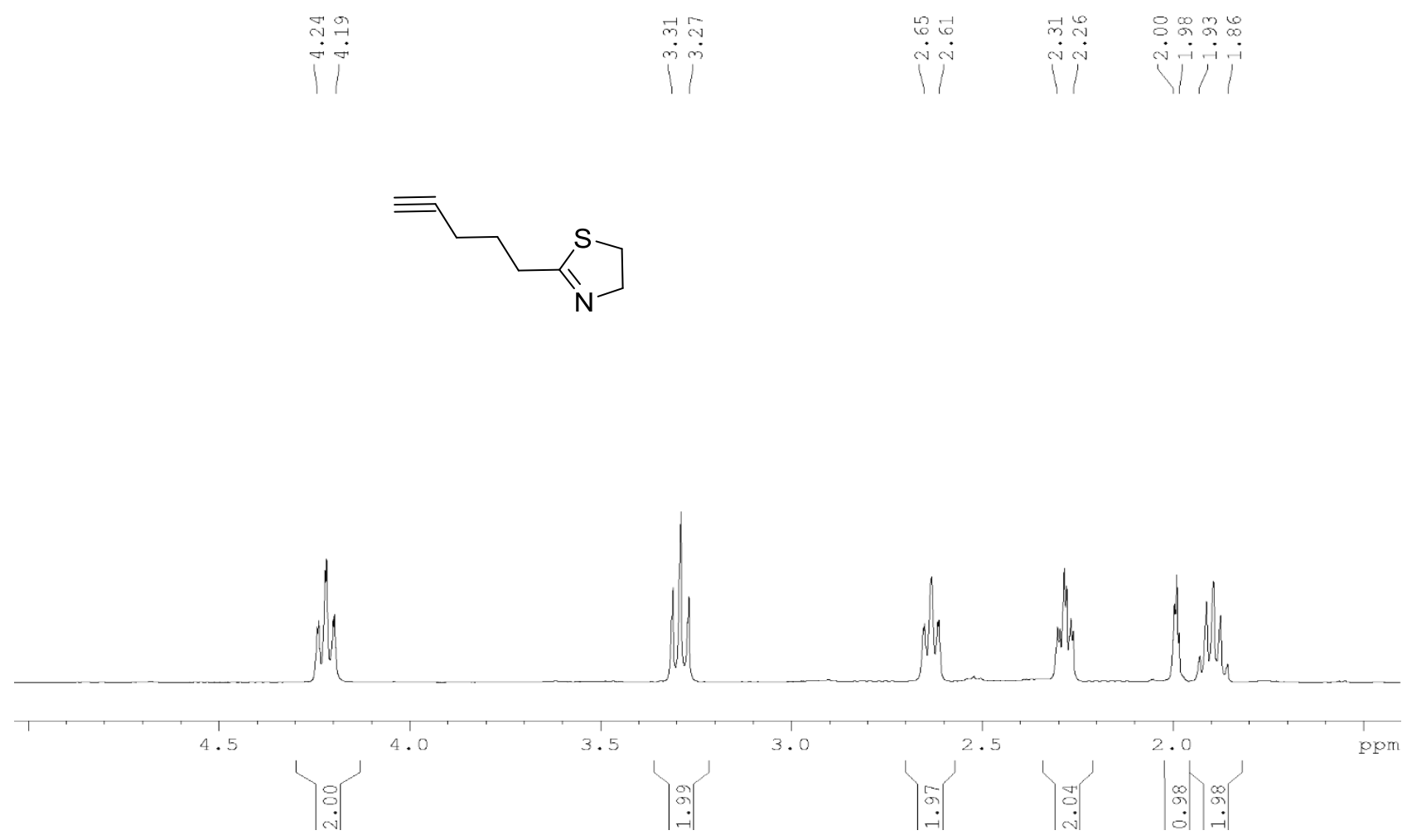

${ }^{13} \mathrm{C}-\left\{{ }^{1} \mathrm{H}\right\}$ NMR (75 MHz, CDCl $\left.3,298 \mathrm{~K}\right)$ of 2-(pent-4-yn-1-yl)-4,5-dihydrothiazole, $4 \mathbf{w}$ 辛

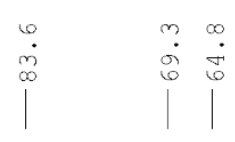

$\begin{array}{lll}0 & m & m \\ \dot{m} \dot{m} & \dot{0} & \dot{\infty} \\ \mid & & \sim\end{array}$

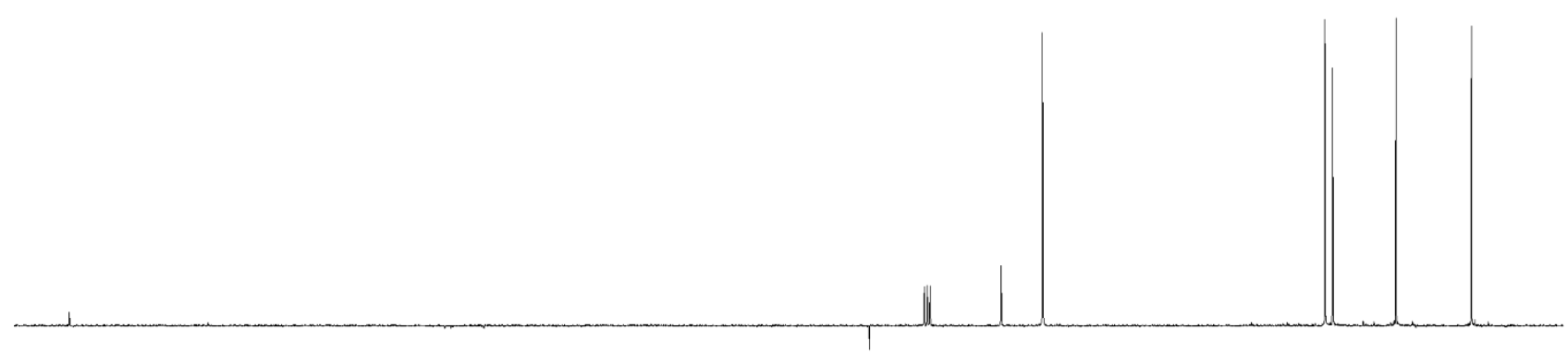

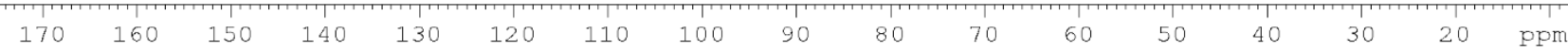


${ }^{1} \mathrm{H}$ NMR (400 MHz, $\mathrm{CDCl}_{3}, 298 \mathrm{~K}$, TMS) of 2-pentyl-4,5-dihydrothiazole, $4 \mathbf{x}$

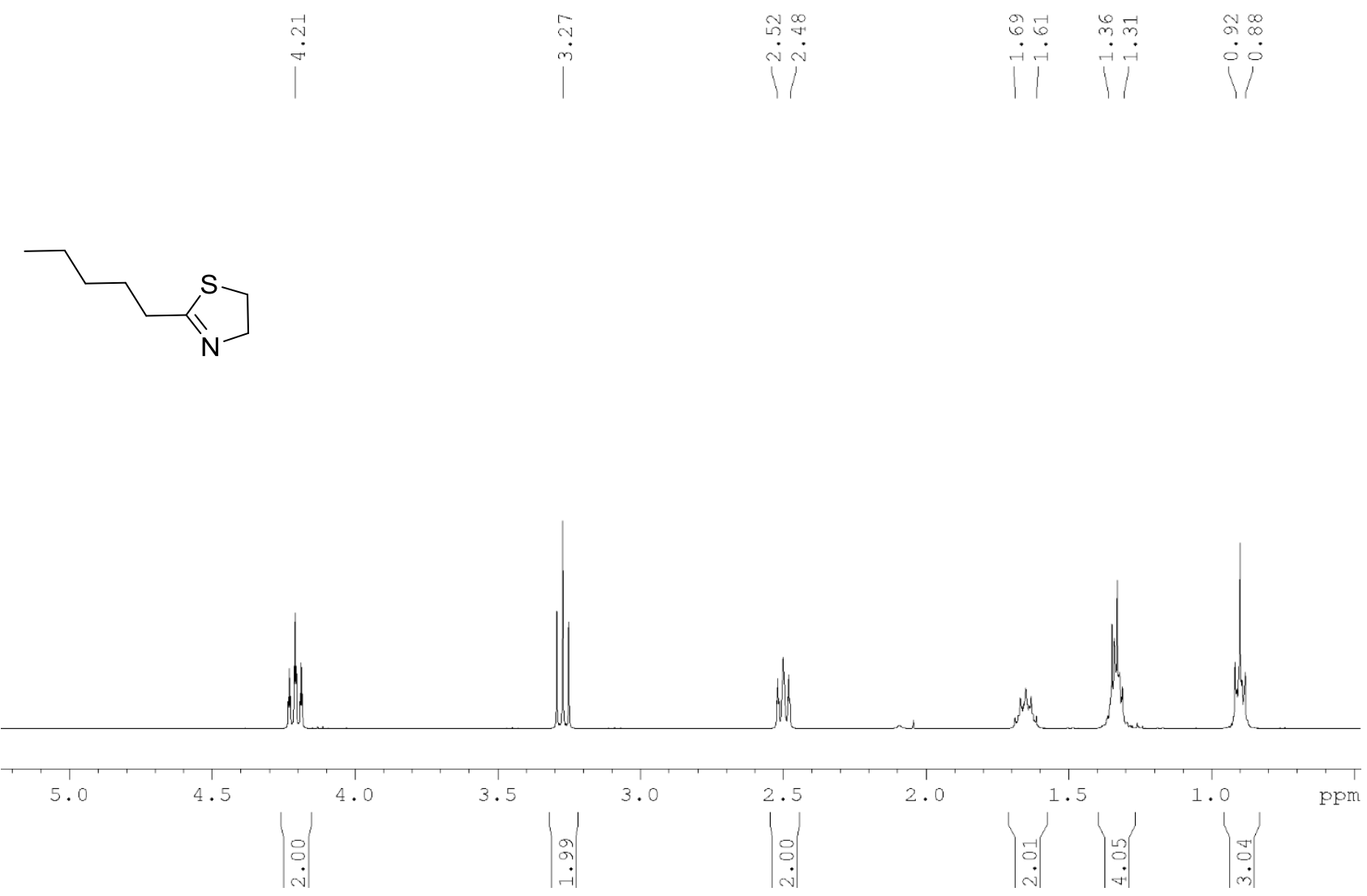

${ }^{13} \mathrm{C}-\left\{{ }^{1} \mathrm{H}\right\} \mathrm{NMR}(75 \mathrm{MHz}, \mathrm{CDCl} 3,298 \mathrm{~K})$ of 2-pentyl-4,5-dihydrothiazole, $4 \mathbf{x}$

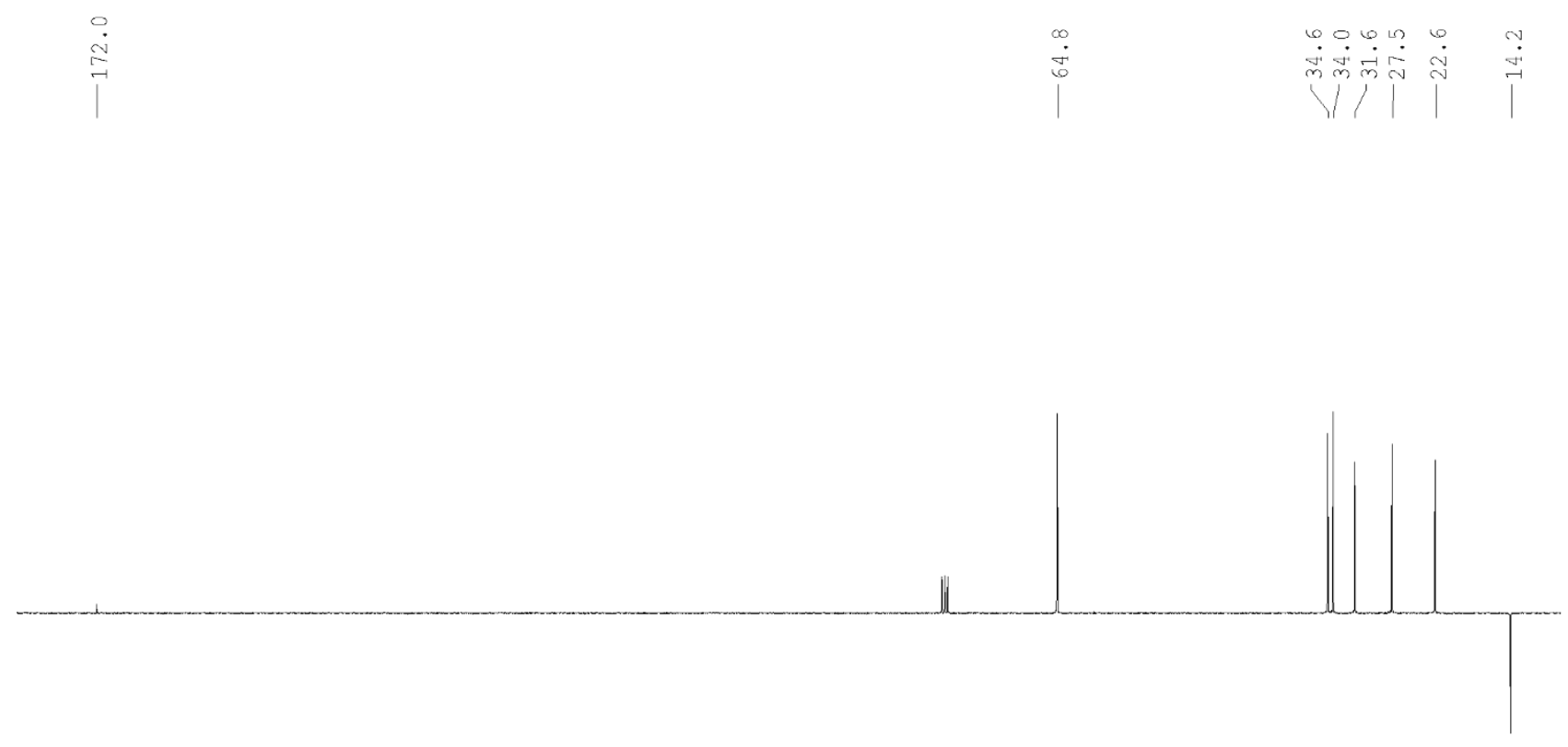

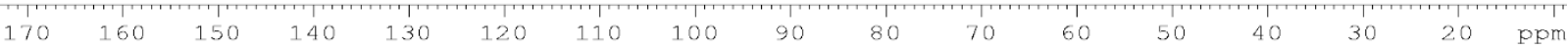


${ }^{1} \mathrm{H}$ NMR (400 MHz, CDCl 3 , 298K, TMS) of 2-cyclohexyl-4,5-dihydrothiazole, 4y
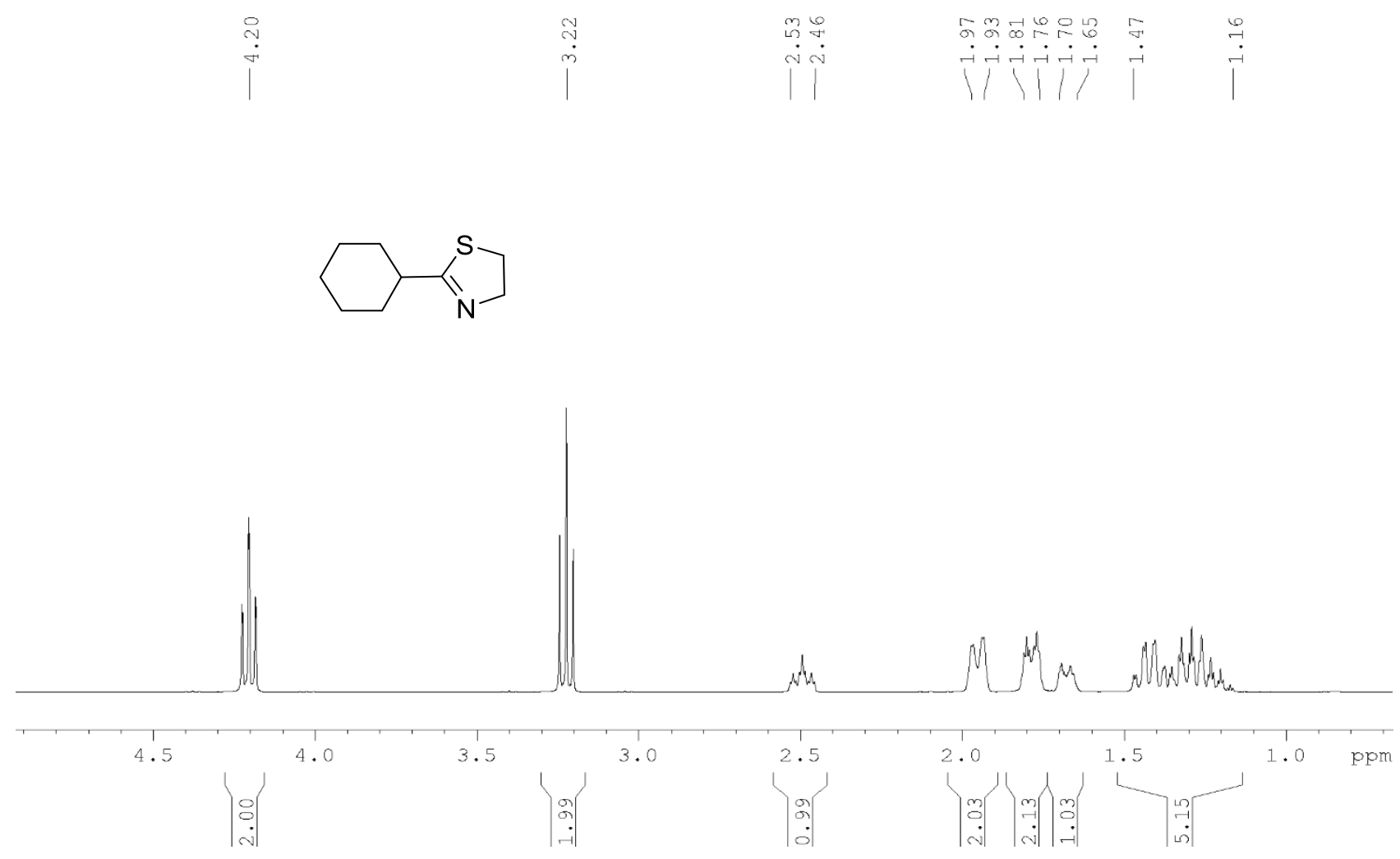

${ }^{13} \mathrm{C}-\left\{{ }^{1} \mathrm{H}\right\}$ NMR $\left(75 \mathrm{MHz}, \mathrm{CDCl}_{3}, 298 \mathrm{~K}\right)$ of 2-cyclohexyl-4,5-dihydrothiazole, $4 \mathbf{y}$
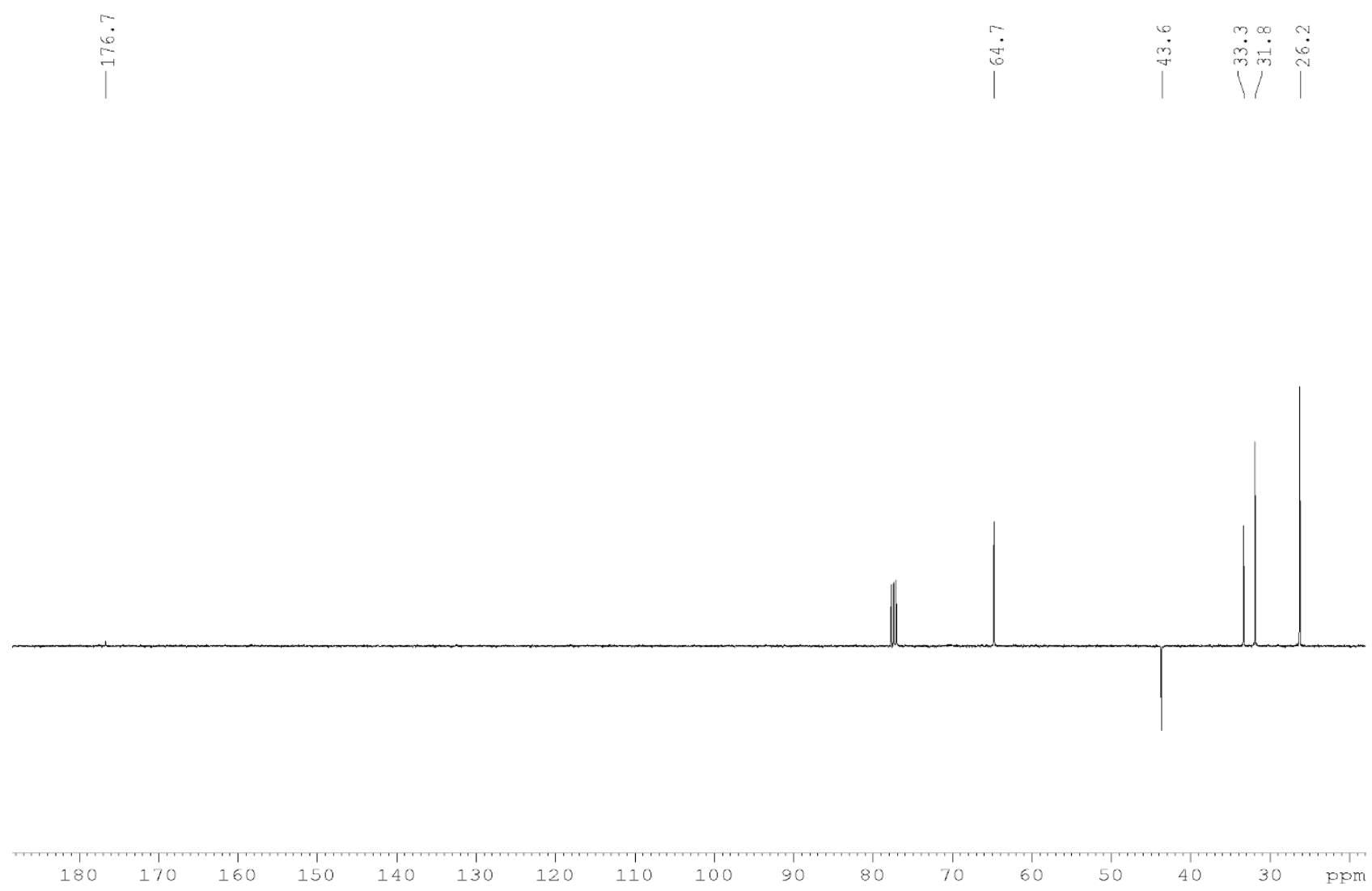


\section{References}

[1] X. Li, B. Zhou, J. Zhang, M. She, S. An, H. Ge, C. Li, B. Yin, J. Li and Z. Shi, Eur. J. Org. Chem. 2012, 8,1626.

[2] L. Wu, E-Journal of Chemistry, 2012, 9, 1035.

[3] U. Pathak, S. Bhattacharyya, V. Dhruwansh, L. K. Pandey, R. Tank and M. V. S. Suryanarayana, Green Chem., $2011,13,1648$

[4] S. H. Babcock and R. Adams, J. Am. Chem. Soc., 1937, 59, 2260.

[5] S. F. Hojati, I. Mohammadpoor-Baltork, B. Maleki, M. Gholizadeh, F. Shafiezadeh and M. Haghdous, Can. J. Chemistry, 2010, 88, 135.

[6] J. A. Seijas, M. P. Vázquez-Tato, J. Crecente-Campo, Tetrahedron, 2008, 64, 9280.

[7] B. George, E. P. Papadopoulos, J. Org. Chem., 1977, 42, 441.

[8] A. I. Meyers and J. L. Durandetta, J. Org. Chem., 1975, 40, 2021. 